Comparative effects of lifestyle intervention, low calorie diet and bariatric surgery on weight loss and arterial stiffness.

Non-randomized clinical trials including treatment seeking morbidly obese patients.

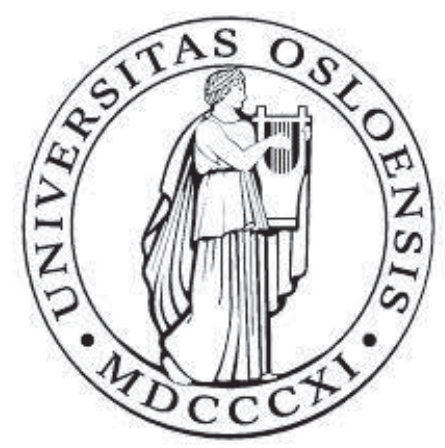

Espen Gjevestad

Clinic for Physical Medicine and Rehabilitation \& the Morbid Obesity Center, Vestfold Hospital Trust, Norway

Faculty of Medicine, University of Oslo 
(C) Espen Gjevestad, 2015

Series of dissertations submitted to the Faculty of Medicine, University of Oslo No. 1917

ISBN 978-82-8264-960-5

All rights reserved. No part of this publication may be reproduced or transmitted, in any form or by any means, without permission.

Cover: Hanne Baadsgaard Utigard.

Printed in Norway: AIT Oslo AS.

Produced in co-operation with Akademika Publishing.

The thesis is produced by Akademika Publishing merely in connection with the thesis defence. Kindly direct all inquiries regarding the thesis to the copyright holder or the unit which grants the doctorate. 


\section{Contents}

$\begin{array}{ll}\text { Acknowledgements } & 7\end{array}$

Abbreviations $\quad 9$

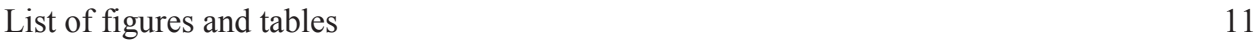

$\begin{array}{ll}\text { List of papers } & 13\end{array}$

$1 \quad$ Introduction and background 14

$\begin{array}{ll}\text { 1.1 What is this thesis about? } & 14\end{array}$

$\begin{array}{ll}1.2 \text { Obesity } & 17\end{array}$

$\begin{array}{ll}\text { 1.2.1 Definition of obesity } & 17\end{array}$

$\begin{array}{ll}\text { 1.2.2 Obesity trends/prevalence } & 19\end{array}$

$\begin{array}{ll}1.3 \text { The burden of obesity } & 20\end{array}$

$\begin{array}{ll}\text { 1.3.1 Aortic arterial stiffness } & 22\end{array}$

$\begin{array}{ll}\text { 1.3.2 Physical inactivity } & 23\end{array}$

1.3.3 Health related quality of life 23

1.4 Obesity treatment strategies $\quad 24$

1.4.1 Guidelines for the treatment of obesity in Norway 25

$\begin{array}{ll}\text { 1.4.2 Physical activity } & 25\end{array}$

$\begin{array}{ll}\text { 1.4.3 Behavioral change } & 26\end{array}$ 
3.5 Sample size

4.1 Paper I 
References

Paper I

Paper II

Paper III 


\section{Acknowledgements}

The work presented in this thesis was carried out between 2006 and 2013 at the Morbid Obesity Center and Clinic for Physical Medicine and Rehabilitation, both at Vestfold Hospital Trust. The same institutions have founded this work.

Although the work with this thesis has sometimes been lonely, frustrating, demanding and at times exhausting, it has been a learning experience which has inspired my academic curiosity.

Although a $\mathrm{PhD}$ is very much a personal degree, there are many people which have contributed to the research process and should be acknowledged.

First of all, my sincere gratitude goes to my main supervisor Professor Jøran Hjelmesæth. He is a highly skilled and patient supervisor, and he has tirelessly guided, supported and encouraged me throughout this work.

I would also like to thank my co-supervisors Professor Sverre Mæhlum for his scientific contribution to this work, and Dr. Liv Marit Smedstad for her scientific contribution, ensuring my founding as a $\mathrm{PhD}$-student, and in the initiation of the intensive lifestyle intervention at Clinic Physical Medicine and Rehabilitation.

The contribution of both former and current colleagues at both the Clinic for Physical Medicine and Rehabilitation, and the Morbid Obesity Centre has been essential in this work. My sincere thanks goes to Njord Nordstrand, Tor Ivar Karlsen, Rune Sandbu, Jo Røislien, Jens Hertel, Line Kristin Johnson and last but not least Erling Saltvedt as invaluable research fellows and co-authors, Linda Mathisen and Kari Bjørn Olsen for organizing patient logistics, Berit Mossing Bjørkås for arranging the biomedical testing av registration, Heidi More Fon for supervising and organizing diets and performing anthropometric measures, and to Matthew McGee for the proofreading of my articles and thesis. 
I would like to express my gratitude to my "team" at the Clinic for Physical Medicine and Rehabilitation, especially Marit Løset Eriksen, Marita Hole Andersen, Mari Sagaas, Jan Hovde, Lise Elgesem Vang, Marit Aske, Torill Kvaløy, Simen Haugen, Sturla Rising, and my highly skilled, crazy colleague, "roommate" and friend Jarle Berge.

Finally, my deepest appreciation goes to my dear wife Christel for being there and trying her best to share and understand my scientific ups and downs, and to my three cornerstones in life; Vilde, Noah and Thale for all the laughter and tears. I love you all!

Stavern, September 2014

Espen Gjevestad 


\section{Abbrevations}

ANCOVA

BIA

BMI

BMR

CFMR

CI

CONOR

CVD

DEXA

ES

GP

GBS

$\mathrm{HbA1C}$

HDL

HOMA-IR

HRQL

ILI

IPAQ-L

LCD

LDL

MAP

MET

MI

MLI

MOC

OWLQOL-17

PWV

${ }_{\text {cf }} \mathrm{PWV}$
Analyses of Covariance

Bioimpedance Analysis

Body Mass Index

Basal Metabolic Rate

Clinic of Physical Medicine and Rehabilitation

Confidence Interval

Cohort of Norway

Cardiovascular Disease

Dual X-ray absorptiometry

Effect Size

General Practitioner

Roux-en-Y Gastric Bypass Surgery

Glycated Hemoglobin

High-Density Lipoprotein Cholesterol

Model Assessment Insulin Resistance

Health Related Quality of Life

Intensive Lifestyle Intervention

Long International Physical Activity Questionnaire

Low Calorie Diet

Low-Density Lipoprotein Cholesterol

Mean Arterial Pressure

Metabolic Equivalent of Task

Multiple Imputation

Moderate Lifestyle Intervention

The Morbid Obesity Center

Obesity and Weight-Loss Quality Of Life

Pulse Wave Velocity

Carotid to femoral Pulse Wave Velocity 
RAAS

RCT

RHA

SD

SF-12

SF-36

$\mathrm{VO}_{2 \max }$

WC

WHO
Renin-angiotensin-aldosterone system

Randomized Controlled Trial

Regional Health Authority

Standard Deviation

12-Item Short Form Health Survey

36-Item Short Form Health Survey

Maximal oxygen consumption

Waist Circumference

World Health Organization 


\section{List of figures and tables}

Figure 1 Flow chart of treatments at the Morbid Obesity Center

Figure 2 Proportion of Norwegian women and men aged 40-44 years with BMI $\geq 30 \mathrm{~kg} / \mathrm{m}^{2}$ from 1965 to 2000

Figure 3 The risk transition

Figure 4 Illustration of a Roux-en-Y gastric bypass

Figure 5 Chart of interventions in paper I

Figure 6 Chart of interventions in paper II and III

Figure 7 Time schedule for the first year of the intensive lifestyle intervention

Figure $8 \quad$ Flow chart of participants in paper I

Figure 9 Mean body weight change after 3 and 12 months of intensive lifestyle intervention or moderate lifestyle intervention

Figure 10 Percentage of all included patients who achieved weight loss $\geq 5 \%$, $\geq 10 \%$, and $\geq 20 \%$ after one year intensive lifestyle intervention or moderate lifestyle intervention

Figure 11 Flow chart of participants in paper II and III

Figure 12 Changes in ${ }_{\mathrm{cf}} \mathrm{PWV}$ after 7 weeks of follow-up

Figure 13 Short- and long-term mean adjusted changes in aortic carotid femoral pulse wave velocity after intensive lifestyle intervention or Roux-en-Y gastric bypass surgery

Figure 14 Scatterplots of the relationship between changes in the explanatory variables body weight, BMI and waist circumference, and changes in the effect variable ${ }_{\mathrm{cf}} \mathrm{PWV}$ within each treatment group

Table 1 The World Health Organisation's (WHO) classification of obesity

Table 2 Relative risk of health problems associated with obesity 
Table 3 Self-reported physical activity at 59 weeks follow up in morbidly

obese patients which completed the interventions

Table 4 Study design and the main limitations and strengths of the paper I, II, and III

Table 5 Linear multiple regression analysis using 7-weeks change in ${ }_{c f} \mathrm{PWV}$ as dependent variable. 


\section{List of papers}

\section{Paper I}

Gjevestad E, Karlsen TI, Røislien J, Mæhlum S, Hjelmesæth J: The effectiveness of secondary and tertiary care lifestyle intervention in morbidly obese patients: a 1-year nonrandomized controlled pragmatic clinical trial. Clinical Obesity, 2013; 3: 39-50.

\section{Paper II}

Nordstrand N, Gjevestad E, Hertel JK, Johnson LK, Saltvedt E, Røislien J, Hjelmesæth J.

Arterial Stiffness, Lifestyle Intervention and a Low-Calorie Diet in Morbidly Obese Patients A Nonrandomized Clinical Trial. Obesity, 2013; 21:690-7.

\section{Paper III}

Gjevestad E, Hjelmesæth J, Sandbu R, Nordstrand N. Effects of Intensive Lifestyle Intervention and Gastric Bypass on aortic stiffness: A 1-Year Nonrandomized Clinical Study. Obesity, 2014; doi:10.1002/oby.20880. 


\section{Chapter 1. Introduction and background}

\subsection{What is this thesis about?}

In 2004, the Norwegian Patients Rights Act was changed to offer morbidly obese persons a formal right to treatment within the specialist health care system ${ }^{1}$. The Ministry of Health and Care Services instructed the five Regional Health Authorities (RHA) to provide morbidly obese patients with an appropriate treatment choice: either conservative or surgical. An interregional working group consisting of surgeons, administrators from the RHA's and internists from the five health regions developed formal national guidelines ${ }^{2}$, which were subsequently revised in $2007^{3}$. After consultation with Vestfold Hospital Trust, the Southern Norway RHA agreed to establish a project offering morbidly obese patients a complete diagnostic assessment and treatment. The Morbid Obesity Center (MOC) (Overvektsprosjektet i Helse Sør) at Vestfold Hospital Trust was thereafter established on the $1^{\text {st }}$ September 2004. In June 2005 the former Hospital for Rehabilitation, now entitled Clinic for Physical Medicine and Rehabilitation (CFMR) at Vestfold Hospital Trust, initiated in cooperation with MOC the development of the specific outpatient intensive lifestyle intervention (ILI) program discussed in this thesis. When initiated, this was one of the first outpatient treatment programs for morbidly obese patients within the Norwegian tertiary health care sector. The choice of a group-based ILI-program was partly based on existing evidence indicating that group-based treatment was a more effective treatment of morbid obesity than individual treatments ${ }^{4}$. In part, the choice was also a result of practical considerations. During and after the completion of the studies presented and discussed in this thesis, the ILI treatment program at CFMR has become an established part of the non-surgical treatment at the MOC at Vestfold Hospital Trust. 
In order to manage the increasing prevalence of morbid obesity ${ }^{5}$ and the accompanying increase in obesity related comorbidities ${ }^{6}$ there must be both effective preventive measures and effective treatments available for patients. The number of bariatric operations performed worldwide has increased dramatically during the last decade ${ }^{7}$. Norway is no exception, with around 1500 bariatric operations performed in 2008 and around 3000 operations performed annually to date ${ }^{8}$. In addition, according to recent findings, more than one fifth of the population is obese $\left(\mathrm{BMI} \geq 30 \mathrm{~kg} / \mathrm{m}^{2}\right)$ and approximately $4-6 \%$ has a $\mathrm{BMI} \geq 35 \mathrm{~kg} / \mathrm{m}^{2}$. Assuming that approximately $50 \%$ of individuals with $\mathrm{BMI} \geq 35 \mathrm{~kg} / \mathrm{m}^{2}$ have one or more obesity related comorbidities, about $2-3 \%$ of the Norwegian population may be morbidly obese $^{9}$. Over the last ten years more than 4000 morbidly obese patients have sought treatment at the MOC, representing approximately $20 \%$ of the individuals with morbid obesity in our region (Former South Norway Regional Health Authority; Vestfold, Telemark, Buskerud, Aust-Agder, Vest-Agder) of approximately one million inhabitants. Of these treatment seeking patients less than half were offered bariatric surgery. The others were offered nonsurgical treatment alternatives including various individual and group-based ILI programs (figure 1).

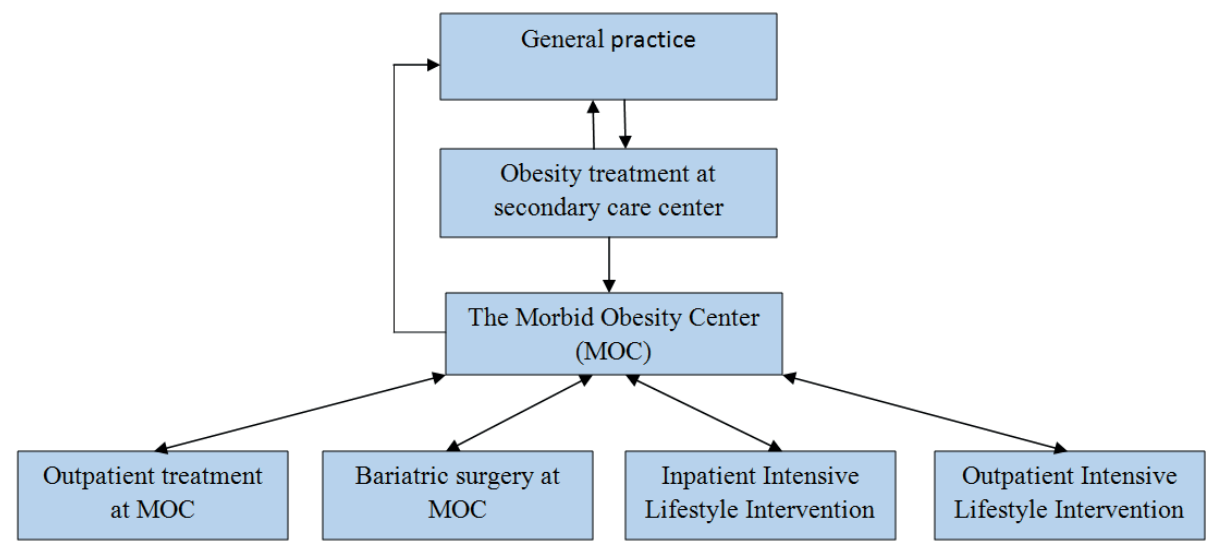

Figure 1. Flow chart of treatments at the Morbid Obesity Center. No permission required. 
While the effect of bariatric surgery on various health measures was established before the start of this PhD-project, less was known about the effects of ILI on weight loss, cardiovascular risk factors and health related quality of life (HRQL). Accordingly, we decided to study and compare the effectiveness of newly developed intensive lifestyle intervention programs, on morbidly obese individuals or similar patient groups (e.g. patients with type 2diabetes), assuming that the results might be generalized to future treatment seeking morbidly obese individuals.

The key issues in this thesis are threefold; in the first paper we compare the effectiveness of two public (secondary vs. tertiary care) outpatient lifestyle intervention programs in terms of weight loss, HRQL and physical activity. In order to compare the effectiveness of these two lifestyle interventions we used a non-randomized controlled pragmatic clinical design. In the second paper we compare the short-term effect of ILI and a low-calorie diet (LCD) in terms of aortic arterial stiffness (a valid surrogate marker of cardiovascular disease). Finally, in the third paper we compare the long-term effect of ILI on aortic arterial stiffness and compare this with the effect of a lifestyle intervention consisting of LCD and Roux-en-Y gastric bypass surgery (GBS).

All the studies in this thesis were conducted with treatment seeking morbidly obese patients. The results of the first paper can be seen to provide evidence which may help to inform decisions regarding the design and content of lifestyle interventions in public specialist health care systems. The results from the second and third paper, in addition to adding to our understanding of the short- and long term effects of various weight loss strategies on a surrogate marker of cardiovascular disease, also provide evidence which can be used to inform decisions regarding the design and content of lifestyle interventions with or without bariatric surgery in public specialist health care systems. 


\subsection{Obesity}

\subsubsection{Definition of obesity}

Obesity is a medical condition in which excess body fat increases to the extent that it may have negative consequences for individual health ${ }^{10}$. The most common classification of the degree of obesity is the body mass index (BMI), defined as body weight divided by height squared, expressed as $\mathrm{kg} / \mathrm{m}^{211}$. A high BMI brings with it an increased risk of comorbidities (Table 1), while the risk of several health problems also increases compared with a normal weight person (Table 2).

Table 1. The World Health Organization's (WHO) classification of obesity ${ }^{10}$.

\section{BMI $\left(\mathrm{kg} / \mathrm{m}^{2}\right) \quad$ Risk of comorbidities}

Underweight

$$
<18.5
$$

Low

Normal

$18.5-24.9$

Average

Overweight

$$
\geq 25.0
$$

Preobese

$25.0-29.9$

Increased

Obese class I

$30.0-34.9$

Moderate

Obese class II

$35.0-39.9$

Severe

Obese class III

$\geq 40.0$

Very severe

A definition of the term "morbid obesity" appeared for the first time in the American Journal of Surgery in 1969 when Scott and Law stated: “We identify morbid obesity as existing in any person whose weight has reached a level two or three times his ideal weight and who has maintained this level of obesity for five years or more despite efforts by himself, family, friends and physicians to bring about effective and sustained reduction of weight to medically acceptable standards"12. However, the term "ideal weight" was not specified, but assuming a 
BMI of $20 \mathrm{~kg} / \mathrm{m}^{2}$ as normal or ideal, a BMI of $40-60 \mathrm{~kg} / \mathrm{m}^{2}$ might be considered to be an indicator of morbid obesity. Currently we use the National Institute of Health Consensus Development Conference Panel's definition of morbid obesity, also referred to as clinically severe obesity, as either BMI $\geq 35 \mathrm{~kg} / \mathrm{m}^{2}$ with at least one obesity related comorbidity or BMI $\geq 40 \mathrm{~kg} / \mathrm{m}^{2} 13,14$.

Table 2. Relative risk of health problems associated with obesity ${ }^{10}$.

\begin{tabular}{lll}
\hline Greatly increased (relative & Moderately increased & Slightly increased (relative \\
risk greater than 3) & (relative risk 2-3) & risk 1-2) \\
\hline Type 2 diabetes & Coronary heart disease & Cancer (breast cancer in \\
& postmenopausal women, \\
Gallbladder disease & endometrial cancer, colon \\
Hypertension & cancer) \\
Dyslipidaemia & Reproductive hormone \\
Insulin resistance & abnormalities \\
Breathlessness & Pyperuricaemia and gout & Impaired fertility \\
Sleep apnoea & Low-back pain due to obesity \\
& & Increased risk of anaesthesia \\
& complications \\
& & Fetal defects associated with \\
& & maternal obesity \\
& &
\end{tabular}




\subsubsection{Obesity trends/prevalence}

Obesity is caused by either a higher energy intake or a lower energy expenditure (or a combination of both) than is needed for a stable weight, and is often driven by genetic susceptibility. Changes in the global food system, especially increasing food energy supply, combined with increased mechanization and motorization are major drivers of the rise in global obesity seen over the last 3 to 4 decades ${ }^{15}$. Parallel with increased standards of living, obesity as result of over-nutrition and physical inactivity has become a major threat to the public health worldwide ${ }^{16}$. In fact, overweight has become more of a problem than underweight and infectious diseases in many developed countries ${ }^{10}$. While some reports indicate that the prevalence of obesity has stabilized during the last decade, a recent study showed the prevalence of overweight and obesity to be increasing worldwide. Between 1980 and 2013 the prevalence of overweight and obesity rose by $27.5 \%$ for adults, and $47.1 \%$ for children. Approximately one third of the world population, (2.1 billion people) can be defined as either overweight or obese, with 671 million defined as obese ${ }^{17}$. There is also evidence that the prevalence of morbid obesity is increasing at a high rate within the obese population $^{5,18,19}$. During the last decade the prevalence of a BMI $>40 \mathrm{~kg} / \mathrm{m}^{2}$ has grown at twice the rate of moderate obesity, while the prevalence of a BMI $>50 \mathrm{~kg} / \mathrm{m}^{2}$ has a grown threefolds compared to the increase in moderate obesity ${ }^{5}$. The prevalence of obesity among men and women aged 40-44 years has increased in Norway over the last three to four decades (Figure 2). Data from Norway demonstrate an increase in the prevalence of obesity at all ages and within both genders, especially among young adults ${ }^{9}$. Recent research on obesity in Norway demonstrates that approximately $58 \%$ of men are overweight or obese, while $19 \%$ are obese. In women the prevalence of overweight or obese is approximately $47 \%$, with $18 \%$ obese $^{17}$. 


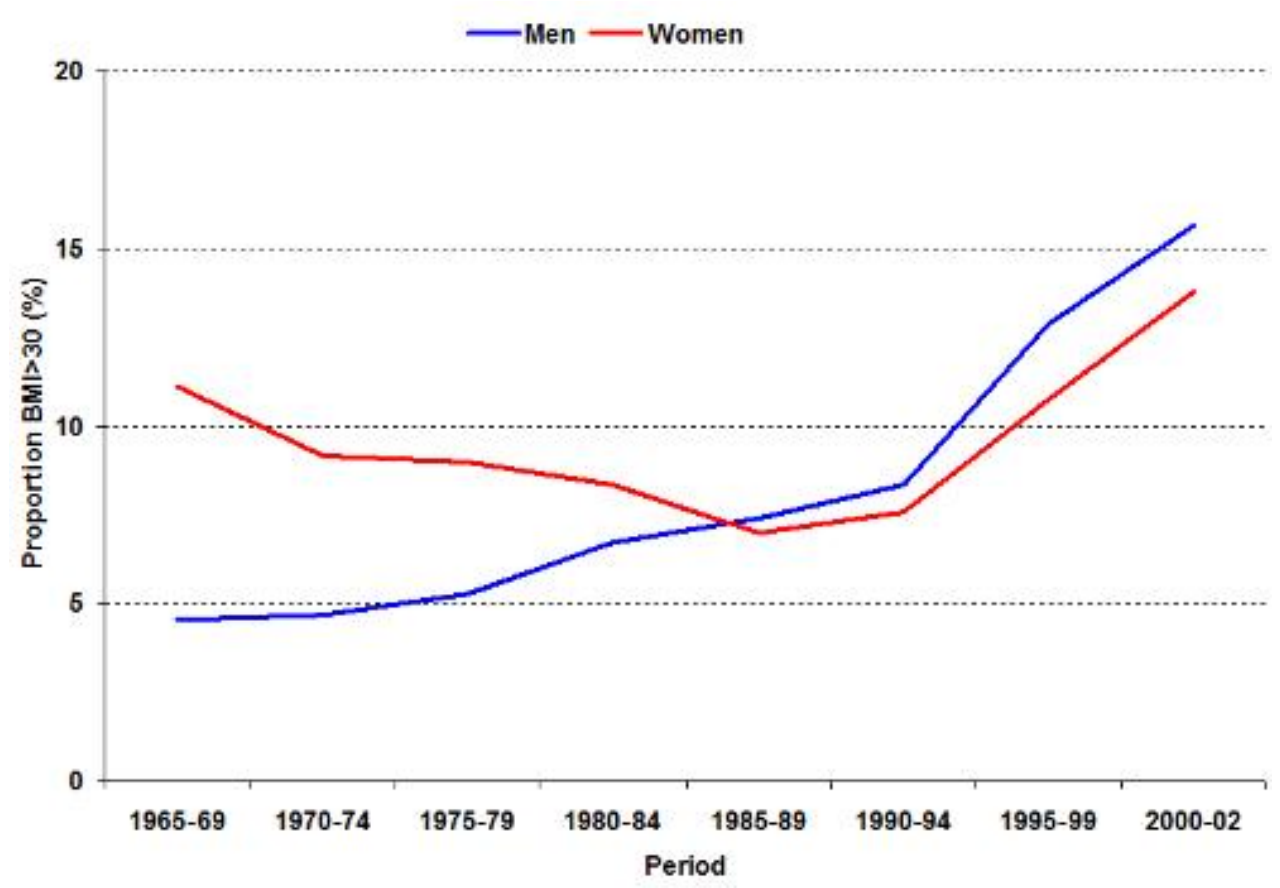

Figure 2. Proportion of Norwegian women and men aged 40-44 years with a BMI $\geq 30 \mathrm{~kg} / \mathrm{m}^{2}$ in the period 1965 to $2000^{20}$.

\subsection{The burden of obesity}

The burden of disease refers to the impact of a health problem measured in terms of financial cost, mortality or morbidity. According to the $\mathrm{WHO}^{21}$, obesity, hyperglycemia, high blood pressure, tobacco usage and physical inactivity represent the five leading global risks to mortality. As a country develops there is a shift from a risk of primarily infectious diseases to a risk of primarily non-communicable diseases (Figure 3). Further, an increase in obesity levels is also associated with substantial increases in treatment costs related to the many obesity-related diseases ${ }^{6}$. 


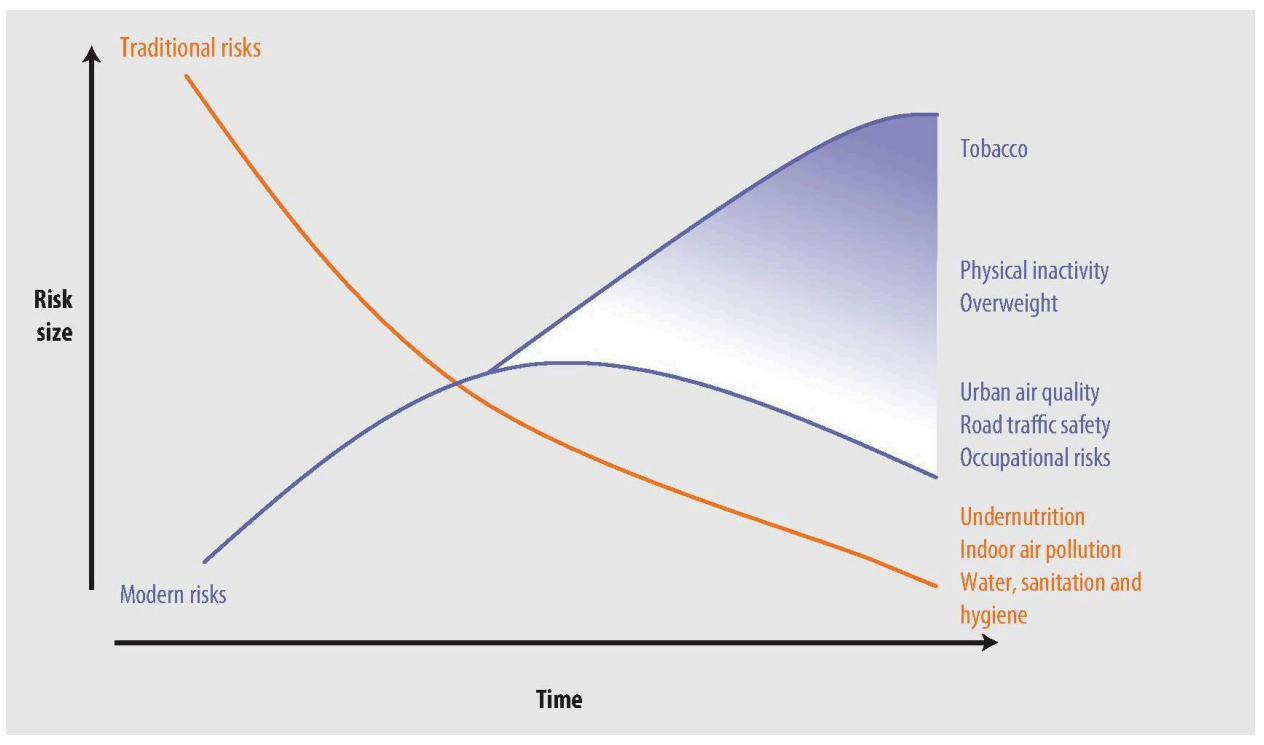

Figure 3. The risk transition. Over time, major risks to health shift from traditional risks (e.g. inadequate nutrition or unsafe water and sanitation) to modern risks (e.g. overweight and obesity). Modern risks may take different trajectories in different countries, depending on the risk and the context ${ }^{21}$.

The positive association between BMI and all-cause and cardiovascular mortality is documented in numerous studies ${ }^{22-28}$. Further, there are also associations between obesity and increased risk of coronary heart disease ${ }^{29,30}$, metabolic syndrome ${ }^{31}$, physical inactivity ${ }^{32}$, cancer $^{33}$, urinary incontinence ${ }^{34}$, gastro-esophageal reflux ${ }^{35}$, obstructive sleep apnea ${ }^{36}$, osteoarthritis $^{37}$, depression ${ }^{38}$ and HRQL ${ }^{39}$. Both dietary and surgically induced weight loss improve the risk of cardiovascular disease in morbidly obese individuals in terms of reduced blood pressure, blood glucose and lipid levels ${ }^{40}$. Further, increased aerobic fitness and physical activity improve the risk of cardiovascular disease independent of weight loss, especially in unfit overweight and obese individuals ${ }^{41}$. The number of excess deaths caused 
by obesity has been estimated at approximately 30000 in the UK, 300000 in the USA ${ }^{42,43}$ and 3.4 million worldwide ${ }^{44}$. Higher mortality rates among obese subjects seem to mainly be explained by obesity related comorbidities like type 2 diabetes and hypertension rather than by obesity alone ${ }^{45}$. Because of the increased health risk associated with obesity and the large increase in its prevalence, obesity has now become a major global health challenge ${ }^{17}$.

\subsubsection{Aortic arterial stiffness}

Aortic stiffness provides information on risk of cardiovascular disease beyond that provided by traditional risk factors ${ }^{46}$, and represents the accumulated damaging effects of these factors over time $\mathrm{e}^{47,48}$

In the early 1920's Frank, Bramwell and Hill derived the Moens-Korteweg equation, illustrating that the propagation of the pulse wave along the arterial three is inversely related to the distensibility of the arterial tube ${ }^{49}$. When the heart contracts it generates a pulse/wave which moves around the circulatory system. The aortic vessels cause a cushioning-effect on the pulse wave and transform the periodical left ventricular ejection of blood into a continuous flow. This is made by the release through elastic recoil of temporary stored energy with every heartbeat during the systole ${ }^{50}$. The speed of this pulse wave (pulse wave velocity $(\mathrm{PWV}))$ is related to the stiffness of the arteries ${ }^{49}$. An increase in PWV causes a premature return of reflected waves in late systole with increasing central pulse pressure and thus systolic blood pressure. An increase in systolic blood pressure causes an increased load on the left ventricle with increasing myocardial oxygen demand, and is associated with left ventricular hypertrophy. Toto-Moukouo et al. were the first to evaluate the mechanical properties of large arteries in obese individuals, reporting that arterial stiffness increased independent of age, gender and blood pressure level ${ }^{51}$. 
When matched for age, aortic PWV is approximately $0.5 \mathrm{~m} / \mathrm{s}$ higher in obese persons than in non-obese persons. This difference in aortic PWV is equivalent to 5 to 10 years of aging ${ }^{52}$. Arterial stiffness and hypertension are closely related, with aortic stiffness and arterial pulse wave reflections key determinants of elevated central systolic pressure ${ }^{49}$.

\subsubsection{Physical inactivity}

Obesity is associated with reduced physical activity levels and increased sitting time. The term physical activity refers to any body movement that burns calories and includes work, play, daily chores and the daily commute ${ }^{53}$. Some studies find that levels of exercise, leisure time and physical activity have remained stable/ increased slightly over the last few decades $^{54-56}$, albeit that these activities only represent a small part of daily physical activity. The main contributors to daily physical activity: activity at work, home and during transportation have declined due to economic growth, technological advancements and social changes $^{54,55,57}$. While high levels of physical activity reduce the health risk of obesity ${ }^{58}$,

physical inactivity is associated with increased obesity and all-cause mortality ${ }^{59}$. However, it is unclear if physical inactivity is a cause or a consequence of obesity. One can argue that obesity most likely makes it harder to engage in physical activity, while reduced physical activity levels can contribute to a further weight gain and thus produce a vicious circle. Further, there is some degree of uncertainty regarding exactly how much physical activity is needed to avoid weight gain ${ }^{60}$.

\subsubsection{Health related quality of life}

While quality of life refers to how well an individual functions in daily life and his/hers perceived well-being ${ }^{61}$, the most common understanding of HRQL is that it is a multidimensional measurement of the impact an illness and/or its treatment has on individuals ${ }^{62}$. 
HRQL is usually measured with either generic or disease-specific instruments. While the generic instruments are designed to measure HRQL across divergent populations, the diseasespecific instruments are designed to measure HRQL in a defined population. In clinical studies it is recommended that both types of instruments are applied ${ }^{63}$. Obesity is associated with impaired HRQL ${ }^{63-66}$, with improving HRQL considered to be an important treatment goal. While some studies have found that weight reduction is associated with improved $\mathrm{HRQL}^{67-69}$, a recent meta-analysis demonstrated only modest improvements in the physical component of HRQL, with no improvement in the mental component ${ }^{70}$.

A recent study has demonstrated a relationship between physical activity levels and parts of HRQL (life satisfaction and physical function, but not mental function) in a group of severely obese persons prior to their undertaking a lifestyle intervention program ${ }^{71}$. Further, another study has demonstrated a dose dependent relationship between exercise dose and improvements of HRQL in obese sedentary post-menopausal women ${ }^{72}$. However, other studies have found that physical exercise alone does not improve HRQL, with improvement in HRQL occurring only when combined with a dietary intervention ${ }^{73}$.

\subsection{Obesity treatment strategies}

The cornerstone of obesity management is weight loss, with two concomitant principles being that the treatment of obesity should reduce energy intake and increase energy expenditure through lifelong behavioral changes. In order to achieve benefit with respect to cardiovascular disease (CVD) risk factors, morbidity and mortality guidelines from US and UK recommend a 5-10\% weight reduction ${ }^{74-76}$, while others, including the Norwegian guidelines, suggest that patients with a BMI $\geq 35 \mathrm{~kg} / \mathrm{m}^{2}$ with comorbidities should achieve a greater weight loss in order to obtain a consistent improvement in comorbidities ${ }^{75,77,78}$. 


\subsubsection{Guidelines for the treatment of obesity in Norway}

According to Norwegian guidelines, obesity treatment should start at the primary care level. The general practitioner (GP) should, together with the patient, set realistic and relevant treatment objectives. Further, the GP, in coordination with dieticians and physiotherapists/physical educators, should offer an individualized weight loss program based on a healthy diet with energy restriction and an increased level of physical activity. The GP coordinates the treatment and decides who should be referred to an outpatient center in a secondary care clinic for more specialized treatment. If needed, morbidly obese patients can be referred from the secondary care clinic to a tertiary care center with multidisciplinary teams specializing in treatment of morbidly obese patients ${ }^{77}$. These tertiary care centers are obliged to offer either bariatric surgery or a lifestyle intervention program, both of which are part of, and funded by, the Norwegian public health service. The patient and the physician together agree upon the most appropriate choice of therapy; either surgical or conservative ${ }^{79}$.

\subsubsection{Physical activity}

The terms "Physical activity" and "exercise" are often used interchangeably, though clinical practice operates with differing definitions of the two. While the term physical activity refers to any body movement that burns calories, exercise is usually defined as a subcategory of physical activity, and refers to planned, structural and repetitive activities aimed at improving physical fitness and/or health ${ }^{53}$.

The intensity of a specific physical activity or exercise can be defined as the ratio of work metabolic rate to resting metabolic rate (Metabolic Equivalent of Task [MET]. One MET is considered the energy cost for sitting quietly, approximated for an average adult to $3.5 \mathrm{ml}$ oxygen per kg body weight per minute or $1 \mathrm{kcal}$ per $\mathrm{kg}$ body weight per hour ${ }^{80,81}$ 
There is a large body of evidence suggesting that high levels of physical activity or a high cardio respiratory fitness reduce the health risk associated with obesity ${ }^{41,58,82-84}$. Physical activity and exercise are associated with reduction ${ }^{85}$ and maintenance ${ }^{86}$ of body weight, but cause only a modest weight loss without energy restriction ${ }^{87,88}$. Interventions combining energy restriction and physical activity result in a larger weight loss than energy restriction alone, but this weight loss is in many cases only partly sustained after 1 year $^{89}$. There is a dose-response relationship between physical activity level and weight $\operatorname{loss}^{90}$, and vigorous physical activity seems to be more effective for weight control than slow walking $^{91,92}$. The effects of physical activity in preventing weight gain are modest, especially

since adherence to a prescribed exercise program is a big challenge for obese persons ${ }^{84}$. There is evidence that 30 minutes moderate intensive activity daily limits the health risk of a number of chronic diseases, including coronary heart disease and diabetes ${ }^{89}$. The recently published Norwegian guidelines for physical activity suggest that adults and the elderly should perform physical activity with moderate intensity for at least $150 \mathrm{~min} /$ week, or at least $75 \mathrm{~min} /$ week with intensive physical activity. Further, it is pointed out that $300 \mathrm{~min} /$ week with moderate physical activity or $150 \mathrm{~min} /$ week with intensive physical activity will lead to additional health benefits. In addition, the general population should reduce their physical inactivity or sitting time to a minimum ${ }^{93}$. However, there is compelling evidence that $60-90$ minutes of moderate or lesser amounts of vigorous activity daily is required in order to prevent weight regain in formerly obese individuals ${ }^{94}$.

\subsubsection{Behavioral change}

Traditionally, behavior intervention in obesity management has been based on the assumption that behaviors regulating body weight are learned, and thus can be relearned or modified ${ }^{95}$. Currently, researchers operate on the assumed basis that body weight is also affected by 
factors other than behavior. These include genetic, metabolic and endocrine influences ${ }^{96}$. In order to promote behavioral changes a variety of techniques can be used. Behavioral intervention is a generic term for those approaches used to help individuals develop skills through which they can achieve behavioral changes ${ }^{96}$. Behavioral intervention is goal directed, process oriented and advocates small rather than large changes ${ }^{96,97}$. It usually consists of some common components; self-monitoring, eating stimulus control, nutrition education, slower eating, physical activity, problem solving and cognitive restructuring ${ }^{95,96}$. One often used technique to change behavior is Motivational Interviewing, which focuses on facilitating and engaging the latent intrinsic motivation within the client in order to change behavior. Further, Motivational Interviewing is a goal-oriented and client-centered counseling style which promotes behavior change by exploring and resolving client ambivalence ${ }^{98}$.

\subsubsection{Lifestyle intervention without bariatric surgery}

There are a great variety of lifestyle interventions used in the treatment of overweight and obesity. The cornerstone of lifestyle intervention is to increase physical activity and normalize eating patterns. The interventions include a variety of individual and group based treatment options offered by general practitioners, private institutions, local hospitals, tertiary care centers and various weight loss camps. The interventions are organized as out- or inpatient treatment with varying intensity of treatment. Key features for good long-term results include compliance with physical activity regimes, caloric restriction, self monitoring of weight and regular personal contact during the weight maintenance period ${ }^{99,100}$. All of the above aim to induce a long-term weight loss of at least 5-10\% ${ }^{77}$. Lifestyle interventions combining diet and exercise are associated with a larger weight loss compared to diet alone ${ }^{87}$. Studies of overweight and obese individuals using a combination of physical activity and dietary interventions led to a 4-6 \% weight loss after three to four years ${ }^{101-103}$, while one study reports 
a weight loss of $6 \%$ after approximately 10 years ${ }^{104}$. Results from studies of morbidly obese individuals have shown 5-20\% weight reduction after 5-24 months ${ }^{105-112}$. However, other long-term studies have demonstrated that two thirds of the recorded weight losses were regained in the follow-up period ${ }^{105}$.

\subsubsection{Lifestyle intervention with bariatric surgery}

Bariatric surgery has continued to evolve and develop since the 1950's, and includes a variety of procedures ${ }^{113-115}$. Bariatric surgery is generally categorized as restrictive, malabsorptive or a combination of restrictive and malabsorptive ${ }^{116}$. Adjustable gastric banding, sleeve gastrectomy, and vertical banded gastroplasty are all categorized as restrictive operations ${ }^{116}$. Biliopancreatic diversion, jejunoileal bypass and jejunocolic bypass are categorized as malabsorptive procedures. Biliopancreatic diversion/duodenal switch and gastric bypass surgery are categorized as combinations of restriction and malabsorption ${ }^{116}$. However, there is also considerable evidence demonstrating that these procedures induce satiety and less hunger through their effect on the gut hormones ${ }^{116}$. The restrictive effect of bariatric surgery causes patients to feel full at an earlier period during the eating of meals. Accordingly, bariatric surgery may help the patients to change their eating habits, e.g. by eating smaller portions. Gastric bypass surgery (GBS) was developed by Mason and Ito in the 1960's ${ }^{117}$ (Figure 4) and is associated with a $25 \%$ mean long-term weight loss ${ }^{118}$. GBS accounts for almost $50 \%$ of all bariatric surgery performed worldwide ${ }^{7}$, and for approximately $90 \%$ of all bariatric surgery performed in Norway ${ }^{119}$. 


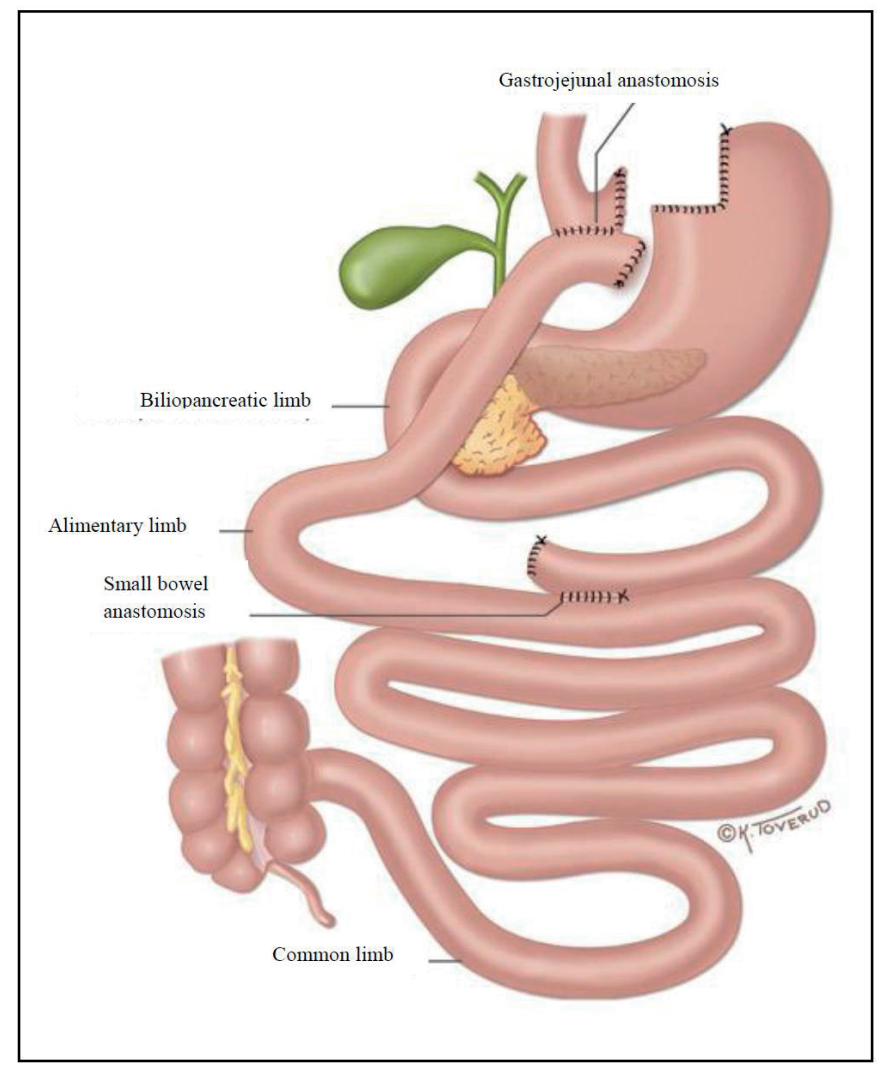

Figure 4. Illustration of a Roux-en-Y gastric bypass. Illustration reproduced with permission from (C) Kari C. Toverud CMI (certified medical illustrator) from Hofsø et al. ${ }^{109}$

\subsection{Summary}

Obesity is one of the main medical challenges of our time. Although the cornerstone of treatment is reduced energy intake and increased energy expenditure, greater understanding of safe and effective morbid obesity treatments is needed. The number of patients undergoing bariatric surgery is relatively high, yet it remains unclear what long-term adverse effects there $\operatorname{are}^{120}$. Further, the National Council for Priority Setting in Health Care in Norway has recommended that the development of high quality safe and effective conservative treatment programs should be prioritized ${ }^{121}$. 
The main aims of this thesis are firstly to compare the effectiveness of two existing morbid obesity lifestyle interventions in terms of weight loss, health related quality of life and physical activity level. Second, we compare the short term (7-weeks) effect of a low calorie diet and intensive lifestyle intervention on aortic arterial stiffness. Finally, we compare the long-term (1-year) effects of gastric bypass surgery and intensive lifestyle intervention on aortic arterial stiffness. 


\section{Chapter 2. Aims of thesis}

Paper I:

In treatment seeking morbidly obese individuals we aimed to compare the effectiveness of an intensive lifestyle intervention program (tertiary care level) and a moderate lifestyle intervention program (secondary care level) in terms of body weight, physical activity level and health related quality of life.

\section{Paper II:}

We aimed to investigate and compare the 7-week effectiveness of a low calorie diet and an intensive lifestyle intervention program on arterial stiffness in treatment seeking morbidly obese individuals.

\section{Paper III:}

We aimed to investigate and compare the 59-week effectiveness of an intensive lifestyle intervention program and a low calorie diet followed by a Roux-en-Y gastric bypass surgery intervention on arterial stiffness in treatment seeking morbidly obese individuals. 


\section{Chapter 3. Research design and methods}

\subsection{Trial designs}

Paper I is based on the results from a non-randomized controlled pragmatic clinical trial. In the original protocol (ClinicalTrials.gov identifier NCT00464984), GP's in the county of Vestfold were to recruit and treat patients within the moderate lifestyle intervention (MLI) program. Despite several efforts, the GP who initially accepted our invitation to participate in the study did not recruit a sufficient number of patients. During the period from August 2006 to November 2009 a total of 13 patients were referred to the MLI-group. Since GP's preferred to refer the patients to our secondary care outpatient clinic, we found it appropriate to change the protocol and recruit consecutive patients from our secondary outpatient clinic to the MLIgroup. The recruitment to the MLI-group from our secondary outpatient clinic started in January 2010. The original study and revised protocol were approved by the regional ethics committee of the Southern Norway Regional Health Authority (identifier S-05175).

Paper II and III are based on short- and long-term results from a non-randomized clinical trial, but we would argue that this study also had a pragmatic design according to the CONSORT statement $^{122,123}$. All of the interventions in this study were performed in a normal treatment setting at Vestfold Hospital Trust, with all patients recruited from MOC and a relatively low number of excluded patients.

\subsection{Settings, intervention overview and participants}

Settings and principles of interventions. In the study presented in paper I, patients were either assigned to an ILI program (at CPMR, Vestfold Hospital Trust, Stavern, Norway) or to an MLI program (Vestfold Hospital Trust, Larvik, Norway) (Figure 5). Patients were assigned to 
the ILI-group between August 2006 and November 2010, while patients were assigned to the MLI-group between January 2010 and November 2010.

The studies presented in paper II and III were performed at a tertiary care center (The MOC, Vestfold Hospital Trust, Tønsberg, Norway) between February 2008 and April 2012. In this non-randomised trial, patients were either assigned to ILI (at the CPMR, Vestfold Hospital Trust) or to a LCD followed by GBS at the MOC (Figure 6).

Participants. In paper I, all participants in the ILI-group were recruited from the MOC while all the patients in the MLI-group were referred to our secondary care treatment center by GPs in the county of Vestfold. In order to be included in the study, a patient had to be considered morbidly obese $\mathrm{e}^{13,14}$.

Subjects were excluded from the study if they had participated in a weight loss program during the previous 12 months, if they had a severe psychiatric disorder preventing participation in a group-based intervention, or if they were not able to walk more than 100 meters without the use of a walking aid.

In paper II and III, all participants were recruited from the MOC and had to reside within 100 $\mathrm{km}$ from either CPMR or MOC. Patients in the ILI-group were selected from patients that had registered for participation in a standardized lifestyle intervention at the CPMR. Participants in the LCD-group, and later GBS-group, were selected from patients due to undergo bariatric surgery at Vestfold Hospital Trust.

In order to be included in the study patients had to be considered morbidly obese. The exclusion criteria included uncompensated heart failure, cardiac arrhythmias, unstable angina, end-stage renal disease, known bleeding disturbances, serious psychiatric disorders, serious eating disorders, cardiac pacemakers, intra-cardiac devices, a cerebrovascular event or a myocardial infarction within the last six months. 


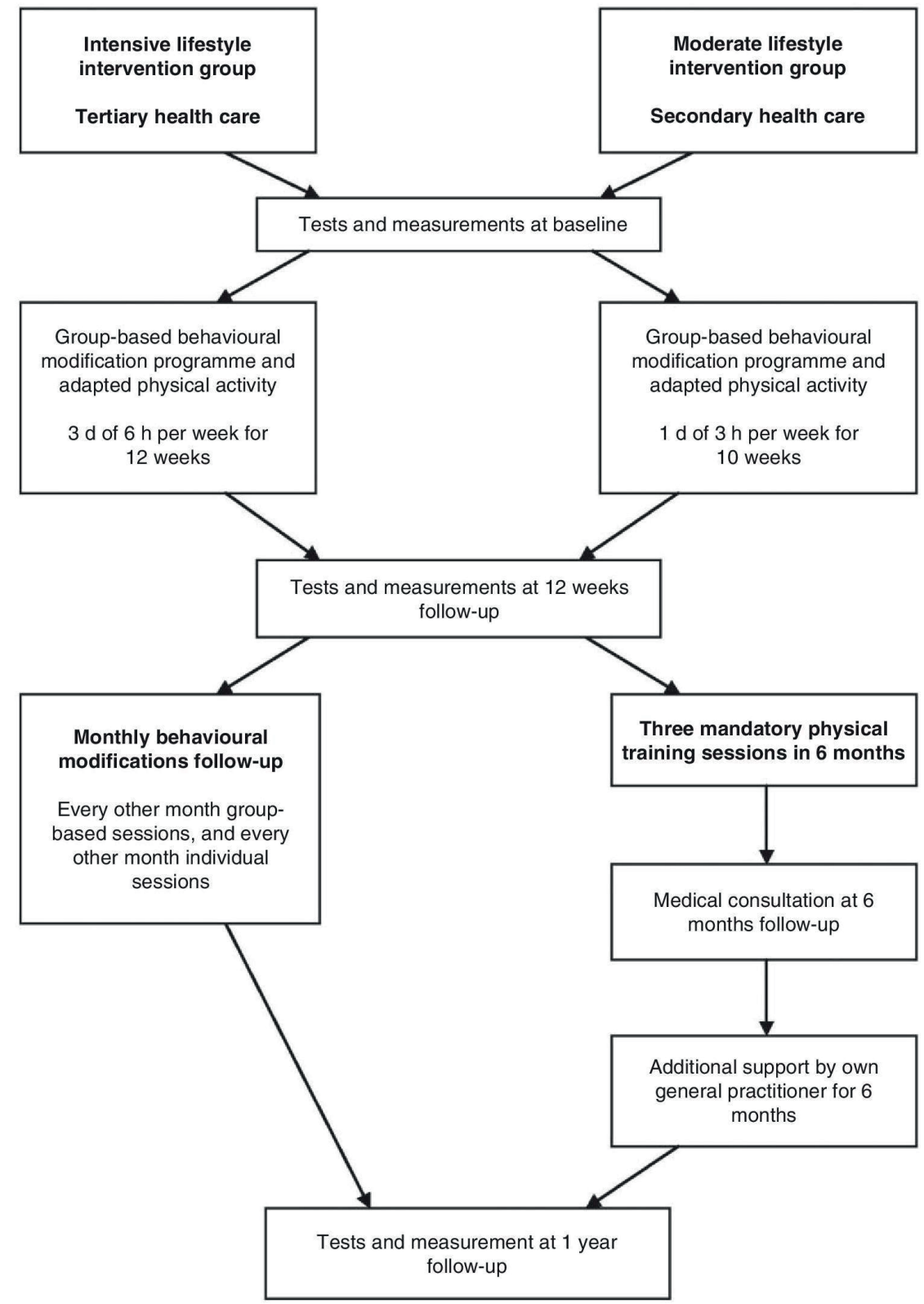

Figure 5. Chart of interventions in paper I. No permission required ${ }^{124}$. 


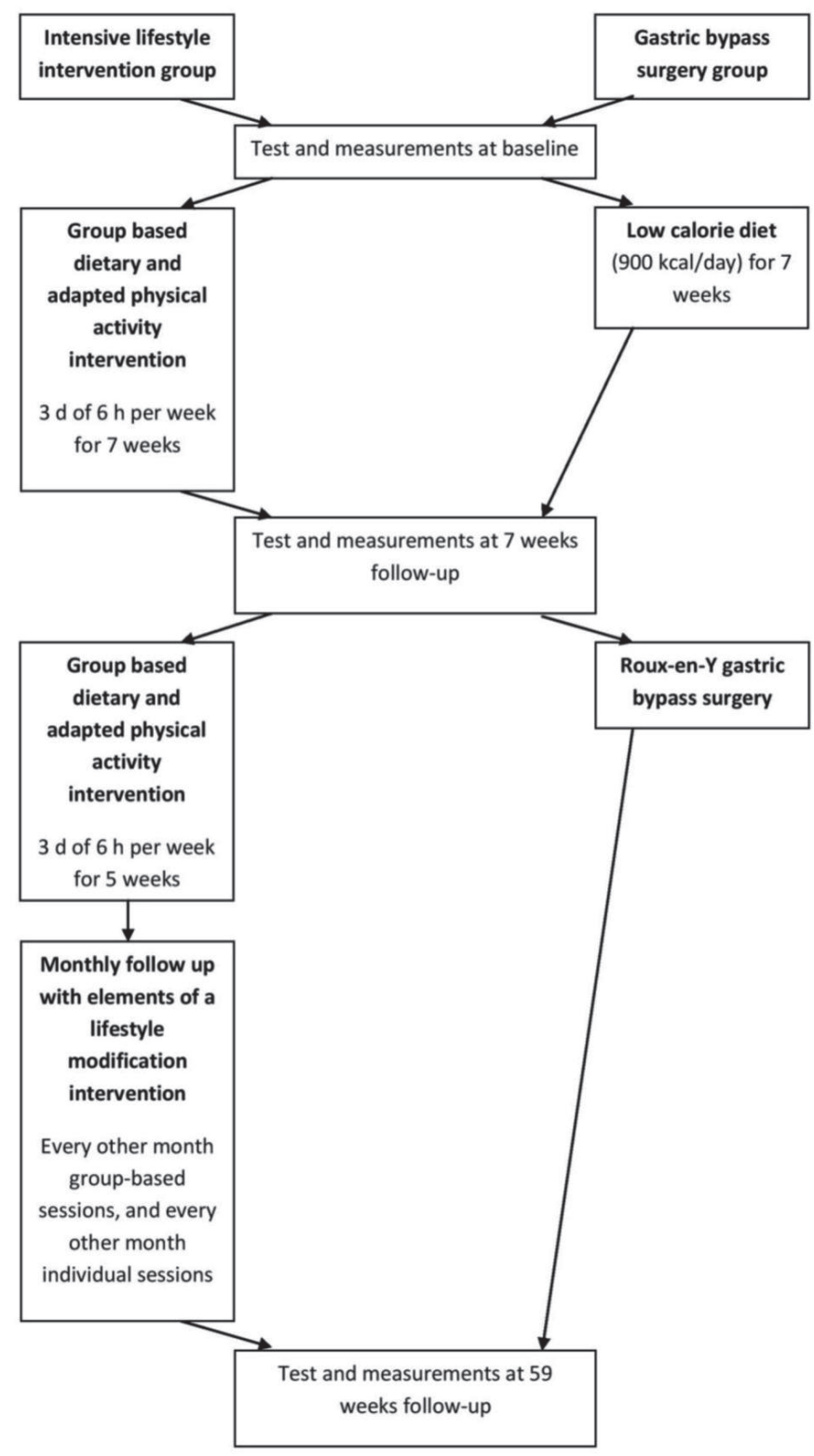

Figure 6. Chart of interventions in paper II \& III. No permission required ${ }^{125}$. 


\subsection{Interventions (details)}

\subsubsection{Intensive lifestyle intervention program}

The ILI program was conducted at "Kysthospitalet i Stavern”, which provided participants for all the papers discussed in this thesis. The program included both dietary energy restrictions and physical activities. The participants were divided into groups comprising of 12 to 14 patients. The first year of the intervention was divided into two stages: the first 12 weeks included the most intensive treatment period, with treatment sessions 3 days per week for 12 weeks. In the second part of ILI, during weeks 13-52, patients received monthly follow up, alternating between group based and individual sessions every other month (figure 5). During these group-based sessions, which lasted for 3 hours, patients performed various supervised water-based endurance exercises for the first hour, followed for the next 2 hours by a lifestyle modification intervention including nutritional and physical activity lectures (Figure 7).

\section{Dietary energy restriction}

The baseline individual energy expenditure was calculated according to Schofield's equation $^{126}$. Schofield's equation uses a patient's age, gender and body weight in order to calculate the basal metabolic rate (BMR). Assuming that most of the severely obese participants had a sedentary lifestyle ${ }^{127}$, the individual baseline energy expenditure was multiplied with a physical activity factor of $1.4^{80,81}$. Patients received a dietary plan with a reduced energy intake of $1000 \mathrm{kcal} /$ day of the calculated total energy expenditure, with a specific aim of at least $5-10 \%$ weight $\operatorname{loss}^{77}$. Otherwise, the diet compiled with Norwegian nutritional guidelines ${ }^{128}$. 


\begin{tabular}{|c|c|c|c|}
\hline \multicolumn{4}{|c|}{ Time Schedule - Intensive Lifestyle Intervention - stage 1. 1-3 monthts } \\
\hline & Monday & Tuesday & Thursday \\
\hline 09:00 & \multirow{2}{*}{ Physical activity } & \multirow[t]{3}{*}{ Walking, Nordic walking } & \multirow[t]{3}{*}{ Physical activity } \\
\hline 09:30 & & & \\
\hline 10:00 & \multirow{2}{*}{$\begin{array}{c}\text { Body weight and } \\
\text { anthropometric measures }\end{array}$} & & \\
\hline $10: 30$ & & Education* $^{*}$ & \multirow[t]{2}{*}{ Education* $^{*}$} \\
\hline 11:00 & Education* & \multirow[t]{2}{*}{ Lunch } & \\
\hline 11:30 & \multirow[t]{2}{*}{ Lunch } & & \multirow[t]{2}{*}{ Lunch } \\
\hline $12: 00$ & & \multirow[t]{4}{*}{ Water activities } & \\
\hline $12: 30$ & \multirow[t]{3}{*}{ Education* $^{*}$} & & \multirow[t]{3}{*}{ Education* $^{*}$} \\
\hline 13:00 & & & \\
\hline 13:30 & & & \\
\hline 14:00 & \multirow[t]{2}{*}{ Water activities } & \multirow[t]{2}{*}{ Education* } & \multirow[t]{2}{*}{ Water activities } \\
\hline $14: 30$ & & & \\
\hline 15:00 & End & End & End \\
\hline
\end{tabular}

Time Schedule - Intensive Lifestyle Intervention - group sessions - stage 2. 3-12 months

\begin{tabular}{|l|l|}
\hline $08: 45$ & Body weight and anthropometric measures \\
\hline $09: 30$ & Water activities \\
\hline 11:00 & Education \\
\hline 12:00 & End \\
\hline
\end{tabular}

* Education in physical activity, nutrition and motivation.

Figure 7. Time schedule for the first year of the intensive lifestyle intervention (No permission required) ${ }^{124}$.

\section{Physical activity}

During the first 12 weeks each treatment day lasted 6 hours, with patients participating in two supervised training sessions for 60-90 minutes (figure 7). This was followed by lectures on nutrition, physical activity and motivation. The first of the two supervised exercise sessions consisted of weight bearing activities such as walking, Nordic-walking, running, ballgames, resistance training and various other exercises. The second exercise session consisted of water-aerobics, swimming and other water-based physical activities. The main emphasis of 
the physical training was endurance training of moderate (4 METs) to high intensity (8 METs). The resistance training consisted of 10-15 repetitions and 2-3 sets per exercise.

\section{Behavioral change}

Group-based interventions seem to be more effective than solely individual treatments for

obesity ${ }^{4}$. In order to invoke behavioral changes, elements of cognitive behavioral therapy ${ }^{129}$ and motivational interviewing ${ }^{98}$ were used in both individual and group sessions. The program is mainly a group-based intervention, but with individual sessions in order to facilitate a more tailored treatment.

The treatment team included a registered nurse, a medical doctor and physical educators with backgrounds in nutrition, pedagogy and adapted physical activity, the latter of which focuses on the individual differences in physical activity that require special attention ${ }^{130}$.

\subsubsection{Moderate lifestyle intervention program}

The Moderate Lifestyle Intervention (MLI) program included outpatient treatment 1 day a week for 10 consecutive weeks, for 3 hours each day (figure 5). Through energy restriction and increased physical activity the specific treatment goal for each patient was a long-term weight loss of at least 5-10\% ${ }^{77}$. Each day of treatment included one session of supervised physical exercise such as aerobics, treadmill walking, step machines, indoor cycling and resistance training. The intensity of the physical endurance training was moderate (4 METs) to high (8 METs). Resistance training was performed with 10-15 repetitions and 2-3 sets per exercise. In addition, participants received lectures in nutrition, physical activity and motivation by the multidisciplinary team. During the first 10 weeks, patients participated in two water aerobic sessions. Participants were encouraged to increase their physical activity 
levels between sessions. Participants were encouraged to attend at least 8 of 10 treatment sessions.

The treatment staff in the MLI included a registered nurse, a physiotherapist, a registered dietician and a medical doctor.

\subsubsection{Low calorie diet}

The LCD consisted of a diet based on crisp bread combined with low fat products for breakfast, lunch and supper. Dinner consisted of fish, poultry of lean meat combined with potatoes, rice or pasta and vegetables. The diet provided less than $900 \mathrm{kcal}$ per day, of which roughly $40 \%$ of the energy came from protein, $40 \%$ from carbohydrates and $20 \%$ from fat (estimations based on calculations from a registered dietician, Line Kristin Johnson, $\mathrm{PhD}^{131}$ ). Patients were allowed to increase the size of their meal through vegetable intake, and were also encouraged to drink water when eating. Daily intake of a multivitamin/mineral supplement was recommended. Further, participants in the LCD-group were instructed to maintain their level of physical activity throughout the intervention. The study participants were not allowed to attend other dietary or physical interventions either prior to or during the 7 weeks of the LCD.

\subsubsection{Gastric bypass surgery}

The Roux-en-Y GBS was performed laparoscopically at Vestfold Hospital Trust, Tønsberg, Norway. The gastric pouch was about $25 \mathrm{ml}$, while the intestinal limb lengths were measured as follows: alimentary limb $120 \mathrm{~cm}$, biliopancreatic limb $60 \mathrm{~cm}$, and common limb, variable length. The following standardized regimen of dietary supplements was prescribed to all patients after surgery: 2 daily multivitamin/mineral pills, vitamin-D with calcium $\left(10 \mu \mathrm{g} \mathrm{D}_{3}\right.$ 
and $0.5 \mathrm{~g}$ Ca carbonate), ferrous sulphate (100 $\mathrm{mg}$ to women with menstruation). Additionally $\mathrm{B}_{12}(1 \mathrm{mg})$ was given intramuscularly 1 month after surgery, then every 3 months.

\subsection{Outcomes}

The outcomes of primary interest in paper I were 1-year changes in body weight, HRQL and physical activity level. The 1-year changes in waist circumference (WC) were also analyzed. In paper II and III the main outcome was change in arterial stiffness measured with ${ }_{\mathrm{cf}} \mathrm{PWV}$. Secondary outcome variables were changes in body weight, body composition, systolic and diastolic blood pressure and pulse pressure.

\subsubsection{Clinical characteristics and definitions}

In all papers discussed in this thesis the anthropometric measures were conducted with patients wearing light clothing and no shoes. Height was measured to the nearest $0.5 \mathrm{~cm}$, and body weight to the nearest $0.1 \mathrm{~kg}$. Body mass index (BMI) was calculated as weight in kilograms divided by the square of the height in meters. WC was measured midway between the $12^{\text {th }}$ rib and the iliac crest with the patient in an upright position.

The patients in paper II and III underwent a medical examination performed by a medical doctor and a trained nurse. All measurements were conducted after an overnight fast. Patients were instructed to withdraw prescribed medications and to refrain from smoking from 24:00 the evening prior to the examination. Blood was collected by venipuncture on the day of the medical examination. Resting blood pressure was measured using an electronic blood pressure recorder with an appropriately sized cuff (Dinamap ${ }^{\circledR}$, ProCare Series, GE. Medical Systems (Buckinghamshire, UK) with the patient sitting in an upright position. Three measurements were recorded, and the average of the second and third recording was used in the blood pressure analysis. Arterial hypertension was defined by either, a systolic blood 
pressure $\geq 140 \mathrm{mmHg}$, diastolic blood pressure $\geq 90 \mathrm{mmHg}$ or the use of antihypertensive medication. Mean arterial pressure (MAP) was calculated as ([diastolic blood pressure x 2] + systolic blood pressure) $/ 3^{52}$. Ischemic heart disease was defined as a history of stable coronary artery disease, percutaneous coronary intervention, coronary artery bypass graft surgery or myocardial infarction. Homeostasis Model Assessment Insulin Resistance (HOMA-IR) was calculated as ([fasting serum glucose $(\mathrm{mmol} / \mathrm{l}) \mathrm{x}$ fasting serum insulin (pmol/1)]/135 ${ }^{132}$. Low density lipoprotein cholesterol (LDL) concentrations were estimated by the Friedewald equation: LDL cholesterol $=$ Total-cholesterol - HDL-cholesterol $-(0.45 \times \text { triglycerides })^{133}$. S-LDL cholesterol was not calculated if S-triglycerides were $<0.2$ or $\geq 5 \mathrm{mmol} / 1$.

\subsubsection{Health related quality of life}

In paper I, two questionnaires were used to measure HRQL: The Medical Outcome Study 12 - item Short Form Health Survey (SF-12) ${ }^{134}$ and the Obesity and Weight-Loss Quality of life (OWLQOL-17) $)^{135,136}$. SF-12 is a commonly used generic measure of HRQL based on 12 questions or items from the larger 36-item Short Form Health Survey (SF-36) questionnaire. The items measure physical functioning, physical role limitations, bodily pain, general health, emotional role limitations, general health, emotional role limitations, vitality, social functioning and mental health. The items were scored in accordance with the recommendations from the scale author ${ }^{134}$ and then combined into two summary scores; the physical HRQL and the mental HRQL. The OWLQOL-17 $7^{135,136}$ is primarily a measure of the emotions and feelings ${ }^{137,138}$, which are believed to result from being obese and trying to lose weight. The instrument consists of 17 statements about weight-related feelings and emotions rated on a seven-point scale that ranges from 0 ("not at all") to 6 ("a very great deal"). The 17 items of the OWLOQOL form a scale ranging from 0 to 102 , with higher scores indicating a better emotional HRQL. 


\subsubsection{Physical activity}

In paper I, physical activity levels was investigated using a 7-day recall questionnaire validated against accelerometer in time spent engaged in physical activity of varying intensity in a normal weight population ${ }^{139}$. The questionnaire divides the day into five main parts: work, transport, domestic work, leisure time and sedentary activity, reporting time spent in physical activity in each of these parts. Physical activities were graded according to intensity by Ainsworth et $\mathrm{al}^{81}$, and time spent in physical activity $\geq 3$ METs and $\geq 5$ METs during the whole week were summed.

In Paper III, physical activity level was measured with a self-reported questionnaire $(\mathrm{CONOR})^{140}$ at 59 week follow-up and self-reported time spent in physical activity during an average week during the last year was registered. The CONOR questionnaire divides physical activity into two main parts: leisure time physical activity and occupational physical activity. Physical activity during leisure time was further divided into light physical activity (not sweaty and breathless) and vigorous physical activity (sweaty and breathless), and was scored according to type of work (mainly sedentary, mainly walking, mainly walking and carrying, heavy manual labor) ${ }^{140}$.

\subsubsection{Carotid to femoral (aortic) pulse wave velocity}

There are a number of different methods used in order to assess aortic arterial stiffness ${ }^{49}$, but cf PWV has emerged as the "gold standard" method because of its ease of use and its perceived reliability, and because of the large body of evidence across various populations demonstrating its association with cardiovascular disease independent of traditional risk factors $^{47,48,141-152}$. The Sphygmocor system (Atcor, Sidney, Australia) and a single highfidelity applanation tonometer (Millar $\left.{ }^{\circledR}\right)$ were used to measure ${ }_{\mathrm{cf}} \mathrm{PWV}$ in paper II and III. Pulse waves were obtained sequentially from the carotid and femoral artery. The locations of 
the sternal notch, carotid pulse and femoral pulse were located. The travel distance was calculated by subtracting the distance between the carotid artery and the sternal notch from the distance between the sternal notch and the femoral artery ${ }^{49}$. The subtracted travel distance measured at baseline was used in the calculations of ${ }_{\mathrm{cf}} \mathrm{PWV}$ at follow-up.

With patients lying in a horizontal position the ${ }_{\mathrm{cf}} \mathrm{PWV}$ was calculated from the transit time and the distance between carotid- and femoral artery sites, determined in relation to the Rwave of the ECG. Blood pressure was measured before every recording and entered into the Sphygmocor recorder in order to secure a valid recording with similar blood pressure. Three complete sets of data were collected and the average value was used as result.

\subsubsection{Bioelectrical impedance analysis}

The InBody 720 Body composition analyzer, Biospace Co. Ltd (Seol, South Korea) was used to measure body composition in paper II and III. The InBody 720 uses a multi-frequency electrical current at 5, 50, 250, 500 and 1000 kilohertz in order to measure the amount of extracellular and intracellular water. The InBody 720 uses segmental bioelectrical impedance analysis (BIA) in order to examine the body as five cylinders (four limbs and trunk), and measures impedance in these parts separately. Lean body mass consists of $73.3 \%$ water, regardless of race or gender ${ }^{153}$. When the volume of total body water is known then the lean body mass can be calculated. The amount of fat mass is obtained by subtracting the calculated lean body mass from total weight. The InBody 720 provides data on skeletal muscle mass, visceral fat area, fat mass and fat free mass. To avoid measurement error, measurements were conducted with patients in their underwear and positioned in an upright position on the body composition analyzer. All jewelry and wristwatches were removed before measurement. 


\subsubsection{Laboratory analysis}

In paper II and III analyses of serum glucose and blood lipids were performed using dry reagent slide technology on the Vitros FS 5.1 (Ortho-Clinical Diagnostics, New York, USA). Glycated hemoglobin (HbAlc) was analyzed using high performance liquid chromatography on Tosoh HLC-723 G7 (Tosoh Corporation, Tokyo, Japan). Sera for analysis of insulin were stored at $-20{ }^{\circ} \mathrm{C}$ and analyzed within one week of blood sampling (Linco Research Inc, St. Charles, MO).

\subsection{Sample size}

The sample size in paper I was calculated based on an estimated mean (standard deviation [SD]) weight loss of 4 (9) \% in the MLI group and 10 (9) \% in the ILI group. In order to achieve an $85 \%$ power $(\alpha=0.01)$ at least 120 individuals had to complete the study (60 in each group). The $\alpha$-value gives the probability of rejection of a null hypothesis that is actually true (type I error). To allow for a dropout rate of $40 \%$, we initially opted to include at least 200 patients (100 in each group).

The sample size in paper II and III was calculated based on Barinas-Mitchell et al's study ${ }^{154}$ which showed that a mean weight reduction of $8 \%$ resulted in $1.4 \mathrm{~m} / \mathrm{s}$ mean reduction in ${ }_{\text {cf }} \mathrm{PWV}$ in overweight patients with type 2 diabetes. From this, we hypothesized that the mean ${ }_{\text {cf }} \mathrm{PWV}$ would be $8.2 \mathrm{~m} / \mathrm{s}$ in both groups at baseline, and that a 1 -year weight loss of $8 \%$ in the ILI-group would lead to a mean (SD) of $1.4(2.8) \mathrm{m} / \mathrm{s}$ reduction in ${ }_{\mathrm{cf}} \mathrm{PWV}$. The expected weight loss following GBS is roughly 3 times as high, and we assumed that the mean reduction in ${ }_{\text {cf }} \mathrm{PWV}$ would be $2.8 \mathrm{~m} / \mathrm{s}$. Accordingly, at least 120 individuals had to complete the study in order to show a statistically significant difference between the two groups at a power of $80 \%(\alpha=0.05)$, To allow for a drop-out rate of $<40 \%$, we decided to include 200 patients within the study (100 in each group). 


\subsection{Statistical methods}

Data are presented as mean (SD) or number (\%) unless otherwise specified. Differences between groups at baseline were analyzed using independent samples t-tests for continuous data and Fisher's exact tests for categorical data. Unadjusted between groups differences were analyzed using independent samples t-test for continuous data and Fischer's exact test for categorical data. Within-groups changes from baseline to follow up were analyzed using paired samples t-tests for continuous data and McNemar's test for paired proportions. In paper I, we aimed to compare the effectiveness of an ILI and MLI in terms of changes in body weight, time spent in physical activity $>$ METS, time spent in physical activity $>$ MMETS, and physical-, mental- and emotional dimensions of HRQL as effect variables (dependents). The differences between groups were analyzed using analysis of covariance (ANCOVA) and include age, gender and the baseline value of the dependent variable as covariates $^{155}$. The main aim of the study in Paper I was to analyze the overall effect of the two treatment programs, and we thus performed an intention-to-treat analysis using ANCOVA with missing data imputed using multiple imputation (MI) ${ }^{156}$. MI involves three distinct steps. First, plausible values for the missing data are filled in $M$ times to generate $M$ complete datasets. Second, the $M$ complete datasets are analyzed using standard statistical methods. Thirdly, the results from the $M$ analyses are combined. Missing values at 1 year (weight, WC: $26 \%$, SF-36:47 \%, physical activity $>3$ and 5 METS: $48 \%$ and OWLQOL: $49 \%$ ) were imputed. The MI model consisted of the following predictor variables: treatment group, gender, age, baseline and 3 months weight and WC; while 1 year weight, WC and all physical activity measures and HRQL scores were used as both predictor and imputation variables. Through a fully conditional specification, applying linear regression as prediction method, we generated $M=5$ complete imputed datasets with 10 iterations per dataset. One year BMI (26 $\%$ missing) was calculated on the basis of measured height and the imputed weight variable. 
In order to test for clinically significant differences we calculated the effect size (ES) of the changes in HRQL ${ }^{157}$. Within-group ES was calculated as the mean HRQL change score between 1 year and baseline divided by the SD of HRQL at baseline in the respective group. Between-group ES was calculated as the difference in mean HRQL change score between groups at 1 year divided by the SD of baseline HRQL in both groups combined ${ }^{157,158}$. ES $<0.50$ was considered small, between 0.50 to 0.79 as moderate and $\mathrm{ES} \geq 0.80$ as large ${ }^{159}$. In paper II, we aimed to compare the effectiveness of an ILI and a LCD (paper II), while in paper III we aimed to compare the effectiveness of an ILI and a LCD followed by a Roux-en$\mathrm{Y}$ gastric bypass surgery. Both studies were performed with change in ${ }_{\mathrm{cf}} \mathrm{PWV}$ as the main effect variable (dependent).

The between-group differences in ${ }_{\mathrm{cf}} \mathrm{PWV}$ at follow up were assessed using ANCOVA and include age, gender, baseline BMI, baseline MAP, history of coronary artery disease and baseline value of ${ }_{\text {cf }} \mathrm{PWV}$ as covariates. The results are presented as mean $(95 \% \mathrm{CI})^{155}$.

\subsection{Ethics, approvals and founding}

All the study protocols (including revisions and amendments) in this thesis were approved by the regional ethics committee of the southern Norway Regional Health Authority (S-05715) (amendment to the original protocol "Overvektsprosjektet (OVP) i Helse Sør"). The studies were performed according to the principles expressed in the Declaration of Helsinki ${ }^{160}$, with written informed consent provided by all participants.

The study presented in paper I is registered in the ClinicalTrials.gov-registry with identifier NCT00464984.

The studies presented in paper II and III are registered in the ClinicalTrials.gov-registry with identifier NCT00626964.

This work has been funded in its entirety by Vestfold Hospital Trust. 


\section{Chapter 4. Results}

\subsection{Paper I}

Patients who accepted the invitation to participate and who satisfied the inclusion criteria were recruited consecutively from both centers to the ILI-group and MLI-group. The number of patients who declined to participate is unknown. A total of 372 patients were included, 232 (65\% female) in the ILI-group, and 140 (75\% female) in the MLI-group (Figure 8). There were no significant differences between groups in terms of age, body weight or physical activity level. However, the ILI-group had a lower proportion of women and higher mean (SD) scores in the mental dimension (48.0 (11.1) vs. 44.6 (11.8) and emotional dimension 42.9 (20.4) vs. $36.1(20.0)$ of HRQL, all $\mathrm{p}<0.04$.

Of the included patients in the ILI-group, a total of 225 (97\%) completed the first 12 weeks of the intervention and 181 (78 \%) attended the 1-year follow up. In the MLI-group a total of $131(94 \%)$ completed the intervention, but only 61 (44\%) attended the 1-year follow up (Figure 8). Completers in the ILI- and MLI-groups had an adherence rate of $86 \%$ (39 of 46 sessions) and $87 \%$ (13 of 15 sessions) respectively. 


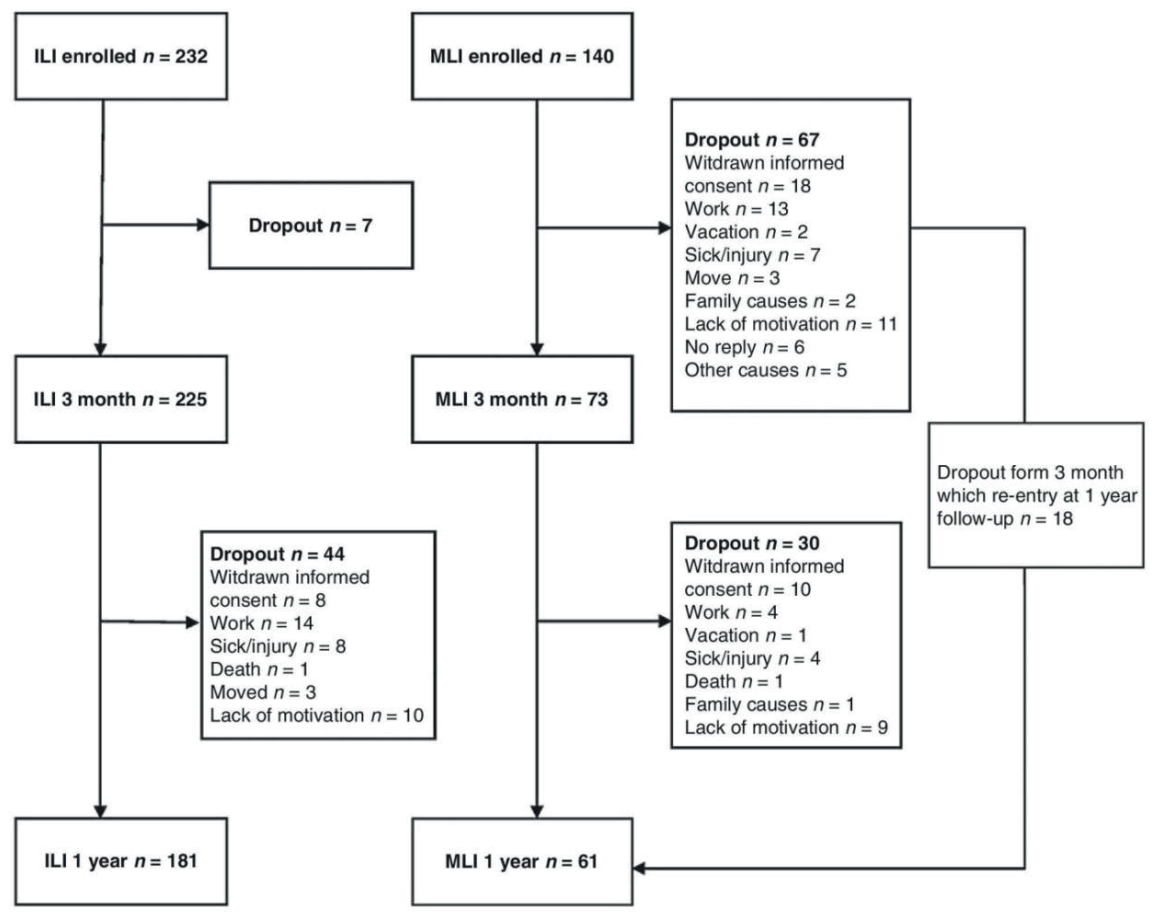

Figure 8. Flow chart of participants in paper I. No permission required ${ }^{124}$.

The mean $(95 \% \mathrm{CI}) 1$-year body weight reduction was significantly larger in the ILI group -10 $\%(-11,-9)$ compared to the MLI group $-2 \%(-6,-1), \mathrm{p}<0.001$ (figure 9). 


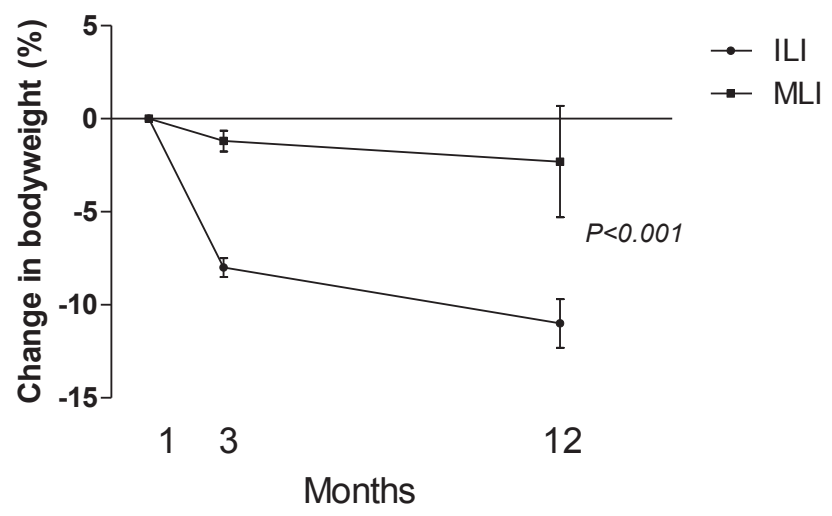

Figure 9. Mean body weight change (percent, 95\%CI) after 3 and 12 months of intensive lifestyle intervention (ILI) or moderate lifestyle intervention (MLI). Differences between groups at 1 year were assessed by ANCOVA, with age, gender and body weight at baseline as covariates.

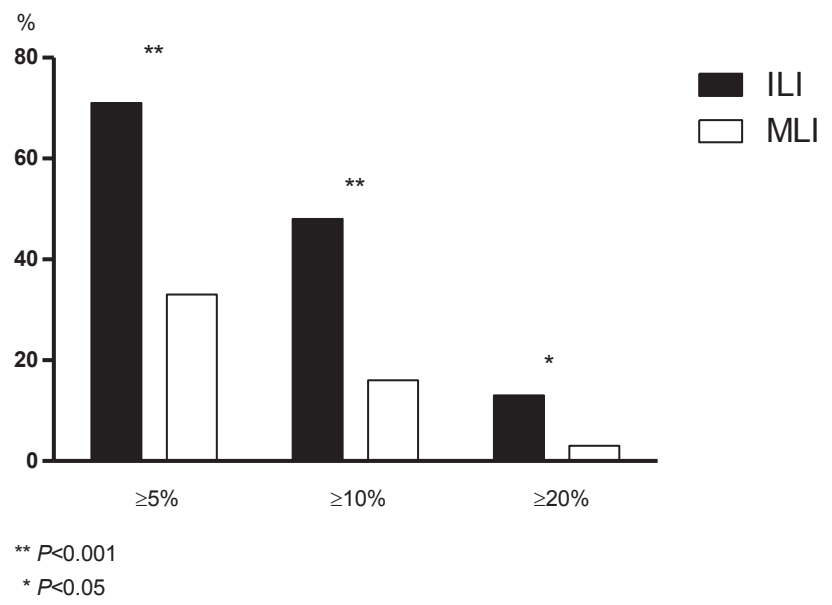

Figure 10. Percentage of all included patients who achieved weight loss $\geq 5 \%, \geq 10 \%$, and $\geq 20 \%$ after one year intensive lifestyle intervention (ILI) $n=225$ ) or moderate lifestyle intervention (MLI) (n=73). Between-group changes were analysed with Fisher's exact test. 
Further, the proportions of patients achieving $\geq 5 \%, \geq 10 \%$ or $\geq 20 \%$ weight loss were significantly higher in the ILI-group (figure 10).

There was no significant change in either levels of light- or moderate physical activity between patients in the ILI- or MLI group. Only patients in the ILI-group had a significantly increased level of light intensity physical activity (>3METs) of 29 (15 to 46) min and level of moderate intensity physical activity (>5METs) of 29 (21 to 39) at one year follow-up, both $\mathrm{p}<0.001$.

Patients in the ILI-group had significantly larger improvements in all dimensions of HRQL compared to patients in the MLI-group, and this was particularly pronounced in the emotional dimension with a between-group difference $(95 \% \mathrm{CI})$ of 17.8 (12.8 to 22.6$)$ points, $\mathrm{p}<0.001$, ES 0.77. Between-group differences in the physical dimension were 6.9 (4.4 to 9.3) points, $\mathrm{p}<0.001$, ES 0.54; and mental dimension 4.4 (1.4 to 7.4) points, $\mathrm{p}=0.018$, ES 0.18.

\subsection{Paper II \& III}

A total of 200 patients were included in the study (64\% female), 102 in the ILI-group and 98 in the LCD-group (Figure 11). The groups did not differ significantly in terms of ${ }_{\mathrm{cf}} \mathrm{PWV}$, but patients in the LCD-group were slightly younger and had higher systolic blood pressure and pulse pressure, higher body weight and fat mass, and lower prevalence of ischemic heart disease compared to patients in the ILI-group. During the first 7 weeks, 14 individuals in the ILI-group and 7 individuals in the GBS-group withdrew or did not attend the 7 week follow up. 


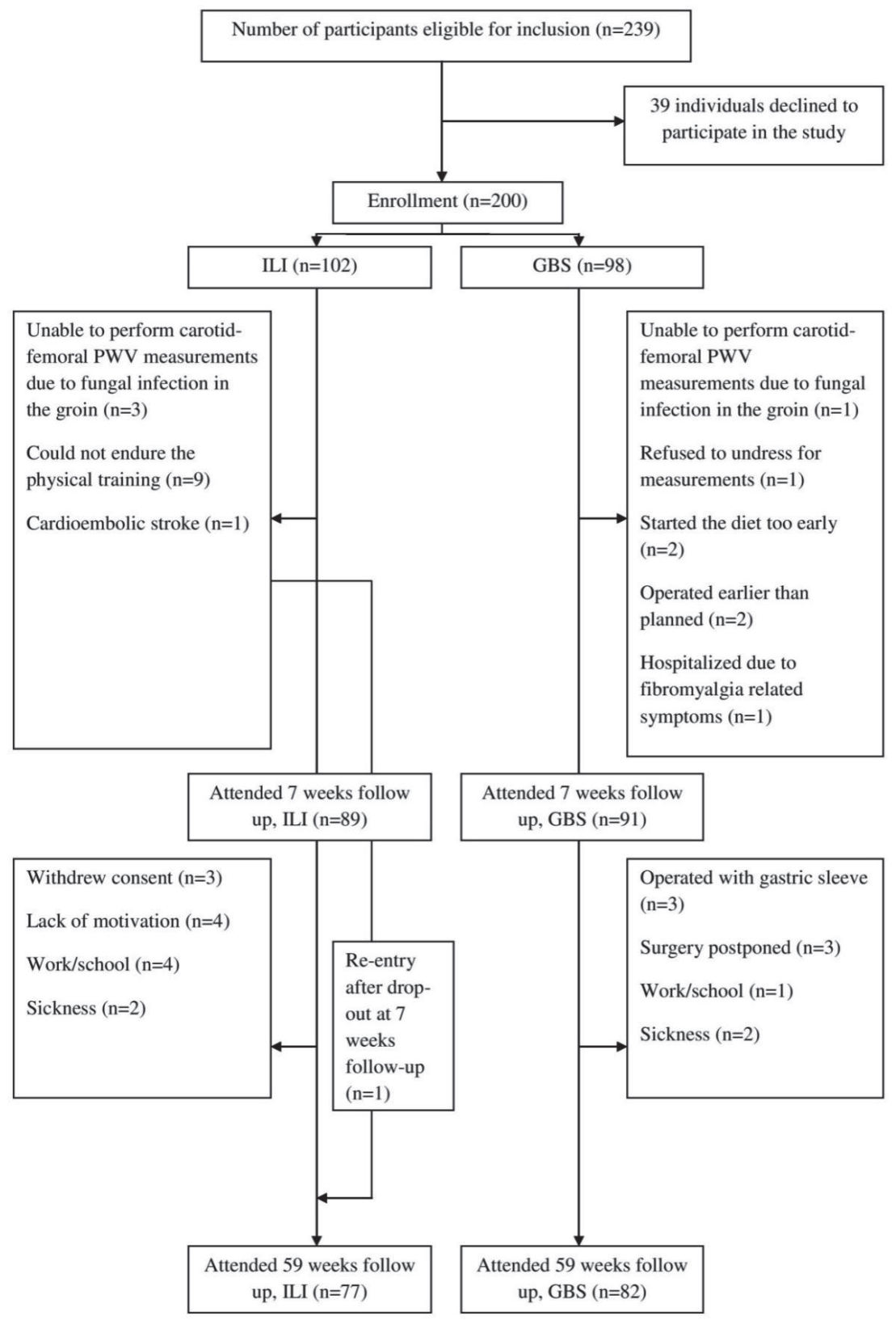

Figure 11. Flow chart of participants in paper II \& III. No permission required ${ }^{125}$. 


\subsubsection{Paper II}

After 7-weeks (paper II) the ILI-group had a larger reduction in ${ }_{\mathrm{cf}} \mathrm{PWV}$ compared to the LCDgroup, with an adjusted between-group mean difference $(95 \% \mathrm{CI})$ of $0.4(0.1$ to 0.6$) \mathrm{m} / \mathrm{s}$ (figure 12). Further adjustment for weight loss and change in MAP slightly attenuated the difference between groups, but it remained significant [0.3 ( 0.1 to 0.6$) \mathrm{m} / \mathrm{s}, \mathrm{p}=0.019]$. Only the ILI-group had a significant reduction in mean ${ }_{\mathrm{cf}} \mathrm{PWV}$, while the reduction in the LCDgroup was not statistically significant.

(a)

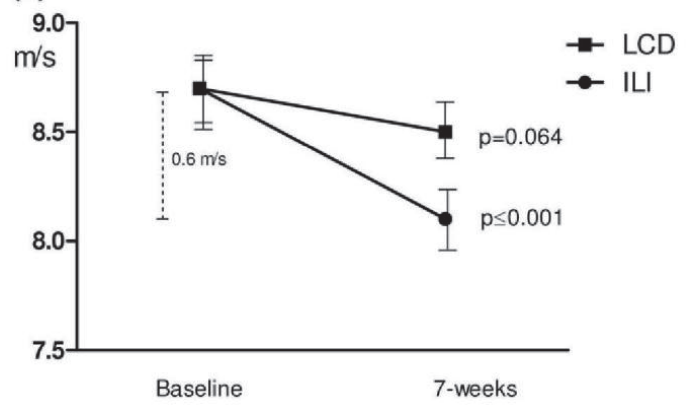

(b)

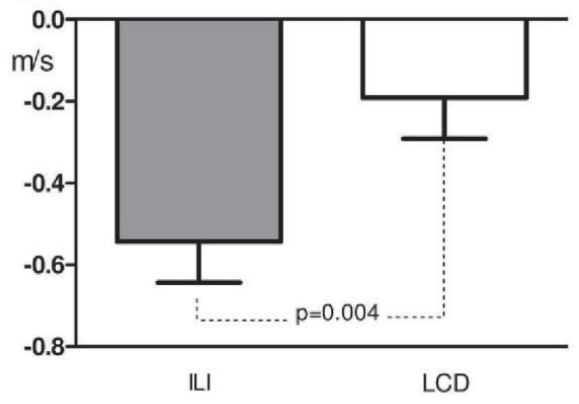

Figure 12. Changes in ${ }_{c f} \mathrm{PWV}$ after 7 weeks of follow-up. Within groups (a), adjusted difference between groups (b).

Both treatment groups demonstrated significant weight reductions, reduction in fat mass and skeletal muscle mass (all $\mathrm{p} \leq 0.001$ ), but adjusted analysis showed a significantly larger weight loss and reduction in skeletal muscle mass in the LCD-group; between groups difference of 1.5 ( 0.5 to 2.6$) \mathrm{kg}, \mathrm{p}=0.006$ and 0.8 ( 0.5 to 1.1$) \mathrm{kg}, \mathrm{p}<0.001$. No significant differences were apparent in terms of fat mass. Spearman's rank correlation analysis demonstrated no significant associations between change in ${ }_{c f} \mathrm{PWV}$ and weight loss $(\mathrm{r}=-0.02, \mathrm{p}=0.821)$, loss of skeletal muscle mass $(-0.06, \mathrm{p}=0.397)$ or loss of fat mass $(\mathrm{r}=0.04, \mathrm{p}=0.590)$. 


\subsubsection{Paper III}

During the final period of 52 weeks an additional 13 patients in the ILI-group and 9 patients in the GBS-group withdrew, while one patient reentered the ILI-group at 59 weeks follow-up. This leaves 159 (64\% female) completers ( $80 \%$ of all included patients) in the present analysis, a total number of 77 (76\%) and $82(84 \%)$ in the ILI-group and GBS-group, respectively (figure 11).

The adjusted analysis showed no significant differences in change of ${ }_{c f} \mathrm{PWV}$ between treatments groups $0.05(-0.4$ to 0.5$) \mathrm{m} / \mathrm{s}, \mathrm{p}=0.831$. Aortic ${ }_{\mathrm{cf}} \mathrm{PWV}$ did not change significantly from baseline to 59-weeks in either the ILI- or the GBS-group (figure 13).

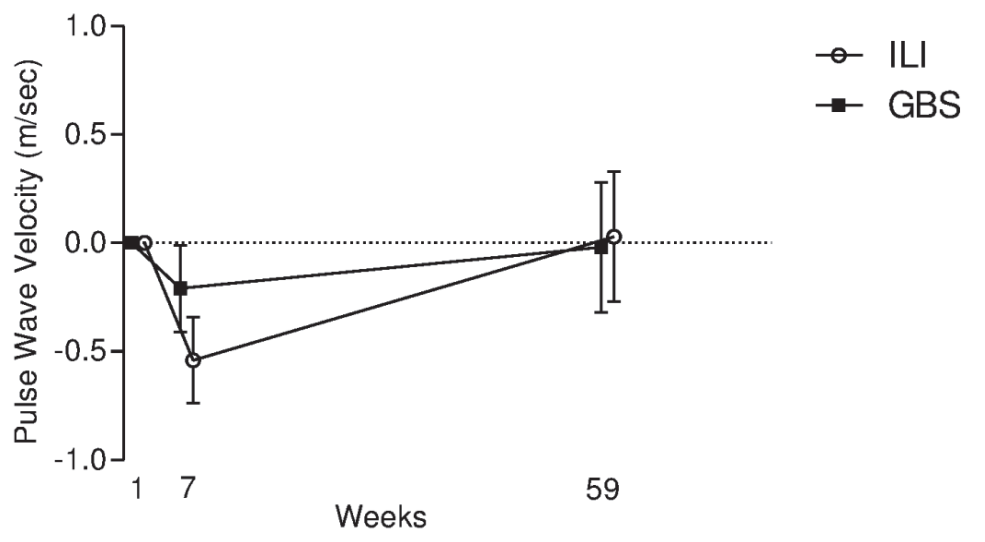

Figure 13. Short- and long-term mean adjusted changes in aortic carotid femoral pulse wave velocity $\left({ }_{\mathrm{cf}} \mathrm{PWV}\right)(\mathrm{m} / \mathrm{s}, 95 \% \mathrm{CI})$ in the morbidly obese patients who underwent intensive lifestyle intervention (ILI) or Roux-en-Y gastric bypass surgery (GBS). 

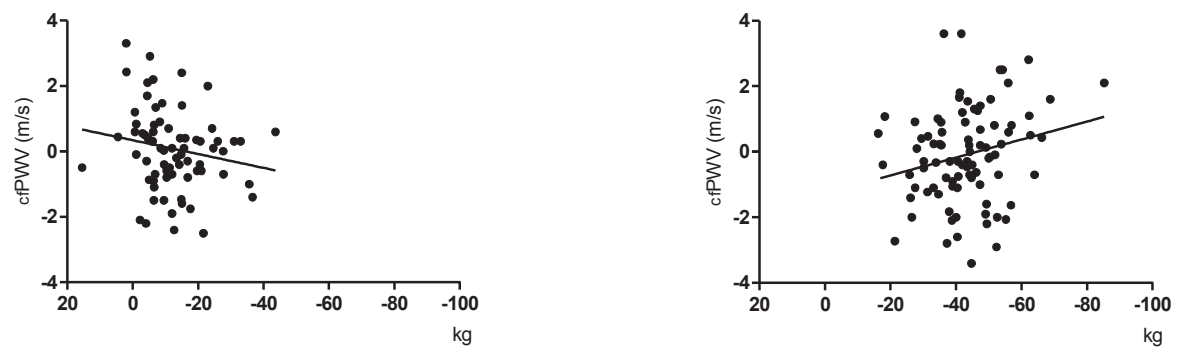

Weight loss
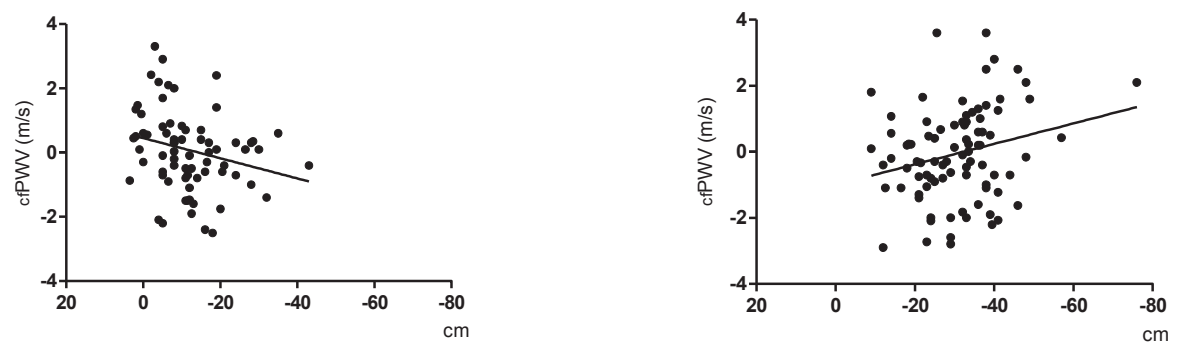

Waist circumference
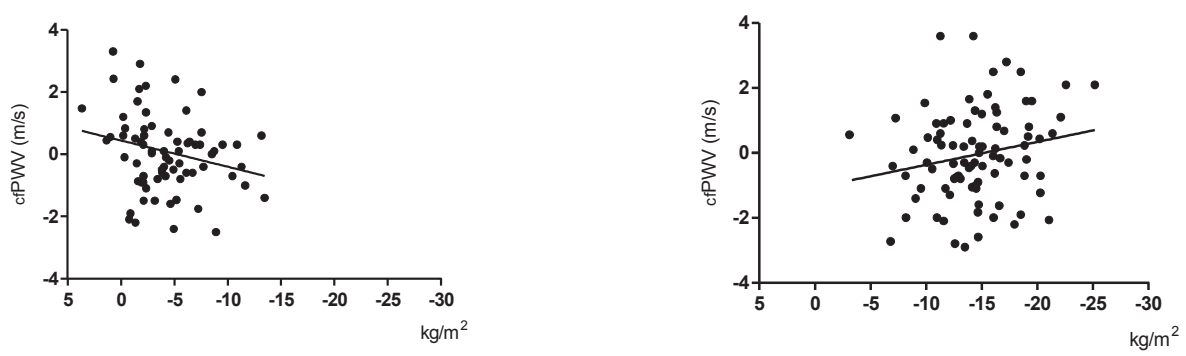

Body Mass Index

Figure 14. Scatterplots of the relationship between changes in the explanatory variables body weight, BMI and waist circumference, and changes in the effect variable ${ }_{\mathrm{cf}} \mathrm{PWV}$ within each treatment group. 
Reduction in body weight, BMI and waist circumference were associated with decreasing ${ }_{\text {cf }} \mathrm{PWV}$ in the ILI-group, but with increasing ${ }_{\text {cf }} \mathrm{PWV}$ in the GBS-group (figure 14). After adjustments for age, gender, history of coronary artery disease, and baseline values of BMI, MAP, heart rate and ${ }_{\mathrm{cf}} \mathrm{PWV}$, multiple linear regression analysis showed that the associations between changes in ${ }_{\mathrm{cf}} \mathrm{PWV}$ and changes in $\mathrm{WC}, \mathrm{BMI}$ and body weight remained statistically significant in the GBS-group only (all $\mathrm{p}<0.006)$.

There was a significantly larger reduction in heart rate in the GBS-group compared to the ILIgroup, with a between-group difference of 7 ( 3 to 10 ) beats/min, $p<0.001$. Both the ILI- and GBS-group had a significant reduction in heart rate of $-6(-8$ to -3$)$ beats $/ \mathrm{min}, \mathrm{p}<0.001$, and 11 ( -14 to -9$)$ beats $/ \mathrm{min}, \mathrm{p}<0.001$, respectively. There were significant reductions in the proportion of patients treated with anti-hypertensive and anti-diabetic drugs in the GBS-group only. Specifically, the number of patients treated with Renin-angiotensin-aldosterone-system (RAAS) -inhibitors, beta blockers and diuretics decreased from 23 to 13 (44\%), 10 to 4 (60\%), and 15 to 7 (53\%), all $p<0.031$. The use of sulfonylurea and metformin decreased from 8 to $0(100 \%)$ and 16 to $3(81 \%), p=0.008$ and $p<0.001$. There was no significant change in the use of statins in either group, both $p \geq 0.375$. The majority of patients reported that they completed $>3$ hours/week of light physical activity and $>3$ hours/week of vigorous physical activity at follow up, with no significant difference between groups (Table 3). 
Table 3. Self-reported physical activity at 59 weeks follow up in morbidly obese patients who completed the interventions.

\begin{tabular}{|c|c|c|c|c|}
\hline & & $\begin{array}{c}\text { Intensive lifestyle } \\
\text { intervention } \\
(\mathrm{n}=60)\end{array}$ & $\begin{array}{l}\text { Gastric bypass } \\
\text { surgery } \\
(n=54)\end{array}$ & $P$-value \\
\hline \multirow{4}{*}{$\begin{array}{l}\text { Light physical } \\
\text { activity }\end{array}$} & Nothing & $0(0)$ & $0(0)$ & 0.880 \\
\hline & $\leq 1$ hour/week & $1(2)$ & $1(2)$ & \\
\hline & 1-2 hours/week & $7(12)$ & $8(15)$ & \\
\hline & $\geq 3$ hours/week & $52(87)$ & $45(83)$ & \\
\hline \multirow{4}{*}{$\begin{array}{l}\text { Vigorous } \\
\text { physical activity }\end{array}$} & Nothing & $2(3)$ & $6(11)$ & 0.267 \\
\hline & $\leq 1$ hour/week & $5(8)$ & $7(13)$ & \\
\hline & 1-2 hours/week & $21(34)$ & $17(32)$ & \\
\hline & $\geq 3$ hours/week & $34(55)$ & $24(44)$ & \\
\hline \multirow[t]{5}{*}{ Work } & Sedentary work & $23(39)$ & $19(36)$ & 0.485 \\
\hline & Walking & $24(41)$ & $19(36)$ & \\
\hline & $\begin{array}{l}\text { Walking and } \\
\text { carrying }\end{array}$ & $11(19)$ & $11(21)$ & \\
\hline & Heavy manual & $1(2)$ & $4(8)$ & \\
\hline & labor & & & \\
\hline
\end{tabular}




\section{Chapter 5. Discussion}

The main results of the papers included in this thesis are:

Paper I) A 1-year intensive lifestyle intervention program at a public tertiary care centre was significantly more effective than a 1-year moderate lifestyle intervention program at a secondary care centre at producing weight loss and improving health related quality of life.

Paper II) Patients who underwent a comprehensive 7-week ILI program had a significantly larger reduction in aortic stiffness than those who completed a 7-week low calorie diet intervention.

Paper III) There were no significant differences between treatment groups and no changes in aortic stiffness after either intensive lifestyle intervention or Roux-en-Y gastric bypass surgery after 59 weeks, despite considerable weight loss in both groups.

The main limitations and strengths of the studies are summarized in Table 4 and discussed separately below. 
Table 4. Study design and the main limitations and strengths of papers I, II, and III.

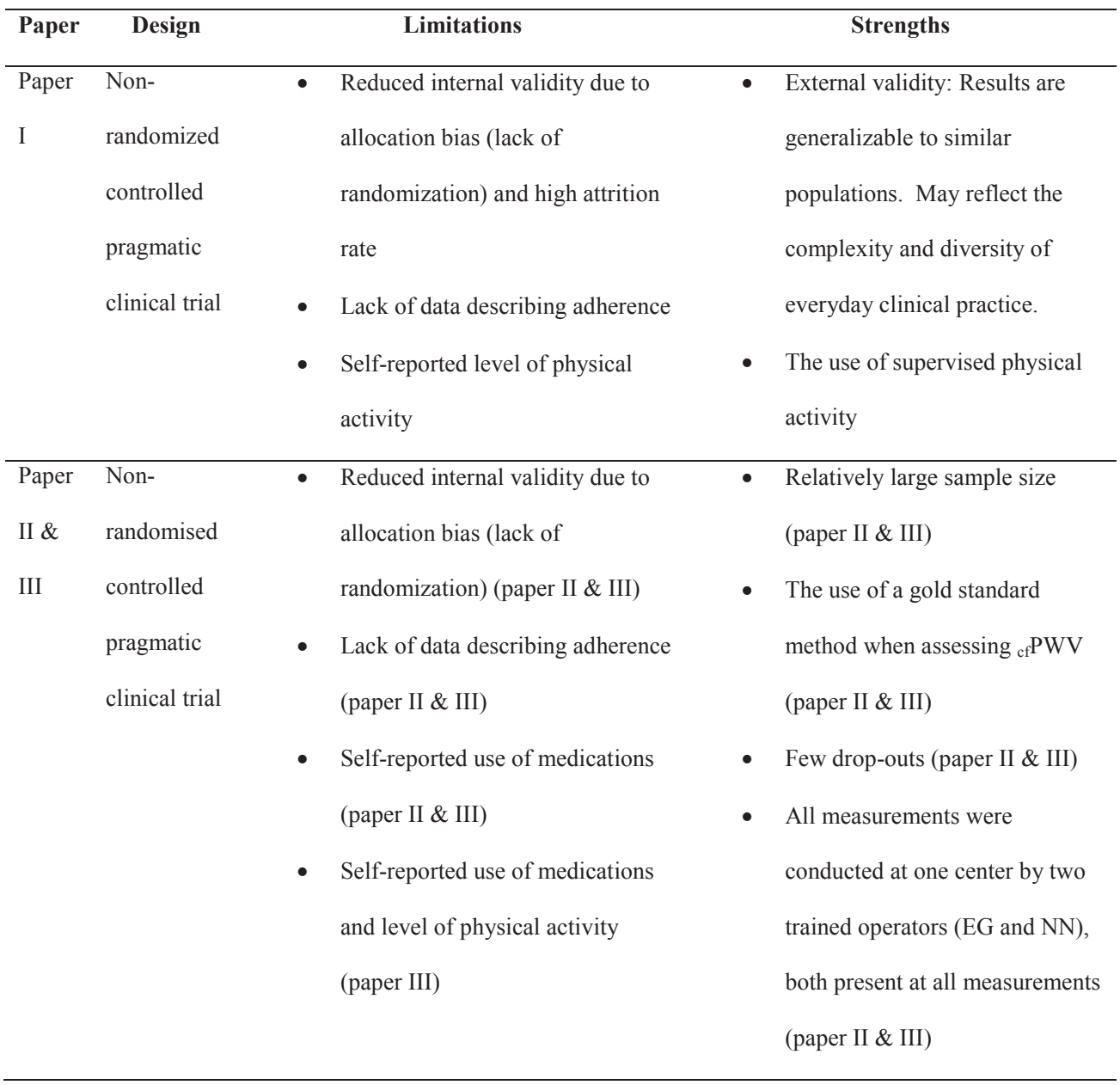

\subsection{Methodological considerations}

\subsubsection{Study design, generalizability and statistics}

\section{Study design}

In order to show the efficacy of various health care interventions it is recommended that randomized controlled trials are used ${ }^{161}$. Accordingly, it might be reasonable to question why 
the studies discussed in this thesis all have a non-randomised design. Although randomised trials often are preferable, randomisation is not always practicable or even desirable ${ }^{162}$. Weaknesses with a RCT-design could be several exclusion criteria, selection bias and low external validity, all with subsequent low generalisability ${ }^{163,164}$. In cases where there are good arguments against randomisation, useful clinical knowledge can be obtained through welldesigned non-randomized studies ${ }^{165}$. Since all treatments discussed in this thesis were publicly funded, patients were encouraged to take an active role in their choice of treatment ${ }^{79}$. We thus found it most appropriate to use a pragmatic non-randomized comparative study design. However, non-randomized trials are associated with lower internal validity and introduce elements of allocation and selection bias ${ }^{166}$. Patients included in the ILI-group in paper I were, for example, recruited from our tertiary health care center, while patients in the MLI-group were recruited from a local secondary care hospital, both of which treat only morbidly obese individuals. This referral of patients from different populations and locations introduced elements of selection bias. In addition, data on the number and characteristics of the patients who declined to participate in the study are missing. Treatment seeking morbidly obese patients in the southern part of Norway (Health Region South-East = Helse Sør-Øst) are primarily referred to secondary health care clinics from general practice, and, if needed, from secondary health care institutions to tertiary health care centers. Accordingly, patients recruited from tertiary health care centers probably have more severe forms of obesity and comorbidities than patients referred from secondary health care. Further, patients recruited from our tertiary health care center were probably more motivated for lifestyle intervention treatment, while the population referred from general practice consisted of both individuals motivated for lifestyle intervention and bariatric surgery. In addition, the ILI was more intensive and required greater absence from work and family compared to the MLI. This could have resulted in a higher proportion of highly motivated patients among patients in the 
ILI-group. This could also partly explain the difference in attrition rates between the two groups.

However, the main aim in the study presented in paper I was to compare the effectiveness of two outpatient lifestyle interventions offered treatment seeking morbidly obese individuals in southern Norway. While efficacy is used to assess if a treatment works, effectiveness relates to how well a treatment works in real life (normal clinical practice) ${ }^{123}$. Pragmatic trials are designed to better reflect the complexity and diversity of everyday clinical practice ${ }^{167}$, and makes it possible to compare and assess the effectiveness of two interventions relevant to real-life clinical care ${ }^{123,166}$. The results presented in Paper I may therefore contribute to the body of evidence from which decisions about the design and distribution of lifestyle interventions are based upon. The local obesity center has, as a consequence of this study, implemented changes in the MLI program to improve its effectiveness on various outcomes. The studies presented in paper II and III compared the effectiveness of two established treatment methods (lifestyle intervention with- or without surgery) on aortic arterial stiffness. However, these studies also have characteristics comparable with an efficacy trial design ${ }^{122}$, 123

We found it unethical to operate on individuals who both qualified for and preferred conservative treatment. As patients largely chose their treatment type themselves, this probably caused a selection of patients to the different treatments, e.g in terms of motivation for treatment (allocation bias). This thus limits the internal validity of the studies. In addition, the high attrition rate could cause attrition bias in all three studies, where this is most pronounced in paper I. It is plausible that patients who achieved a satisfactory weight loss or maintenance were more likely to attend follow up, while patients who did not achieve a satisfactory weight loss were more likely to drop out. This could be associated with larger 
differences in weight loss, and changes associated with weight loss (body composition, HRQL, physical activity level and aortic arterial stiffness) between treatment groups.

\section{Generalisability (external validity)}

All studies discussed in this thesis were performed in treatment seeking morbidly obese patients (from the southern part of Norway) who underwent various treatment programs offered by secondary and tertiary public health care centers. Accordingly, the results may be generalized to similar populations. However, the results may not be generalized to the general non-treatment seeking class II and III obese population which may differ from the participants in our studies with respect to comorbidities, socio-economics and other unknown factors. It is likely that the prevalence of obesity related comorbidities among patients with BMI between 35 and $40 \mathrm{~kg} / \mathrm{m}^{2}$ (class II) is higher in the sample population compared to the general class II obesity population. Further, in all three papers the included patients had to reside within $100 \mathrm{~km}$ from MOC or CPMR. During the period of patient inclusion in papers II and III, approximately 1500 patients were referred to and treated at MOC. In addition, the cohorts investigated in the papers consisted of mainly white Caucasians. This may limit the external validity of our results.

\section{Statistics}

We report two-tailed p-values and considered values below 0.05 to be significant. As the number of independent variables tested for significance in relation to an outcome increases, the risk of a significant result by chance increases (type 1 error - the incorrect rejection of a true null hypothesis) ${ }^{168}$. Since several statistical analyses were performed throughout the papers in this thesis, we cannot exclude the possibility that multiple comparisons might have biased our results, and particular attention should be directed towards results with small p- 
values e.g. $p<0.01$. One example of a $p$-value which should be interpreted with caution is the significant reduction in the emotional dimensions of HRQL in the MLI-group after adjustments $(\mathrm{p}=0.032)$. Although significant, one can ask if this represent a true effect in the MLI-group, since there was no significant reduction in either the mental or the physical dimension of HRQL.

In the study presented in paper I the main aim was to analyze the overall effect of the two treatment programs, not merely of completers, and we thus performed an intention-to-treat analysis using ANCOVA with missing data imputed using multiple imputation (MI) ${ }^{156}$. In MI the missing values are predicted using existing values from other variables. Standard statistical analyses were carried out on each imputed data set, producing multiple analyses results. These analyses results were then combined to produce one overall (pooled) result ${ }^{169}$, ${ }^{170}$. The missing values accounted for in Paper I may produce a risk of biased estimates, however the usage of multiple imputation most likely reduced this risk ${ }^{170}$. In paper I, a paired sample t-test showed that patients in the MLI-group did not experience significant weight loss after 1 year. On the other hand, after adjustments, according to the $95 \%$ CI the 1 year weight loss in both groups were statistically significant.

The clinical relevance of changes in HRQL was assessed by the calculation of effect size (ES) ${ }^{157,158}$. An ES ranging from 0.2 to 0.49 was considered small, an ES from 0.5 to 0.79 was considered moderate, and an ES greater than 0.8 was considered large ${ }^{159}$. Even if there are statistical within- or between group differences, the differences do not need to be clinically relevant. In paper I there were statistical significant differences between ILI and MLI in both the physical- $(\mathrm{p}<0.001)$, mental- $(\mathrm{p}=0.018)$ and emotional dimension of HRQL $(\mathrm{p}<0.001)$. However, the clinical relevance of these differences are considered low (mental HRQL $\mathrm{ES}=18$ ) to moderate (physical HRQL ES=0.54, emotional HRQL ES=0.77). 


\subsubsection{Hypothesis testing, limitations and validity of questionnaires}

\section{Paper I}

\section{Hypothesis testing}

The main hypothesis in paper I that "an ILI program (tertiary care level) would have a greater effectiveness than a MLI programme (secondary care level)" was confirmed in terms of weight loss and HRQL, but not in terms of physical activity level.

\section{Assessment of physical activity - self report questionnaire}

Physical activity level was measured using a 7-d recall questionnaire validated against accelerometer in time spent engaged in physical activity of varying intensity in a normal weight population ${ }^{139}$. This questionnaire divides the day into five main parts: work, transport, domestic work, leisure time and sedentary activity. Physical activities were graded according to intensity ${ }^{80,81}$, and time spent in physical activity $>3$ METs and $>5$ Mets during the whole week were summed, and average time per day spent in physical activity $>3$ METs and $>5$ METs were calculated. The value of this questionnaire in general, and especially in a morbidly obese population, is questionable. Self-reported physical activity measured with this questionnaire correlated moderately with physical activity measured with accelerometer in physical activities with intensities $<3$ METs $(r=0.47)$ and 3-6METs $(r=0.38)$, while there was a weak correlation measuring physical activities with intensity $>6$ METs $r=0.18)^{139}$. The questionnaire seems to underreport the amount of time spent in physical activity with intensity $>3$ METs and overreport the amount of time spent in physical activity with intensity ranging from 3-6 METs. However, this validation is performed in a normal-weight population. Further, there are several limitations and uncertainties associated with the use of self reported measurements of physical activity level in general, and obese individuals seem too 
considerably underreport energy intake and overreport the amount of physical activity undertaken ${ }^{171}$. On the other hand, we experienced that change in physical fitness could affect how patients assessed both the intensity and amount of physical activity undertaken. Individuals with higher degrees of physical fitness would most likely assess activities as less vigorous compared to less physically fit individuals. Patients in the ILI-group conducted a physical performance test on a motorized treadmill and demonstrated a significant increase in physical fitness from baseline (data not shown). This increase in physical fitness could thus cause an underreporting of their physical activity level. On the other hand, physically fit individuals might be more aware their physical activity level, which might lead to an overreporting of the amount of physical activity undertaken. The limitations and uncertainty associated with the use of this questionnaire to assess physical activity level weaken the validity of the data, and as a consequence interpretation of these data should be undertaken with caution.

\section{Assessment of Health Related Quality of Life - generic and disease specific questionnaires}

We used two questionnaires to measure HRQL: the Medical Outcome Study 12 - Item Short Form Health Survey (SF-12) ${ }^{134}$ and the Obesity and Weight-Loss Quality of Life (OWLQOL) $)^{135,136}$. The SF-36 is validated in patients with morbid obesity, and the two summary scales of the SF-36 demonstrate a satisfactory validity in morbidly obese patients ${ }^{172}$. Further, the results from SF-12 and SF-36 correlate strongly in obese and non-obese patients ${ }^{173}$. The OWLQOL has been validated in obese and morbidly obese individuals. The studies concluded that the instrument was, valid, reproducible and responsive in terms of the measurement of self-reported outcomes in obesity ${ }^{136}$. However, one limitation is that we did not include measurement of weight-related symptoms ${ }^{135}$, which might be of importance when comparing interventions with differences in amount-, type and intensity of activities. Due to 
this we were only able to investigate the mental, physical and emotional dimensions of HRQL.

\section{Paper II and III}

\section{Hypothesis testing}

The main hypothesis in paper II; that a seven-week ILI program consisting of aerobic endurance training 3 days a week and a moderate daily calorie restriction would have a greater effect on arterial stiffness than a seven-week LCD: was confirmed. In contrast, the main hypothesis in paper III: that patients who underwent Roux-en-Y gastric bypass surgery after an initial low calorie diet would have a larger mean 1-year reduction of ${ }_{\text {cf }} \mathrm{PWV}$ compared to those who underwent an intensive intervention program: was not confirmed.

Assessment of aortic pulse wave velocity $\left({ }_{c f} P W V\right)$

To measure ${ }_{\mathrm{cf}} \mathrm{PWV}$ we used the Sphygmocor system (Artcor, Sidney, Australia) and a single high-fidelity applanation tonometer $\left(\right.$ Millar $\left.^{\circledR}\right)$. The measurement of aortic arterial stiffness by ${ }_{\text {cf }} \mathrm{PWV}$ is considered to be the gold standard method ${ }^{49,146}$. Inaccuracies regarding measurements of distance could influence the absolute value of ${ }_{c f} \mathrm{PWV}$ and infer validity problems $^{174}$. In order to overcome this problem we placed the tape directly above the points of measurement with patients laying down, which in turn enabled us to get a valid measure of the carotid-to-femoral distance ${ }^{49}$. Due to bulging body parts in morbidly obese individuals, we cannot exclude that an error was introduced when the distances were measured. However, this possible bias has most likely not affected the results in Papers II and III given that carotid to femoral distance was measured only at baseline, and the only change between baseline and follow up was the time for the pulse wave to travel the distance measured at baseline ${ }^{175}$. 


\section{Weight loss and bioimpedance analysis}

Body composition was measured using bioimpedance analysis (BIA). BIA is not a gold standard method: it under predicts percentage body fat by a mean of $5.7 \%$ in men and $9.1 \%$ in women when compared with the hydrostatic weighing method ${ }^{176}$. The Inbody 720 used in papers II and III has not been validated against hydrostatic weighing, but it has been validated against Dual x-ray absorptiometry (DEXA) in obese patients, and has been shown to under predict percentage body fat in obese men by $5 \%$ and by $6 \%$ in women ${ }^{177}$. Further, the assumption of constant tissue hydration essential for BIA could be violated after weight loss. However, results from a study investigating the effect of one month of energy restriction in 48 obese subjects (mean weight loss $8.6 \mathrm{~kg}$ ) showed no vector displacement, indicating that liquid homeostasis was maintained after the weight loss ${ }^{178}$.

\section{Assessment of physical activity-the CONOR questionnaire}

To measure physical activity level in Paper III we used the CONOR (Cohort of Norway) questionnaire $^{140}$. The questions used in the CONOR questionnaire have been validated against accelerometer and $\mathrm{VO}_{2 \max }$ in normal weight men aged 20-39 years. There was a moderate agreement for hard leisure time physical activity with accelerometer (Spearman's $\rho=0.31$ ) and maximal oxygen consumption $\left(\mathrm{VO}_{2 \max }\right)(\rho=0.46)$, but no significant correlation in the case of light leisure time physical activity ${ }^{179}$. The instrument has also been validated against the long International Physical Activity Questionnaire (IPAQ-L), $\mathrm{VO}_{2 \max }$, anthropometric measurements and serum lipids in a multi-linguistic suburban population in Norway ${ }^{180}$. Hard leisure time physical activity showed weak, but consistent correlations with IPAQ-L, $\mathrm{VO}_{2 \max }$, anthropometric measurements and serum lipids in both men and women, but light physical activity showed consistent correlations only among women. There are problems associated with patient recollection and assessment of the amount of physical activity performed during 
the last year. This questionnaire has not been validated in obese individuals, and such individuals seem to overreport the amount of physical activity undertaken ${ }^{171}$. In the present study (paper III) most of the patients who completed the interventions (85\%) reported their highest light physical activity scores ( $\geq 3$ hours/week) in the final year of the study. $55 \%$ of completing patients reported the highest score ( $\geq 3$ hours/week) of vigorous physical activity. The questionnaire's apparent limitation as a means of assessing physical activity level may weaken the credibility of our results, and as a consequence interpretation of these data should be undertaken with caution.

\subsection{In the context of other studies}

\subsubsection{Paper I}

\section{Lifestyle intervention and body weight reduction}

Patients in the ILI-group achieved a 1 -year $11 \%$ weight loss. This is comparable with the results of previous lifestyle intervention studies $(6-16 \%)^{106-112,181-183}$. Further, $48 \%$ of the patients reached the treatment goal of $\geq 10 \%$ weight reduction from baseline, and $13 \%$ achieved $\geq 20 \%$ reduction. These results are inferior to those from a Norwegian study where $70 \%$ and $23 \%$ of the morbidly obese patients in an intermittent residential program achieved $\geq 10 \%$ and $\geq 20 \%$ reduction of body weight, respectively ${ }^{107}$. The intermittent residential program was more intensive in terms of duration, and patients in this study were heavier and had a larger percentage of men than our study, which may partly explain the difference. By contrast, a very large US-study (Look Ahead) of overweight and obese diabetic patients reported $38 \%$ of patients achieving a $\geq 10 \%$ reduction of bodyweight after ILI $^{111}$. This is lower than our results of $48 \%$ of patients achieving a $\geq 10 \%$ weight loss after 1 year. However, the intervention in this study was less intensive in terms of number of treatment 
days and the use of mainly non-supervised physical exercise. In addition, the population was a diabetic one and had a lower baseline body weight compared to the population in our study. The patients in the MLI-group achieved a $2 \%$ weight reduction, which might seem low compared with our treatment goal of a long-term weight loss of at least 5-10 \% ${ }^{77}$. However, this result is comparable with results from similar interventions ${ }^{110,111}$, but smaller than a $5 \%$ weight loss after a more intensive outpatient treatment for 6 months ${ }^{107}$. A proportion of $16 \%$ of patients in the MLI group achieved a weight loss $\geq 10 \%$, and $3 \%$ a weight loss $\geq 20 \%$, which is large compared to a similar intervention ${ }^{111}$. Compared with these results, our treatment goal of at least 5-10 \% weight loss after MLI seems to be somewhat optimistic.

\section{Lifestyle intervention and change in physical activity level}

Paper I shows that patients in the ILI-group increased the amount of time engaged in both physical activity above 3 and 5 METs, while there was no significant changes after the MLIprogram. These results indicate that more patients either increased or maintained their physical activity level, compared to the proportion which became less active. These trends are in accordance with the 1-year results of a partly residential ILI including Norwegian morbidly obese patients with a comparable weight loss ${ }^{109}$. However, the increase in amount of time engaged in physical activity in our study is in contrast to another Norwegian study measuring physical activity level with accelerometer in morbidly obese patients before and after an intermittent residential weight loss intervention. The study demonstrated a significant increase in time spent in moderate to vigorous physical activity after 4 weeks of intervention. But despite a comparable weight loss after 1 year, the study found no significant difference in physical activity level ${ }^{181}$. In our study we used a self reported questionnaire to measure physical activity, and one possible explanation for the different findings in terms of physical activity level might be related to the different methods used (accelerometry) to measure 
physical activity. However, a shorter intervention and smaller sample size than the ILI discussed in this thesis could also partly explain the difference.

\section{Lifestyle intervention and change in $H R Q L$}

In paper I, the MLI-group had lower scores on the mental- and emotional dimensions of HRQL at baseline. One might argue that the lower HRQL in the MLI-group might be explained by a higher proportion of women, which are known to have lower HRQL than men $^{66,184}$. However, post-hock analyses reveals that there were no significant differences between men and women in either the physical-, mental- or emotional dimensions of HRQL in either the ILI-group, MLI-group or both groups combined. 1 year post ILI we found significantly larger improvements in the physical-, mental- and emotional dimensions of HRQL than 1 year post MLI. The differences between groups were most pronounced in the emotional dimension, but were also significant in both the physical and mental dimensions. The results from the present study are concur partly with the results from a study comparing changes in HRQL after GBS and a partly residential ILI in morbidly obese patients in Norway ${ }^{68}$. In this study HRQL was measured using SF-36 and OWLQOL-17. The authors demonstrated a significant improvement in both the physical-, mental- and emotional dimensions, with ES $0.47,0.32$ and 0.67 respectively. The changes in the physical and emotional dimension were comparable, while the changes in the mental dimension were lower than in our study. Since the emotional dimension of HRQL was measured using the same instrument and the patients were comparable, the differences may partly be explained by differences in the interventions.

In accordance with our results, Faulconbridge et al. found improvement in both the physicaland mental dimensions of HRQL after 1 year of lifestyle modification in morbidly obese patients $^{185}$. Patients in the MLI-group did not achieve improvements in either physical or 
mental dimensions, but rather only on the emotional dimension of HRQL. This contrasts with a small randomized controlled trial of morbidly obese diabetic patients which compared changes in HRQL between GBS and a lifestyle intervention ${ }^{186}$. After 12 weekly group sessions with supervised group exercise and nutritional education, followed by monthly counseling for the reminding of the year, patients achieved improvement in both the physical and mental dimension of HRQL as measured by SF-36. This contradictory finding might possibly be explained by a more intensive lifestyle intervention, especially the final 9 months, in addition to differences in terms of the type of questionnaire. In addition, there were also differences between the trials in terms of age, gender distribution and bodyweight.

\subsubsection{Paper II \& III}

\section{Short - term study (paper II)}

The short-term results presented in paper II demonstrate a significant reduction in ${ }_{\mathrm{cf}} \mathrm{PWV}$ from baseline in the ILI-group only.

Our results contrast partly with the results from a short-term (12 weeks) randomized controlled study of overweight and obese healthy non-smoking middle-aged volunteers, which showed that a hypocaloric diet intervention (1200-1500 kcal/day) with no emphasis on physical activity was associated with a $7 \mathrm{~kg}$ weight reduction and a significant reduction in

${ }_{\text {cf }} \mathrm{PWV}$ of $1.3 \mathrm{~m} / \mathrm{s}^{187}$. Despite a comparable weight loss, there was a considerably larger effect on ${ }_{\text {cf }} \mathrm{PWV}$ compared to our study. However, in contrast to this healthy obese population, the participants in our study were heavier and the majority had obesity related comorbidities. The results from the study in Paper II indicate that a weight loss of $9 \mathrm{~kg}$ in the LCD group is insufficient to significantly reduce ${ }_{\mathrm{cf}} \mathrm{PWV}$ in morbidly obese individuals.

In the view of these results, we wanted in paper II to explore the determinants of changes in ${ }_{\text {cf }} \mathrm{PWV}$. Post-hoc, we constructed a predictive linear regression model of 7 weeks change in 
${ }_{\text {cf }} \mathrm{PWV}$ (dependent) from appropriate baseline variables (independents) commonly associated with ${ }_{\mathrm{cf}} \mathrm{PWV}$ changes: gender, age, history of coronary artery disease, type 2 diabetes, smoking, baseline MAP, baseline BMI and baseline ${ }_{\mathrm{cf}} \mathrm{PWV}$. Directed acyclic graphs ${ }^{188}$ and baseline differences between groups were used as tools to identify the variables to be included in this model. After backward selection of variables in the multivariate model, only gender and baseline ${ }_{\mathrm{cf}} \mathrm{PWV}$ were retained. We subsequently performed a second multiple linear regression analysis with 7 weeks change in ${ }_{\mathrm{cf}} \mathrm{PWV}$ as dependent variable, and forced treatment-group into the model together with the remaining independent variables (from the backward model), namely gender and baseline ${ }_{c f} \mathrm{PWV}$, using the enter option. According to the second multiple regression model, only baseline ${ }_{\mathrm{cf}} \mathrm{PWV}$ and treatment group had independent significant effects on 7 weeks change in ${ }_{\text {cf }} \mathrm{PWV}$. Squared part correlation coefficients showed that treatment group and baseline ${ }_{\text {cf }} \mathrm{PWV}$ explained $2 \%$ and $24 \%$ of the 7 weeks change in ${ }_{\mathrm{cf}} \mathrm{PWV}$, respectively (table 5).

Table 5. Linear multiple regression analysis using 7-weeks change in ${ }_{\mathrm{cf}} \mathrm{PWV}$ as dependent variable.

\begin{tabular}{lccc}
\hline Variable & Std. $\beta$-coefficients & Part correlation & p-value \\
& & squared & \\
\hline Gender & 0.139 & 0.02 & 0.055 \\
Baseline ${ }_{\text {cf }} \mathrm{PWV}(\mathrm{m} / \mathrm{s})$ & -0.513 & 0.24 & $<0.001$ \\
Treatment group & 0.149 & 0,02 & 0.033 \\
\hline
\end{tabular}

\section{Lifestyle Intervention and effects on ${ }_{c f} P W V$}

Previous studies have demonstrated beneficial short-term (7-24 weeks) effects of various lifestyle intervention programs on ${ }_{\mathrm{cf}} \mathrm{PWV}$ in normal weight ${ }^{189}$, overweight ${ }^{190}$ and obese 
populations ${ }^{187,191}$. The favorable short-term effects of ILI on ${ }_{\mathrm{cf}} \mathrm{PWV}$ in the present study are in accordance with the results from a randomised controlled study of overweight and obese healthy normotensive non-diabetic, which showed an average weight reduction of $-7 \mathrm{~kg}(8 \%)$ and a significant reduction in ${ }_{\mathrm{cf}} \mathrm{PWV}$ of $-0.5 \mathrm{~m} / \mathrm{s}$ after 6 months. In contrast to our results in paper III, this significant reduction was maintained after 12 months ${ }^{192}$. The ILI in this study consisted of a diet and physical activity intervention, with- or without sodium reduction ${ }^{191}$. However, compared to our study the ILI in study referred to above was less intensive in terms of treatment days, but had a longer time period from baseline to short-term follow-up. Further, the population in this study was, when compared to our own population group, younger, had a lesser degree of obesity, had a smaller proportion of male individuals and had less obesity related comorbidities, compared to the population in our study. It is likely that individuals with obesity related comorbidities, like the majority of our patients, have a higher frequency of structural changes associated with aortic stiffness in the aortic wall compared to metabolic healthy obese ${ }^{193,194}$, and a potential reversal of these changes may take several years ${ }^{195}$.

\section{Long term study (paper III)}

The long-term results in paper III showed that the favorable short-term effect of ILI on ${ }_{\mathrm{cf}} \mathrm{PWV}$ was temporary, and there was no significant change from baseline to 59 weeks follow up, despite a considerable weight loss of $12 \mathrm{~kg}(10 \%)$ from baseline. These results are in contrast to a randomised controlled study of 38 obese individuals with type 2 diabetes but otherwise healthy, which evaluated the effect of a behavioral weight loss intervention program combined with either orlistat or placebo. The intervention included an energy restriction of approximately $500 \mathrm{kcal}$ per day and the participants were encouraged to undertake 40-60 min of moderate intensity physical activity most weekdays. Independent of orlistat the participants showed an average weight reduction of $10 \mathrm{~kg}(8 \%)$ and a reduction of ${ }_{\mathrm{cf}} \mathrm{PWV}$ of $0.5 \mathrm{~m} / \mathrm{s}$ after 
1 year of ILI $^{154}$. However, compared to our study this population consisted of individuals with a lesser degree of obesity at baseline and a lesser degree of obesity related comorbidities (except from type 2 diabetes). Our long-term results did not confirm the results from a study of 49 healthy overweight to severe obese individuals comparing the effect of a low fat diet and low carbohydrate diet in terms of weight loss and reduction of ${ }_{\mathrm{cf}} \mathrm{PWV}$. The low carbohydrate diet group demonstrated a slightly larger weight reduction $-14.9 \mathrm{~kg}$ vs $-11.5 \mathrm{~kg}$, but both groups achieved a significant reduction in ${ }_{\mathrm{cf}} \mathrm{PWV}$ of $1.4 \mathrm{~m} / \mathrm{s}$ vs $1.5 \mathrm{~m} / \mathrm{s}$, no significant changes between groups ${ }^{196}$. However, direct comparison between these studies is difficult given the differences in various degrees of obesity and the frequency of obesity related comorbidities.

\section{Bariatric surgery and effect on ${ }_{c f} P W V$}

After GBS, the individuals in the GBS-group in the arterial stiffness study achieved a weight loss of $43 \mathrm{~kg}(32 \%)$ from baseline. To our surprise, there was no significant change in ${ }_{\mathrm{cf}} \mathrm{PWV}$ after 59 weeks despite this considerable weight loss. However, a significant number of individuals in the GBS-group stopped taking anti-hypertensive and anti-diabetic medications during the study. The reduction of antihypertensive medications might partly explain the small increase in blood pressure and the absence of beneficial changes in aortic stiffness in the GBS-group, as ${ }_{\mathrm{cf}} \mathrm{PWV}$ is partly pressure dependent. Anti-hypertensive medications have the potential to reduce aortic stiffness beyond the blood lowering effect ${ }^{197}$. A reduction in the use of metformin might also have worked against a weight loss induced decline in ${ }_{\mathrm{cf}} \mathrm{PWV}^{198}$. Our results contrast with those from a study of obese healthy adults which aimed to investigate the effect of weight loss on aortic PWV, demonstrating a beneficial long-term effect on PWV after bariatric surgery ${ }^{199}$. However, in the aforementioned study aortic PWV was measured at the ascending-, descending aorta and the aortic arch. In addition, 
measurements were performed using magnetic resonance imaging -the use of different locations and methods to measure aortic PWV makes it difficult to compare. In contrast to this obese but healthy population, the population in our study consisted of treatment seeking morbidly individuals, with a majority having obesity related comorbidities.

\section{Impact of weight loss on changes in ${ }_{c f} P W V$ according to treatment group}

The lack of a reduction in ${ }_{\mathrm{cf}} \mathrm{PWV}$ despite a considerable weight loss in both groups was somewhat surprising. However, subgroup analyses demonstrated that weight loss and reduced WC were associated with long-term decreasing ${ }_{c f} \mathrm{PWV}$ in the ILI-group. By contrast, and to some surprise, weight loss was associated with increasing ${ }_{\mathrm{cf}} \mathrm{PWV}$ in the GBS-group (figure 14 , results). In order to explore the impact of ILI and GBS on ${ }_{c f} \mathrm{PWV}$ further we performed a multiple linear regression analyses with long-term ${ }_{c f} \mathrm{PWV}$-changes as dependent variable, and age, gender, history of coronary artery disease, and baseline values of BMI, MAP, heart rate, ${ }_{\text {cf }} \mathrm{PWV}$ and anthropometric parameters as explanatory variables. The regression analyses did not reveal any significant association between weight loss in terms of reduction in body weight, BMI and waist circumference. This is in accordance with the negative findings after lifestyle intervention in the SAVE trial ${ }^{191}$. However, the negative associations between changes in ${ }_{\mathrm{cf}} \mathrm{PWV}$ (dependent) and changes in WC, BMI and body weight remained statistically significant (all $\mathrm{p}<0.006)$ in the GBS-group. This association was somewhat surprising and difficult to explain. Given the well known limitations of post-hoc subgroup analyses, this finding should, however, be interpreted with caution ${ }^{200}$. One idea might be that the massive weight loss in the GBS-group may lead to a reflex increase in arterial resistance as the hyporesponsive baroreflex caused by obesity related chronic vasodilatation is abolished $^{201}$. 


\section{Possible mechanisms for changes in arterial stiffness}

Aortic arterial stiffness is influenced by both functional and structural factors ${ }^{193,194,202,203}$. Three primary elements of the arterial wall are considered to determine arterial stiffness: first, the proportion of collagen and elastin; second, fractures of elastic lamella and the crosslinking of collagen and advanced glycation end-products; and third, the vasoconstriction exerted by smooth muscle cells ${ }^{193,194}$. The first two elements are considered structural, with changes occurring over a period of years ${ }^{195}$, and will contribute to early vascular aging $(\text { EVA })^{204-206}$. By contrast, vasoconstriction by vascular smooth muscle is a functional change that may occur very rapidly. In addition, other factors like adrenerg activity and aerobic exercise may modulate aortic compliance within a short space of time ${ }^{193,194}$. Heart rate, blood pressure, change in muscle mass and insulin resistance may also affect aortic arterial stiffness, and these changes occur over a short period of time and well within the timeframe of the interventions in paper II. Further, one might speculate that the lack of change in ${ }_{\mathrm{cf}} \mathrm{PWV}$ after 59 weeks despite a considerable weight loss might be explained by the more slowly occurring structural changes over a longer period of time.

\section{Heart rate}

Heart rate affects arterial mechanics, but has a greater effect on elastic arteries than muscular $\operatorname{arteries}^{207}$. The effect of heart rate might possibly be explained by the viscoelastic properties of the arterial wall, as there is an increased arterial stiffness as a result of an increased rate of stretching and vice versa ${ }^{50}$. A post-hoc analysis on the short-term results of the arterial stiffness study showed that the mean $(95 \% \mathrm{CI})$ reduction in heart rate was larger in the ILIgroup after adjustment for age, gender and baseline heart rate, $5.2(2,8)$ beats/min, $p=0.020$. However, the reduction in heart rate had no significant effect in the fully adjusted ANCOVA model, $\mathrm{p}=0.123$. In contrast, the long-term results showed that the participants in the GBS- 
group had the largest reduction of heart rate $11.7(10,14)$ beats/min, $\mathrm{p}<0.001$. A post-hoc analyses showed that the reduction in heart rate had a small but significant effect in the fully adjusted ANCOVA model and explained $5 \%$ of the variance in ${ }_{\text {cf }} \mathrm{PWV}$ after 59 weeks (Partial Eta Squared 0.047, $\mathrm{p}=0.008$ ).

\section{Blood pressure}

Blood pressure affects aortic PWV. The short term results (paper II) show a reduction of systolic blood pressure in both the ILI- and LCD-group, but only the ILI-group achieved a significant reduction in diastolic blood pressure. In contrast, the long term results (paper III) showed that there was no significant change in blood pressure in the ILI-group, and only a significant reduction in systolic blood pressure in the GBS-group. The blood pressure variable that best reflects aortic stiffness is not systolic or diastolic blood pressure, but rather pulse pressure $^{208,209}$. A post-hoc analysis showed that neither change in mean arterial pressure nor change in pulse pressure after 7 weeks had a significant effect on ${ }_{\mathrm{cf}} \mathrm{PWV}$ in the fully adjusted ANCOVA model, $\mathrm{p}=0.364$, and $\mathrm{p}=0.900$, respectively. However, after 59 weeks both change in pulse pressure and change in MAP had a small but significant effect on ${ }_{\mathrm{cf}} \mathrm{PWV}$ in the fully adjusted ANCOVA model Partial Eta Squared 0.104, p $<0.001$ and Partial Eta Squared 0.168, $\mathrm{p}<0.001$, respectively. After 59 weeks change in pulse pressure and change in MAP explained $10 \%$ and $17 \%$ of the variance in ${ }_{c f} \mathrm{PWV}$, respectively.

\section{Change in muscle mass and insulin resistance}

Age related reduction in muscle mass is associated with increased arterial stiffness ${ }^{210,211}$. Loss in muscle mass can reduce insulin sensitivity ${ }^{212}$, which contributes to a increase in arterial stiffness independent of blood pressure ${ }^{213}$. One of the main mechanisms involved here is the formation of advanced glycation end-product on the arterial wall, leading to cross-linking of 
collagen molecules which in turn may lead to loss of collagen elasticity and a subsequent increase in arterial stiffness ${ }^{214}$.

After 7 weeks there was a significant loss of muscle mass among individual in both groups, but significant larger loss after LCD, $\mathrm{p} \leq 0.001$. However, after 59 weeks there was only a significant loss of muscle mass among individuals in the GBS-group, and also a significant between groups difference, $p<0.001$. There were no significant differences between groups in terms of insulin sensitivity after 7 weeks. However, after 59 weeks there were significant reductions in terms of insulin, glucose, $\mathrm{HbA1C}$ and HOMA-IR in both groups, but the reductions were more pronounced among individuals in the GBS-group, all $\mathrm{p} \leq 0.002$. Accordingly, the loss of muscle mass, especially in the GBS-group, has the potential to explain some of the absence of reduction of ${ }_{\mathrm{cf}} \mathrm{PWV}$ after 59 weeks. However, the significant reduction in insulin, glucose, HbA1C and HOMA-IR suggest the effect of loss of muscle mass on ${ }_{\mathrm{cf}} \mathrm{PWV}$ to be limited.

\section{Physical activity}

Several studies indicate that physical activity has a direct effect on arterial compliance

independent of changes in body weight, blood pressure, lipids and heart rate ${ }^{215-217}$. However, the mechanisms behind this are not completely understood. Mechanisms induced by exercise might be: deformation of the vessel which may combat collagen cross-linking, vascular smooth muscle cells relaxation $^{216}$. Several mechanisms may contribute to vascular smooth muscle cell relaxation, such as increased arterial wall sheared stress and bioactivity of nitric oxide, and reductions in vasoconstrictor tone, oxidative stress and inflammation ${ }^{217,218}$. The short-term changes after aerobic exercise may suggest that these changes are of a more functional than structural character. Our short term results indicate that the difference in reduction of ${ }_{\mathrm{cf}} \mathrm{PWV}$ between the treatment groups was largely independent of weight loss, 
insulin sensitivity, heart rate, blood pressure and lipids. One might speculate that the betweengroup difference in change in ${ }_{\mathrm{cf}} \mathrm{PWV}$ is related to physical activity, which is a main difference between the two interventions. The physical activity level in an average week over the course of the last year was measured with a self reported questionnaire. According to these results there were no significant differences in either light physical activity, vigorous physical activity or occupational physical activity. Even though we do not have any measures of changes in physical activity levels in either of the groups, we know that during the first 12 weeks of the intervention patients participated in 6 supervised exercise sessions each week, which contrasts with those individuals in the LCD-group. One explanation might be that the physical activity level among participants in the ILI-group decreased, contributing to the lack of a reduction in ${ }_{\mathrm{cf}} \mathrm{PWV}$ among patients in the ILI-group. In order to investigate the impact of physical activity and physical fitness on arterial stiffness, long-term studies with objective measurement of physical activity level (accelerometry) and physical fitness (indirect calorimetry) are needed.

\section{Change in use of anti-diabetic and anti-hypertensive drugs}

As was discussed earlier, ${ }_{\text {cf }} \mathrm{PWV}$ is partly pressure dependent, with a change in antihypertensive medication having the potential to affect ${ }_{\mathrm{cf}} \mathrm{PWV}$. There were no significant differences in usage of anti-hypertensive or anti-diabetic medication between groups at baseline, and during the first 7 week one individual in each group stopped using hypertensive medications. In the same period there were no changes in use of anti-diabetic medications among individuals in the ILI-group, while there were 2 who stopped using Glitazone, 5 who stopped using sylfonylurea and 1 who stopped using metformin. At 59 week follow-up there was a significant reduction in patients treated with anti-hypertensive or anti-diabetic drugs among patients in the surgery group. Specifically, the use of RAAS-inhibitors, beta-blockers, 
diuretics, sulfonylurea and metformin reduced significantly, all $\mathrm{p}<0.032$. There were no significant changes in statins in either group. As anti-hypertensive medications have the potential to reduce aortic stiffness beyond the blood pressure lowering effect ${ }^{197}$, the reduction in usage of anti-hypertensive medications might explain some of the small increases in blood pressure and the absence of beneficial changes in ${ }_{\mathrm{cf}} \mathrm{PWV}$ seen in the GBS-group despite their substantial weight loss. Further, the use of metformin ${ }^{198,219}$ and atorvastatin ${ }^{220}$ have been associated with reduced arterial stiffness. The discontinuation of metformin might also have mitigated any weight loss induced decline in ${ }_{\text {cf }} \mathrm{PWV}$ in the GBS-group. 


\section{Chapter 6. Clinical implications}

\section{Paper I}

In paper I we demonstrate that a combination of moderate energy restriction and supervised physical activity (ILI) was superior to a less intensive lifestyle intervention in terms of 1-year weight loss and health related quality of life in treatment seeking morbidly obese patients. According to our results, the MLI-programme, as performed in present study, may be insufficient in itself to induce meaningful weight loss in morbidly obese patients. Accordingly, the local obesity center has implemented changes in the MLI programme in order to improve it`s results.

\section{Paper II}

Even though energy restriction is one of the cornerstones of obesity treatment and has many positive metabolic effects, the short-term reduction of cardiovascular risk is likely to be greater when energy restriction is combined with physical activity. We demonstrate that in spite of a slightly lower 7-week weight loss and comparable effects on blood pressure, insulin sensitivity and lipids, patients in the ILI-group achieved a significantly larger reduction in ${ }_{\text {cf }} \mathrm{PWV}$ compared to patients in the LCD-group. These results indicate that in treatment seeking morbidly obese individuals a combination of caloric restriction and supervised physical exercise might be a more effective combatant of aortic arterial stiffness than isolated caloric restriction. Whether this beneficial effect transforms into lower cardiovascular morbidity and mortality is unknown. 


\section{Paper III}

In paper III, we show that the short-term effects demonstrated in paper II were of a temporary character, and one may speculate as to whether these changes were caused by adaptations of the functional elements of the aorta. In order to achieve persistent adaptations of the functional elements of the aorta caused by for instance physical exercise, it is likely that the exercise needs to be maintained. In addition, some of the long-term effects of ${ }_{c f} \mathrm{PWV}$ are likely to be the results of changes in structural elements of the aorta, such as changes in the collagen-elastin ratio, fractures of elastic lamella and the cross-linking of collagen. These are changes that are modified over a period of years. In order to give any definitive recommendation, further verification and follow up for several years is needed. 


\section{Chapter 7. Summary}

\section{Paper I}

We compared the effectiveness of an intensive lifestyle intervention programme (ILI) at a tertiary care centre and a moderate lifestyle intervention programme (MLI) conducted at a secondary care centre in morbidly obese individuals. The ILI was associated with a significantly larger weight reduction and greater improved physical, mental and emotional dimensions of health related quality of life than the MLI.

\section{Paper II}

We compared the 7-week effect of a low calorie diet and an ILI program on aortic arterial stiffness in morbidly obese individuals. Individuals in the ILI-group achieved a significantly greater reduction in ${ }_{\mathrm{cf}} \mathrm{PWV}$ compared to the LCD-group, indicating that short-term moderate caloric restriction (minus $1000 \mathrm{kcal} /$ day) combined with supervised physical exercise might be superior to an isolated more pronounced caloric restriction (Low calorie diet $<900$ kcal/day) among Norwegian treatment seeking morbidly obese individuals.

\section{Paper III}

We compared the 1-year effect of an ILI-program and a surgical weight loss program on ${ }_{\text {cf }} \mathrm{PWV}$ in morbidly obese individuals. There were no significant changes in ${ }_{\mathrm{cf}} \mathrm{PWV}$ after either ILI or GBS after 59 weeks, despite a large weight loss in both groups. Our findings indicate that weight loss, either induced by GBS or ILI, has no significant long-term effect on aortic stiffness in Norwegian treatment-seeking morbidly obese individuals. 


\section{Chapter 8. Topics for future research}

Long -term effectiveness of intensive lifestyle intervention

The one year effect of intensive lifestyle intervention in terms of changes in body weight, HRQL and physical activity level needs to be confirmed in well designed and carefully conducted randomised controlled trials and observational studies (e.g. comparative effectiveness research) with long-term follow up.

Measurement of physical activity and physical fitness.

Further studies of physical activity in morbidly obese individuals should use accelerometry in order to achieve an objective measurement of physical activity level.

Aerobic exercise and aortic PWV

Paper II demonstrated a significant reduction of aortic PWV after 7 weeks of lifestyle intervention consisting of aerobic exercise and caloric restriction. Paper III showed that these favorable changes were of temporary character. One might argue that these favorable changes in aortic PWV might be sustained if the physical activity level, in terms of intensity, frequency and durations were maintained. One future topic is to address the functional adaptations to sustained physical activity level and the possible effect on aortic PWV in morbidly obese individuals.

Weight loss and long-term changes in aortic PWV.

Paper III demonstrated that there were no significant long-term effects on aortic PWV after either ILI or GBS, despite considerable weight loss in both groups. A major part of the longterm changes in aortic stiffness are probably caused by structural changes and develop over 
years, and these structural changes might develop beyond the timeframe of the studies presented in this thesis. In order to investigate the impact of weight loss on the structural changes in the aortic wall long term studies are required. 


\section{References}

1. The Norwegian Directorate of Health. Norwegian patients rights act.

2004.http://helsedirektoratet.no/publikasjoner/lov-om-pasientrettigheter/Publikasjoner/lov-ompasientrettigheter.pdf

2. The Ministry of Health Care Services. Treatment of morbid obesity in adults. 2005. http://www.helse-midt.no/upload/77388/page.html

3. The Ministry of Health Care Services. Utredning og behandling av sykelig overvekt $\mathrm{i}$ spesialisthelsetjenesten - Voksne. 2007

http://www.nordlandssykehuset.no/getfile.php/NLSH bilde\%20og\%20filarkiv/Medisinsk\%20klinikk/r apport\%20utredning\%20og\%20beah\%20av\%20sykelig\%20overvekt\%20spesialist\%20helsetjenesten. pdf.

4. Renjilian DA, Perri MG, Nezu AM, McKelvey WF, Shermer RL and Anton SD. Individual versus group therapy for obesity: effects of matching participants to their treatment preferences. $J$ Consult Clin Psychol. 2001; 69: 717-21.

5. Sturm R. Increases in morbid obesity in the USA: 2000-2005. Public Health. 2007; 121: 492-6.

6. Wang YC, McPherson K, Marsh T, Gortmaker SL and Brown M. Health and economic burden of the projected obesity trends in the USA and the UK. Lancet. 2011; 378: 815-25.

7. Buchwald H and Oien DM. Metabolic/bariatric surgery worldwide 2011. Obes Surg. 2013; 23: 427-36.

8. Mala T and Kristinsson J. [Acute internal hernia following gastric bypass for morbid obesity]. Tidsskr Nor Laegeforen. 2013; 133: 640-4.

9. Midthjell $\mathrm{K}$, Lee CMY, Langhammer A, et al. Trends in overweight and obesity over 22 years in a large adult population: the HUNT Study, Norway. Clinical Obesity. 2013;3: 12-22.

10. Obesity: preventing and managing the global epidemic. Report of a WHO consultation. World Health Organ Tech Rep Ser. 2000; 894: i-xii, 1-253.

11. Physical status: the use and interpretation of anthropometry. Report of a WHO Expert Committee. World Health Organ Tech Rep Ser. 1995; 854: 1-452.

12. Scott HW, Jr. and Law DHt. Clinical appraisal of jejunoileal shunt in patients with morbid obesity. Am J Surg. 1969; 117: 246-53.

13. Hubbard VS and Hall WH. Gastrointestinal Surgery for Severe Obesity. Obes Surg. 1991; 1: 257-65.

14. Buchwald H. Consensus conference statement bariatric surgery for morbid obesity: health implications for patients, health professionals, and third-party payers. Surgery for obesity and related diseases : official journal of the American Society for Bariatric Surgery. 2005; 1: 371-81.

15. Swinburn BA, Sacks G, Hall KD, et al. The global obesity pandemic: shaped by global drivers and local environments. Lancet. 2011; 378: 804-14.

16. Finucane MM, Stevens GA, Cowan MJ, et al. National, regional, and global trends in bodymass index since 1980: systematic analysis of health examination surveys and epidemiological studies with 960 country-years and 9.1 million participants. Lancet. 2011; 377: 557-67.

17. $\mathrm{Ng} \mathrm{M}$, Fleming $\mathrm{T}$, Robinson $\mathrm{M}$, et al. Global, regional, and national prevalence of overweight and obesity in children and adults during 1980-2013: a systematic analysis for the Global Burden of Disease Study 2013. Lancet. 2014.

18. Flegal KM, Carroll MD, Kit BK and Ogden CL. Prevalence of obesity and trends in the distribution of body mass index among US adults, 1999-2010. JAMA. 2012; 307: 491-7.

19. Freedman DS, Khan LK, Serdula MK, Galuska DA and Dietz WH. Trends and correlates of class 3 obesity in the United States from 1990 through 2000. JAMA. 2002; 288: 1758-61.

20. Norwegian Institute of Public Health - Utvikling av fedme i Norge 1960 - 2000. 2004.

21. World Health Organization - Global health risks: mortality and burden of disease attributable to selected major risks. 2009. 
22. Engeland A, Bjorge $\mathrm{T}$, Selmer RM and Tverdal A. Height and body mass index in relation to total mortality. Epidemiology. 2003; 14: 293-9.

23. Calle EE, Thun MJ, Petrelli JM, Rodriguez $\mathrm{C}$ and Heath CW, Jr. Body-mass index and mortality in a prospective cohort of U.S. adults. N Engl J Med. 1999; 341: 1097-105.

24. Fontaine KR, Redden DT, Wang C, Westfall AO and Allison DB. Years of life lost due to obesity. JAMA. 2003; 289: 187-93.

25. Flegal KM, Graubard BI, Williamson DF and Gail MH. Excess deaths associated with underweight, overweight, and obesity. JAMA. 2005; 293: 1861-7.

26. Whitlock $G$, Lewington $S$, Sherliker $P$, et al. Body-mass index and cause-specific mortality in 900000 adults: collaborative analyses of 57 prospective studies. Lancet. 2009; 373: 1083-96.

27. Haheim LL, Tonstad S, Hjermann I, Leren P and Holme I. Predictiveness of body mass index for fatal coronary heart disease in men according to length of follow-up: a 21-year prospective cohort study. Scandinavian journal of public health. 2007; 35: 4-10.

28. Jousilahti P, Tuomilehto J, Vartiainen E, Pekkanen J and Puska P. Body weight, cardiovascular risk factors, and coronary mortality. 15-year follow-up of middle-aged men and women in eastern Finland. Circulation. 1996; 93: 1372-9.

29. Hubert HB, Feinleib M, McNamara PM and Castelli WP. Obesity as an independent risk factor for cardiovascular disease: a 26-year follow-up of participants in the Framingham Heart Study. Circulation. 1983; 67: 968-77.

30. Thomsen M and Nordestgaard BG. Myocardial Infarction and Ischemic Heart Disease in Overweight and Obesity With and Without Metabolic Syndrome. JAMA internal medicine. 2013.

31. Katzmarzyk PT, Church TS, Janssen I, Ross R and Blair SN. Metabolic syndrome, obesity, and mortality: impact of cardiorespiratory fitness. Diabetes Care. 2005; 28: 391-7.

32. Golubic R, Ekelund U, Wijndaele K, et al. Rate of weight gain predicts change in physical activity levels: a longitudinal analysis of the EPIC-Norfolk cohort. Int J Obes (Lond). 2013; 37: 404-9.

33. Renehan AG, Tyson M, Egger M, Heller RF and Zwahlen M. Body-mass index and incidence of cancer: a systematic review and meta-analysis of prospective observational studies. Lancet. 2008; 371: 569-78.

34. Subak LL, Wing R, West DS, et al. Weight loss to treat urinary incontinence in overweight and obese women. N Engl J Med. 2009; 360: 481-90.

35. Jacobson BC, Somers SC, Fuchs CS, Kelly CP and Camargo CA. Body-Mass Index and Symptoms of Gastroesophageal Reflux in Women. N Engl J Med. 2006; 354: 2340-8.

36. Young T, Palta M, Dempsey J, Skatrud J, Weber S and Badr S. The occurrence of sleepdisordered breathing among middle-aged adults. N Engl J Med. 1993; 328: 1230-5.

37. Felson DT, Anderson JJ, Naimark A, Walker AM and Meenan RF. Obesity and knee osteoarthritis. The Framingham Study. Ann Intern Med. 1988; 109: 18-24.

38. Zhao G, Ford ES, Dhingra S, Li C, Strine TW and Mokdad AH. Depression and anxiety among US adults: associations with body mass index. Int J Obes (Lond). 2009; 33: 257-66.

39. Daviglus ML, Liu K, Yan LL, et al. Body mass index in middle age and health-related quality of life in older age: the Chicago heart association detection project in industry study. Arch Intern Med. 2003; 163: 2448-55.

40. Rider OJ, Francis JM, Ali MK, et al. Beneficial cardiovascular effects of bariatric surgical and dietary weight loss in obesity. J Am Coll Cardiol. 2009; 54: 718-26.

41. McAuley PA and Blair SN. Obesity paradoxes. J Sports Sci. 2011; 29: 773-82.

42. Tackling obesity in England. Health Educ Res. 2001; 16: 399-400.

43. Allison DB, Fontaine KR, Manson JE, Stevens J and Vanltallie TB. Annual deaths attributable to obesity in the United States. JAMA. 1999; 282: 1530-8.

44. Lim SS, Vos T, Flaxman AD, et al. A comparative risk assessment of burden of disease and injury attributable to 67 risk factors and risk factor clusters in 21 regions, 1990-2010: a systematic analysis for the Global Burden of Disease Study 2010. Lancet. 2012; 380: 2224-60.

45. Livingston $\mathrm{EH}$ and Ko $\mathrm{CY}$. Effect of diabetes and hypertension on obesity-related mortality. Surgery. 2005; 137: 16-25. 
46. Ben-Shlomo Y, Spears M, Boustred C, et al. Aortic pulse wave velocity improves cardiovascular event prediction: an individual participant meta-analysis of prospective observational data from 17,635 subjects. J Am Coll Cardiol. 2014; 63: 636-40.

47. Cruickshank K, Riste L, Anderson SG, Wright JS, Dunn G and Gosling RG. Aortic pulse-wave velocity and its relationship to mortality in diabetes and glucose intolerance: an integrated index of vascular function? Circulation. 2002; 106: 2085-90.

48. Vlachopoulos C, Aznaouridis K and Stefanadis C. Prediction of cardiovascular events and allcause mortality with arterial stiffness: a systematic review and meta-analysis. J Am Coll Cardiol. 2010; 55: 1318-27.

49. Laurent S, Cockcroft J, Van Bortel L, et al. Expert consensus document on arterial stiffness: methodological issues and clinical applications. Eur Heart J. 2006; 27: 2588-605.

50. Nichols W.W ORMF. McDonald's blood flow in arteries: theoretical, experimental and clinical principles 4th ed. 1998.

51. Toto-Moukouo JJ, Achimastos A, Asmar RG, Hugues CJ and Safar ME. Pulse wave velocity in patients with obesity and hypertension. Am Heart J. 1986; 112: 136-40.

52. Wildman RP, Mackey RH, Bostom A, Thompson T and Sutton-Tyrrell K. Measures of obesity are associated with vascular stiffness in young and older adults. Hypertension. 2003; 42: 468-73.

53. Caspersen CJ, Powell KE and Christenson GM. Physical activity, exercise, and physical fitness: definitions and distinctions for health-related research. Public Health Rep. 1985; 100: 126-31.

54. Juneau CE and Potvin L. Trends in leisure-, transport-, and work-related physical activity in Canada 1994-2005. Prev Med. 2010; 51: 384-6.

55. Brownson RC, Boehmer TK and Luke DA. Declining rates of physical activity in the United States: what are the contributors? Annu Rev Public Health. 2005; 26: 421-43.

56. Petersen CB, Thygesen LC, Helge JW, Gronbaek M and Tolstrup JS. Time trends in physical activity in leisure time in the Danish population from 1987 to 2005 . Scandinavian journal of public health. 2010; 38: 121-8.

57. Stamatakis E, Ekelund $U$ and Wareham NJ. Temporal trends in physical activity in England: the Health Survey for England 1991 to 2004. Prev Med. 2007; 45: 416-23.

58. Blair SN and Brodney S. Effects of physical inactivity and obesity on morbidity and mortality: current evidence and research issues. Med Sci Sports Exerc. 1999; 31: S646-62.

59. van der Ploeg HP, Chey T, Korda RJ, Banks E and Bauman A. Sitting time and all-cause mortality risk in 222497 Australian adults. Arch Intern Med. 2012; 172: 494-500.

60. Wareham NJ, van Sluijs EM and Ekelund U. Physical activity and obesity prevention: a review of the current evidence. Proc Nutr Soc. 2005; 64: 229-47.

61. Hays RD, Anderson R and Revicki D. Psychometric considerations in evaluating health-related quality of life measures. Qual Life Res. 1993; 2: 441-9.

62. Fontaine KR, Barofsky I and Cheskin L. Predictors of quality of life for obese persons. J Nerv Ment Dis. 1997; 185: 120-2.

63. Fontaine KR and Barofsky I. Obesity and health-related quality of life. Obesity reviews : an official journal of the International Association for the Study of Obesity. 2001; 2: 173-82.

64. Kolotkin RL, Meter K and Williams GR. Quality of life and obesity. Obesity reviews : an official journal of the International Association for the Study of Obesity. 2001; 2: 219-29.

65. Larsson U, Karlsson J and Sullivan M. Impact of overweight and obesity on health-related quality of life--a Swedish population study. Int J Obes Relat Metab Disord. 2002; 26: 417-24.

66. Duval K, Marceau P, Lescelleur O, et al. Health-related quality of life in morbid obesity. Obes Surg. 2006; 16: 574-9.

67. Kolotkin RL, Crosby RD, Williams GR, Hartley GG and Nicol S. The relationship between health-related quality of life and weight loss. Obes Res. 2001; 9: 564-71.

68. Karlsen T, Lund R, Roislien J, et al. Health related quality of life after gastric bypass or intensive lifestyle intervention: a controlled clinical study. Health and quality of life outcomes. 2013; 11: 17. 
69. Aftab H, Risstad H, Sovik TT, et al. Five-year outcome after gastric bypass for morbid obesity in a Norwegian cohort. Surgery for obesity and related diseases : official journal of the American Society for Bariatric Surgery. 2014; 10: 71-8.

70. Warkentin LM, Das D, Majumdar SR, Johnson JA and Padwal RS. The effect of weight loss on health-related quality of life: systematic review and meta-analysis of randomized trials. Obesity reviews : an official journal of the International Association for the Study of Obesity. 2014; 15: 169-82. 71. Jepsen R, Aadland E, Andersen JR and Natvig GK. Associations between physical activity and quality of life outcomes in adults with severe obesity: a cross-sectional study prior to the beginning of a lifestyle intervention. Health and quality of life outcomes. 2013; 11: 187.

72. Martin CK, Church TS, Thompson AM, Earnest CP and Blair SN. Exercise dose and quality of life: a randomized controlled trial. Arch Intern Med. 2009; 169: 269-78.

73. Imayama I, Alfano CM, Kong A, et al. Dietary weight loss and exercise interventions effects on quality of life in overweight/obese postmenopausal women: a randomized controlled trial. The international journal of behavioral nutrition and physical activity. 2011; 8: 118.

74. Obesity: The Prevention, Identification, Assessment and Management of Overweight and Obesity in Adults and Children. London: National Institute for Health and Clinical Excellence., 2006. 75. Jensen MD, Ryan DH, Apovian CM, et al. 2013 AHA/ACC/TOS Guideline for the Management of Overweight and Obesity in Adults: A Report of the American College of Cardiology/American Heart Association Task Force on Practice Guidelines and The Obesity Society. J Am Coll Cardiol. 2014; 63: 2985-3023.

76. Clinical Guidelines on the Identification, Evaluation, and Treatment of Overweight and Obesity in Adults--The Evidence Report. National Institutes of Health. Obes Res. 1998; 6 Suppl 2: 51S209S.

77. The Ministry of Health Care Services. National professional guidelines for prevention, diagnosis and treatment of overweight and obesity in adults.

2011.http://www.helsedirektoratet.no/publikasjoner/nasjonal-faglig-retningslinje-for-forebyggingutredning-og-behandling-av-overvekt-og-fedme-hos-voksne/Publikasjoner/nasjonal-fagligretningslinje-for-forebygging-utredning-og-behandling-av-overvekt-og-fedme-hos

78. Logue J, Thompson L, Romanes F, Wilson DC, Thompson J and Sattar N. Management of obesity: summary of SIGN guideline. BMJ. 2010; 340: c154.

79. Jakobsen GS, Hofso D, Roislien J, Sandbu R and Hjelmesaeth J. Morbidly obese patients--who undergoes bariatric surgery? Obes Surg. 2010; 20: 1142-8.

80. Ainsworth BE, Haskell WL, Leon AS, et al. Compendium of physical activities: classification of energy costs of human physical activities. Med Sci Sports Exerc. 1993; 25: 71-80.

81. Ainsworth BE, Haskell WL, Herrmann SD, et al. 2011 Compendium of Physical Activities: a second update of codes and MET values. Med Sci Sports Exerc. 2011; 43: 1575-81.

82. Lee CD, Blair SN and Jackson AS. Cardiorespiratory fitness, body composition, and all-cause and cardiovascular disease mortality in men. Am J Clin Nutr. 1999; 69: 373-80.

83. Wei M, Kampert JB, Barlow CE, et al. Relationship between low cardiorespiratory fitness and mortality in normal-weight, overweight, and obese men. JAMA. 1999; 282: 1547-53.

84. Fogelholm M. Physical activity, fitness and fatness: relations to mortality, morbidity and disease risk factors. A systematic review. Obesity reviews : an official journal of the International Association for the Study of Obesity. 2010; 11: 202-21.

85. Jakicic JM, Marcus BH, Gallagher KI, Napolitano M and Lang W. Effect of exercise duration and intensity on weight loss in overweight, sedentary women: a randomized trial. JAMA. 2003; 290: 1323-30.

86. Jakicic JM. The effect of physical activity on body weight. Obesity (Silver Spring, Md). 2009; 17 Suppl 3: S34-8.

87. Curioni CC and Lourenco PM. Long-term weight loss after diet and exercise: a systematic review. Int J Obes (Lond). 2005; 29: 1168-74.

88. Garrow JS and Summerbell CD. Meta-analysis: effect of exercise, with or without dieting, on the body composition of overweight subjects. Eur J Clin Nutr. 1995; 49: 1-10. 
89. Pate RR, Pratt M, Blair SN, et al. Physical activity and public health. A recommendation from the Centers for Disease Control and Prevention and the American College of Sports Medicine. JAMA. 1995; 273: 402-7.

90. Jakicic JM and Davis KK. Obesity and physical activity. Psychiatr Clin North Am. 2011; 34: 829-

40.

91. Mekary RA, Feskanich D, Hu FB, Willett WC and Field AE. Physical activity in relation to longterm weight maintenance after intentional weight loss in premenopausal women. Obesity (Silver Spring, Md). 2010; 18: 167-74.

92. Lusk AC, Mekary RA, Feskanich D and Willett WC. Bicycle riding, walking, and weight gain in premenopausal women. Arch Intern Med. 2010; 170: 1050-6.

93. The Norwegian Directorate of Health. Recommendations on diet, nutrition and physical activity. 2014.

94. Saris WH, Blair SN, van Baak MA, et al. How much physical activity is enough to prevent unhealthy weight gain? Outcome of the IASO 1st Stock Conference and consensus statement. Obesity reviews : an official journal of the International Association for the Study of Obesity. 2003; 4: 101-14. 95. Wing RR. Behavioral weight control. In: Wadden TA, Stunkard, A.J, , (ed.). Handbook of obesity treatment. New York: Guilford Press, 2002, p. 301-16.

96. Foster GD, Makris AP and Bailer BA. Behavioral treatment of obesity. Am J Clin Nutr. 2005; 82: 230s-5s.

97. Wadden TA and Foster GD. Behavioral treatment of obesity. Med Clin North Am. 2000; 84: 441-61, vii.

98. Rubak S, Sandbaek A, Lauritzen T and Christensen B. Motivational interviewing: a systematic review and meta-analysis. Br J Gen Pract. 2005; 55: 305-12.

99. Svetkey LP, Stevens VJ, Brantley PJ, et al. Comparison of strategies for sustaining weight loss: the weight loss maintenance randomized controlled trial. JAMA. 2008; 299: 1139-48.

100. Wing RR and Phelan S. Long-term weight loss maintenance. Am J Clin Nutr. 2005; 82: 222S-

$5 \mathrm{~S}$.

101. Knowler WC, Barrett-Connor E, Fowler SE, et al. Reduction in the incidence of type 2 diabetes with lifestyle intervention or metformin. N Engl J Med. 2002; 346: 393-403.

102. Lindstrom J, llanne-Parikka P, Peltonen M, et al. Sustained reduction in the incidence of type 2 diabetes by lifestyle intervention: follow-up of the Finnish Diabetes Prevention Study. Lancet. 2006; 368: 1673-9.

103. Wing RR. Long-term effects of a lifestyle intervention on weight and cardiovascular risk factors in individuals with type 2 diabetes mellitus: four-year results of the Look AHEAD trial. Arch Intern Med. 2010; 170: 1566-75.

104. Wing RR, Bolin P, Brancati FL, et al. Cardiovascular effects of intensive lifestyle intervention in type 2 diabetes. N Engl J Med. 2013; 369: 145-54.

105. Christiansen T, Bruun JM, Madsen EL and Richelsen B. Weight loss maintenance in severely obese adults after an intensive lifestyle intervention: 2- to 4-year follow-up. Obesity (Silver Spring, Md). 2007; 15: 413-20.

106. Goodpaster BH, Delany JP, Otto AD, et al. Effects of diet and physical activity interventions on weight loss and cardiometabolic risk factors in severely obese adults: a randomized trial. JAMA. 2010; 304: 1795-802.

107. Martins C, Strommen M, Stavne OA, Nossum R, Marvik R and Kulseng B. Bariatric surgery versus lifestyle interventions for morbid obesity--changes in body weight, risk factors and comorbidities at 1 year. Obes Surg. 2011; 21: 841-9.

108. Ryan DH, Johnson WD, Myers VH, et al. Nonsurgical weight loss for extreme obesity in primary care settings: results of the Louisiana Obese Subjects Study. Arch Intern Med. 2010; 170: 146-54.

109. Hofso D, Nordstrand N, Johnson LK, et al. Obesity-related cardiovascular risk factors after weight loss: a clinical trial comparing gastric bypass surgery and intensive lifestyle intervention. Eur J Endocrinol. 2010; 163: 735-45. 
110. Hakala P. Weight reduction programmes at a rehabilitation centre and a health centre based on group counselling and individual support: short- and long-term follow-up study. Int J Obes Relat Metab Disord. 1994; 18: 483-9.

111. Pi-Sunyer X, Blackburn G, Brancati FL, et al. Reduction in weight and cardiovascular disease risk factors in individuals with type 2 diabetes: one-year results of the look AHEAD trial. Diabetes Care. 2007; 30: 1374-83.

112. Danielsen KK, Svendsen M, Maehlum S and Sundgot-Borgen J. Changes in body composition, cardiovascular disease risk factors, and eating behavior after an intensive lifestyle intervention with high volume of physical activity in severely obese subjects: a prospective clinical controlled trial. Journal of obesity. 2013; 2013: 325464.

113. Buchwald $\mathrm{H}$ and Buchwald JN. Evolution of operative procedures for the management of morbid obesity 1950-2000. Obes Surg. 2002; 12: 705-17.

114. Jenkins NL, Johnson JO, Mageau RP, Bowen JB and Pofahl WE. Who's who in bariatric surgery: the pioneers in the development of surgery for weight control. Curr Surg. 2005; 62: 38-44.

115. Scopinaro N. The IFSO and obesity surgery throughout the world. International Federation for the Surgery of Obesity. Obes Surg. 1998; 8: 3-8.

116. Akkary E. Bariatric surgery evolution from the malabsorptive to the hormonal era. Obes Surg. 2012; 22: 827-31.

117. Mason EE and Ito C. Gastric bypass. Ann Surg. 1969; 170: 329-39.

118. Sjostrom L, Narbro K, Sjostrom CD, et al. Effects of bariatric surgery on mortality in Swedish obese subjects. N Engl J Med. 2007; 357: 741-52.

119. Aasheim ET, Mala T, Sovik TT, Kristinsson J and Bohmer T. [Surgical treatment of morbid obesity]. Tidsskr Nor Laegeforen. 2007; 127: 38-42.

120. Giske L LV, Elvsaas IK $\varnothing$, Hofmann B, Håvelsrud K, Vang V, Fure B. Long-term effects of bariatric surgery. 2014.

121. The National Council for Priority Setting in Health Care. 2014.

122. Singal AG, Higgins PD and Waljee AK. A primer on effectiveness and efficacy trials. Clinical and translational gastroenterology. 2014; 5: e45.

123. Zwarenstein $\mathrm{M}$, Treweek $\mathrm{S}$, Gagnier JJ, et al. Improving the reporting of pragmatic trials: an extension of the CONSORT statement. BMJ. 2008; 337: a2390.

124. Gjevestad E, Karlsen TI, Røislien J, Mæhlum S and Hjelmesæth J. The effectiveness of secondary and tertiary care lifestyle intervention in morbidly obese patients: a 1-year nonrandomized controlled pragmatic clinical trial. Clinical Obesity. 2013; 3: 39-50.

125. Gjevestad E, Hjelmesæth J, Sandbu R and Nordstrand N. Effects of intensive lifestyle intervention and gastric bypass on aortic stiffness: A 1-year nonrandomized clinical study. Obesity. 2014: n/a-n/a.

126. Schofield WN. Predicting basal metabolic rate, new standards and review of previous work. Hum Nutr Clin Nutr. 1985; 39 Suppl 1: 5-41.

127. Petersen L, Schnohr P and Sorensen TI. Longitudinal study of the long-term relation between physical activity and obesity in adults. Int J Obes Relat Metab Disord. 2004; 28: 105-12.

128. The Norwegian Directorate of Health. Kostråd for å fremme folkehelsen of forebygge kroniske sykdommer i Norge. Helsedirektoratet. 2011.

129. Hassett AL and Gevirtz RN. Nonpharmacologic treatment for fibromyalgia: patient education, cognitive-behavioral therapy, relaxation techniques, and complementary and alternative medicine.

Rheum Dis Clin North Am. 2009; 35: 393-407.

130. IFAPA. The International Federation of Adapted Physical Activity.

131. The Morbid Obesity Center, Vestfold Hospital Trust.

132. Matthews DR, Hosker JP, Rudenski AS, Naylor BA, Treacher DF and Turner RC. Homeostasis model assessment: insulin resistance and beta-cell function from fasting plasma glucose and insulin concentrations in man. Diab tologia. 1985; 28: 412-9. 
133. Friedewald WT, Levy RI and Fredrickson DS. Estimation of the concentration of low-density lipoprotein cholesterol in plasma, without use of the preparative ultracentrifuge. Clin Chem. 1972; 18: 499-502.

134. Ware J, Jr., Kosinski M and Keller SD. A 12-Item Short-Form Health Survey: construction of scales and preliminary tests of reliability and validity. Med Care. 1996; 34: 220-33.

135. Niero $M$, Martin $M$, Finger $T$, et al. A new approach to multicultural item generation in the development of two obesity-specific measures: the Obesity and Weight Loss Quality of Life (OWLQOL) questionnaire and the Weight-Related Symptom Measure (WRSM). Clin Ther. 2002; 24: 690-700.

136. Patrick DL, Bushnell DM and Rothman M. Performance of two self-report measures for evaluating obesity and weight loss. Obes Res. 2004; 12: 48-57.

137. Duval K, Marceau P, Perusse L and Lacasse Y. An overview of obesity-specific quality of life questionnaires. Obesity reviews : an official journal of the International Association for the Study of Obesity. 2006; 7: 347-60.

138. Stucki A, Borchers M, Stucki G, Cieza A, Amann E and Ruof J. Content comparison of health status measures for obesity based on the international classification of functioning, disability and health. Int J Obes (Lond). 2006; 30: 1791-9.

139. Solberg M AS. Development of methods for measuring physical activity. Development and validation of a questionnaire for adolescents and adults. 2002.

140. Naess O, Sogaard AJ, Arnesen E, et al. Cohort profile: cohort of Norway (CONOR). Int J Epidemiol. 2008; 37: 481-5.

141. Najjar SS, Scuteri A and Lakatta EG. Arterial aging: is it an immutable cardiovascular risk factor? Hypertension. 2005; 46: 454-62.

142. Sutton-Tyrrell K, Najjar SS, Boudreau RM, et al. Elevated aortic pulse wave velocity, a marker of arterial stiffness, predicts cardiovascular events in well-functioning older adults. Circulation. 2005; 111: 3384-90.

143. Meaume S, Benetos A, Henry OF, Rudnichi A and Safar ME. Aortic pulse wave velocity predicts cardiovascular mortality in subjects $>70$ years of age. Arterioscler Thromb Vasc Biol. 2001; 21: 2046-50.

144. Blacher J, Guerin AP, Pannier B, Marchais SJ, Safar ME and London GM. Impact of aortic stiffness on survival in end-stage renal disease. Circulation. 1999; 99: 2434-9.

145. Laurent S, Boutouyrie $\mathrm{P}, \mathrm{Asmar} \mathrm{R}$, et al. Aortic stiffness is an independent predictor of allcause and cardiovascular mortality in hypertensive patients. Hypertension. 2001; 37: 1236-41.

146. Mansia G, De Backer G, Dominiczak A, et al. 2007 ESH-ESC Guidelines for the management of arterial hypertension: the task force for the management of arterial hypertension of the European Society of Hypertension (ESH) and of the European Society of Cardiology (ESC). Blood Press. 2007; 16: 135-232.

147. Boutouyrie $\mathrm{P}$, Tropeano Al, Asmar R, et al. Aortic stiffness is an independent predictor of primary coronary events in hypertensive patients: a longitudinal study. Hypertension. 2002; 39: 10-5. 148. Willum-Hansen T, Staessen JA, Torp-Pedersen C, et al. Prognostic value of aortic pulse wave velocity as index of arterial stiffness in the general population. Circulation. 2006; 113: 664-70.

149. Laurent S, Katsahian S, Fassot C, et al. Aortic stiffness is an independent predictor of fatal stroke in essential hypertension. Stroke. 2003; 34: 1203-6.

150. Laurent $S$ and Boutouyrie $P$. Arterial stiffness: a new surrogate end point for cardiovascular disease? Journal of nephrology. 2007; 20 Suppl 12: S45-50.

151. Mattace-Raso FU, van der Cammen TJ, Hofman A, et al. Arterial stiffness and risk of coronary heart disease and stroke: the Rotterdam Study. Circulation. 2006; 113: 657-63.

152. Hansen TW, Li Y, Staessen JA, et al. Independent prognostic value of the ambulatory arterial stiffness index and aortic pulse wave velocity in a general population. $J$ Hum Hypertens. 2008; 22: 214-6. 
153. Wang Z, Deurenberg P, Wang W, Pietrobelli A, Baumgartner RN and Heymsfield SB. Hydration of fat-free body mass: review and critique of a classic body-composition constant. Am J Clin Nutr. 1999; 69: 833-41.

154. Barinas-Mitchell E, Kuller LH, Sutton-Tyrrell K, et al. Effect of weight loss and nutritional intervention on arterial stiffness in type 2 diabetes. Diabetes Care. 2006; 29: 2218-22.

155. Vickers AJ and Altman DG. Statistics notes: Analysing controlled trials with baseline and follow up measurements. BMJ. 2001; 323: 1123-4.

156. Ranstam J, Turkiewicz A, Boonen S, Van Meirhaeghe J, Bastian L and Wardlaw D. Alternative analyses for handling incomplete follow-up in the intention-to-treat analysis: the randomized controlled trial of balloon kyphoplasty versus non-surgical care for vertebral compression fracture (FREE). BMC medical research methodology. 2012; 12: 35.

157. Wyrwich KW, Bullinger M, Aaronson N, Hays RD, Patrick DL and Symonds T. Estimating clinically significant differences in quality of life outcomes. Qual Life Res. 2005; 14: 285-95.

158. Kazis LE, Anderson JJ and Meenan RF. Effect sizes for interpreting changes in health status. Med Care. 1989; 27: S178-89.

159. Cohen JC, P. West, SG. Aiken, LS. Applied Multiple Regression/correlation Analysis for the Behavioral Sciences, third edition. London: Lawrence Erlbaum Associates, 2003.

160. World Medical Association declaration of Helsinki. Recommendations guiding physicians in biomedical research involving human subjects. JAMA. 1997; 277: 925-6.

161. Schulz KF, Altman DG and Moher D. CONSORT 2010 statement: updated guidelines for reporting parallel group randomized trials. Ann Intern Med. 2010; 152: 726-32.

162. Porzsolt $F$, Eisemann $M$, Habs $M$ and Wyer P. Form follows function: pragmatic controlled trials (PCTs) have to answer different questions and require different designs than randomized controlled trials (RCTs). Zeitschrift fur Gesundheitswissenschaften = Journal of public health. 2013; 21: 307-13.

163. Black N. Patient reported outcome measures could help transform healthcare. BMJ. 2013; 346: f167.

164. Rothwell PM. External validity of randomised controlled trials: "to whom do the results of this trial apply?". Lancet. 2005; 365: 82-93.

165. McKee M, Britton A, Black N, McPherson K, Sanderson C and Bain C. Methods in health services research. Interpreting the evidence: choosing between randomised and non-randomised studies. BMJ. 1999; 319: 312-5.

166. Ware JH and Hamel MB. Pragmatic trials--guides to better patient care? N Eng/ J Med. 2011; 364: 1685-7.

167. Tunis SR, Stryer DB and Clancy CM. Practical clinical trials: increasing the value of clinical research for decision making in clinical and health policy. JAMA. 2003; 290: 1624-32.

168. Miller RG. Simultaneous Statistical Inference. Springer Verlag New York, 1981.

169. Graham JW. Missing data analysis: making it work in the real world. Annu Rev Psychol. 2009; 60: 549-76.

170. Schafer JL and Graham JW. Missing data: our view of the state of the art. Psychological methods. 2002; 7: 147-77.

171. Pietilainen $\mathrm{KH}$, Korkeila $\mathrm{M}, \mathrm{Bog} \mathrm{LH}$, et al. Inaccuracies in food and physical activity diaries of obese subjects: complementary evidence from doubly labeled water and co-twin assessments. Int J Obes (Lond). 2010; 34: 437-45.

172. Karlsen TI, Tveita EK, Natvig GK, Tonstad S and Hjelmesaeth J. Validity of the SF-36 in patients with morbid obesity. Obes Facts. 2011; 4: 346-51.

173. Wee CC, Davis RB and Hamel MB. Comparing the SF-12 and SF-36 health status questionnaires in patients with and without obesity. Health and quality of life outcomes. 2008; 6: 11.

174. Chiu YC, Arand PW, Shroff SG, Feldman T and Carroll JD. Determination of pulse wave velocities with computerized algorithms. Am Heart J. 1991; 121: 1460-70.

175. Van Bortel LM, Duprez D, Starmans-Kool MJ, et al. Clinical applications of arterial stiffness, Task Force III: recommendations for user procedures. Am J Hypertens. 2002; 15: 445-52. 
176. Heath EM, Adams TD, Daines MM and Hunt SC. Bioelectric impedance and hydrostatic weighing with and without head submersion in persons who are morbidly obese. J Am Diet Assoc. 1998; 98: 869-75.

177. Volgyi E, Tylavsky FA, Lyytikainen A, Suominen H, Alen M and Cheng S. Assessing body composition with DXA and bioimpedance: effects of obesity, physical activity, and age. Obesity (Silver Spring, Md). 2008; 16: 700-5.

178. Piccoli A, Brunani A, Savia G, et al. Discriminating between body fat and fluid changes in the obese adult using bioimpedance vector analysis. Int J Obes Relat Metab Disord. 1998; 22: 97-104. 179. Kurtze N, Rangul V, Hustvedt BE and Flanders WD. Reliability and validity of self-reported physical activity in the Nord-Trondelag Health Study (HUNT 2). Eur J Epidemiol. 2007; 22: 379-87. 180. Graff-Iversen S, Anderssen SA, Holme IM, Jenum AK and Raastad T. Two short questionnaires on leisure-time physical activity compared with serum lipids, anthropometric measurements and aerobic power in a suburban population from Oslo, Norway. Eur J Epidemiol. 2008; 23: 167-74. 181. Aadland E, Jepsen R, Andersen JR and Anderssen SA. Increased physical activity improves aerobic fitness, but not functional walking capacity, in severely obese subjects participating in a lifestyle intervention. Journal of rehabilitation medicine : official journal of the UEMS European Board of Physical and Rehabilitation Medicine. 2013; 45: 1071-7.

182. Tur JJ, Escudero AJ, Alos MM, et al. One year weight loss in the TRAMOMTANA study. A randomized controlled trial. Clin Endocrinol (Oxf). 2013; 79: 791-9.

183. Maffiuletti NA, Agosti F, Marinone PG, Silvestri G, Lafortuna CL and Sartorio A. Changes in body composition, physical performance and cardiovascular risk factors after a 3-week integrated body weight reduction program and after 1-y follow-up in severely obese men and women. Eur J Clin Nutr. 2005; 59: 685-94.

184. Kolotkin RL, Crosby RD and Williams GR. Health-related quality of life varies among obese subgroups. Obes Res. 2002; 10: 748-56.

185. Faulconbridge LF, Wadden TA, Thomas JG, Jones-Corneille LR, Sarwer DB and Fabricatore AN. Changes in depression and quality of life in obese individuals with binge eating disorder: bariatric surgery versus lifestyle modification. Surgery for obesity and related diseases : official journal of the American Society for Bariatric Surgery. 2013; 9: 790-6.

186. Halperin F, Ding SA, Simonson DC, et al. Roux-en-Y Gastric Bypass Surgery or Lifestyle With Intensive Medical Management in Patients With Type 2 Diabetes: Feasibility and 1-Year Results of a Randomized Clinical Trial. JAMA surgery. 2014; 149: 716-26.

187. Dengo AL, Dennis EA, Orr JS, et al. Arterial destiffening with weight loss in overweight and obese middle-aged and older adults. Hypertension. 2010; 55: 855-61.

188. McNamee R. Confounding and confounders. Occup Environ Med. 2003; 60: 227-34; quiz 164,

234.

189. Yoshizawa M, Maeda S, Miyaki A, et al. Effect of 12 weeks of moderate-intensity resistance training on arterial stiffness: a randomised controlled trial in women aged 32-59 years. Br J Sports Med. 2009; 43: 615-8.

190. Guimaraes GV, Ciolac EG, Carvalho VO, D'Avila VM, Bortolotto LA and Bocchi EA. Effects of continuous vs. interval exercise training on blood pressure and arterial stiffness in treated hypertension. Hypertens Res. 2010; 33: 627-32.

191. Hughes TM, Althouse AD, Niemczyk NA, Hawkins MS, Kuipers AL and Sutton-Tyrrell K. Effects of weight loss and insulin reduction on arterial stiffness in the SAVE trial. Cardiovascular diabetology. 2012; 11: 114.

192. Cooper JN, Buchanich JM, Youk A, et al. Reductions in arterial stiffness with weight loss in overweight and obese young adults: potential mechanisms. Atherosclerosis. 2012; 223: 485-90.

193. Tanaka $\mathrm{H}$ and Safar ME. Influence of lifestyle modification on arterial stiffness and wave reflections. Am J Hypertens. 2005; 18: 137-44.

194. Lakatta EG. Arterial and cardiac aging: major shareholders in cardiovascular disease enterprises: Part III: cellular and molecular clues to heart and arterial aging. Circulation. 2003; 107: 490-7. 
195. Safar ME, Laurent S, Pannier BM and London GM. Structural and functional modifications of peripheral large arteries in hypertensive patients. J Clin Hypertens. 1987; 3: 360-7.

196. Wycherley TP, Brinkworth GD, Keogh JB, Noakes M, Buckley JD and Clifton PM. Long-term effects of weight loss with a very low carbohydrate and low fat diet on vascular function in overweight and obese patients. J Intern Med. 2010; 267: 452-61.

197. Seidlerova J, Filipovsky J, Mayer O, Wohlfahrt P and Cifkova R. Positive effects of antihypertensive treatment on aortic stiffness in the general population. Hypertens Res. 2014; 37: 648.

198. Agarwal N, Rice SP, Bolusani $\mathrm{H}$, et al. Metformin reduces arterial stiffness and improves endothelial function in young women with polycystic ovary syndrome: a randomized, placebocontrolled, crossover trial. J Clin Endocrinol Metab. 2010; 95: 722-30.

199. Rider OJ, Tayal U, Francis JM, et al. The effect of obesity and weight loss on aortic pulse wave velocity as assessed by magnetic resonance imaging. Obesity (Silver Spring, Md). 2010; 18: 2311-6.

200. Wang R, Lagakos SW, Ware JH, Hunter DJ and Drazen JM. Statistics in medicine--reporting of subgroup analyses in clinical trials. N Engl J Med. 2007; 357: 2189-94.

201. Emdin M, Gastaldelli A, Muscelli E, et al. Hyperinsulinemia and autonomic nervous system dysfunction in obesity: effects of weight loss. Circulation. 2001; 103: 513-9.

202. Hirsch J, Leibel RL, Mackintosh R and Aguirre A. Heart rate variability as a measure of autonomic function during weight change in humans. Am J Physiol. 1991; 261: R1418-23.

203. Arone LJ, Mackintosh R, Rosenbaum M, Leibel RL and Hirsch J. Autonomic nervous system activity in weight gain and weight loss. Am J Physiol. 1995; 269: R222-5.

204. Nilsson PM, Boutouyrie $P$, Cunha $P$, et al. Early vascular ageing in translation: from laboratory investigations to clinical applications in cardiovascular prevention. J Hypertens. 2013; 31: 1517-26.

205. Nilsson PM, Boutouyrie P and Laurent S. Vascular aging: A tale of EVA and ADAM in cardiovascular risk assessment and prevention. Hypertension. 2009; 54: 3-10.

206. Nilsson PM, Lurbe E and Laurent S. The early life origins of vascular ageing and cardiovascular risk: the EVA syndrome. J Hypertens. 2008; 26: 1049-57.

207. Mangoni AA, Mircoli L, Giannattasio C, Ferrari AU and Mancia G. Heart rate-dependence of arterial distensibility in vivo. J Hypertens. 1996; 14: 897-901.

208. Nichols WW, Nicolini FA and Pepine CJ. Determinants of isolated systolic hypertension in the elderly. J Hypertens Suppl. 1992; 10: S73-7.

209. Franklin SS, Khan SA, Wong ND, Larson MG and Levy D. Is pulse pressure useful in predicting risk for coronary heart Disease? The Framingham heart study. Circulation. 1999; 100: 354-60.

210. Abbatecola AM, Chiodini P, Gallo C, et al. Pulse wave velocity is associated with muscle mass decline: Health ABC study. Age (Dordrecht, Netherlands). 2012; 34: 469-78.

211. Ochi M, Kohara K, Tabara $\mathrm{Y}$, et al. Arterial stiffness is associated with low thigh muscle mass in middle-aged to elderly men. Atherosclerosis. 2010; 212: 327-32.

212. Volpi E, Nazemi R and Fujita S. Muscle tissue changes with aging. Current opinion in clinical nutrition and metabolic care. 2004; 7: 405-10.

213. Stehouwer CD, Henry RM and Ferreira I. Arterial stiffness in diabetes and the metabolic syndrome: a pathway to cardiovascular disease. Diab tologia. 2008; 51: 527-39.

214. Aronson D. Cross-linking of glycated collagen in the pathogenesis of arterial and myocardial stiffening of aging and diabetes. J Hypertens. 2003; 21: 3-12.

215. Zieman SJ, Melenovsky V and Kass DA. Mechanisms, pathophysiology, and therapy of arterial stiffness. Arterioscler Thromb Vasc Biol. 2005; 25: 932-43.

216. Tanaka H, Dinenno FA, Monahan KD, Clevenger CM, DeSouza CA and Seals DR. Aging, habitual exercise, and dynamic arterial compliance. Circulation. 2000; 102: 1270-5.

217. Gates PE and Seals DR. Decline in large elastic artery compliance with age: a therapeutic target for habitual exercise. Br J Sports Med. 2006; 40: 897-9.

218. Green DJ, O'Driscoll G, Joyner MJ and Cable NT. Exercise and cardiovascular risk reduction: time to update the rationale for exercise? Journal of applied physiology (Bethesda, Md : 1985). 2008; 105: 766-8. 
219. Meaney E, Vela A, Samaniego V, et al. Metformin, arterial function, intima-media thickness and nitroxidation in metabolic syndrome: the mefisto study. Clin Exp Pharmacol Physiol. 2008; 35: 895-903.

220. Kanaki Al, Sarafidis PA, Georgianos PI, et al. Effects of low-dose atorvastatin on arterial stiffness and central aortic pressure augmentation in patients with hypertension and hypercholesterolemia. Am J Hypertens. 2013; 26: 608-16. 

I 



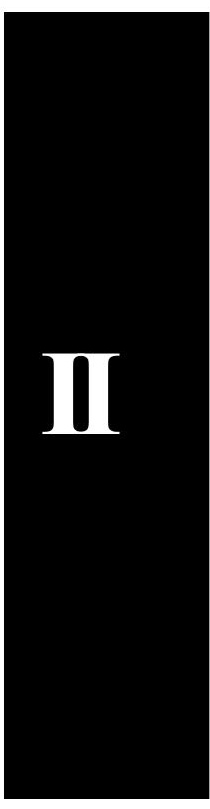





\title{
Arterial Stiffness, Lifestyle Intervention and a Low-Calorie Diet in Morbidly Obese Patients-A Nonrandomized Clinical Trial
}

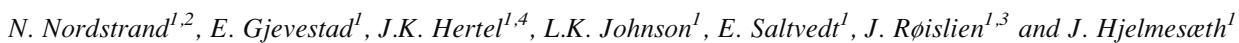

Objective: Arterial stiffness is an independent predictor of cardiovascular morbidity and mortality. This study aimed to compare the 7-week effect of a low-calorie diet (LCD) and an intensive lifestyle intervention program (ILI) on arterial stiffness in morbidly obese individuals.

Design and Methods: Nonrandomized clinical trial. The LCD provided $900 \mathrm{kcal} / \mathrm{day}$, and participants in the LCD group were instructed to maintain their habitual physical activity level. The ILI included two 90min supervised training sessions 3 days a week at moderate to high intensity (4-8 METs) and a caloric restriction of $1000 \mathrm{kcal} /$ day.

Results: A total of 179 individuals completed the study, 88 (56 women) in the ILI group and 91 (57 women) in the LCD group. High-fidelity applanation tonometry (Millar ${ }^{\circledR}$, Sphygmocor ${ }^{\circledR}$ ) was $_{\text {used to }}$ measure carotid-femoral pulse wave velocity (PWV). After adjustment for relevant confounders, the ILI group had a significantly greater reduction in PWV than the LCD group; $-0.4(-0.6,-0.1) \mathrm{m} / \mathrm{s}, P=$ 0.004. When compared to the LCD group, the ILI group showed a larger reduction in systolic and diastolic blood pressure $-5(-9,-1)$ and $-5(-7,-2) \mathrm{mmHg}, P=0.038$ and $P \leq 0.001$ respectively, whereas no difference was observed regarding pulse pressure, $P=0.661$. No significant differences between groups were found regarding the loss of fat mass, $P=0.259$, but the loss of muscle mass was larger in the LCD group, $0.8(0.5,1.1) \mathrm{kg}, P \leq 0.001$.

Conclusion: Despite the limitations of a nonrandomized design, our findings indicate that for morbidly obese individuals a moderate caloric restriction combined with aerobic physical exercise is associated with a greater decline in PWV than a LCD alone.

Obesity (2013) 21, 690-697. doi:10.1002/oby.20099

\section{Background}

Obesity is a world-wide health concern (1) and imparts a degree of cardiovascular risk similar to that associated with hypertension, hyperlipidemia, and smoking (2). New insights from the National Health and Nutrition Examination Survey indicate that the prevalence of obesity has stabilized during the last decade, but there is evidence that within the obese population, the prevalence of morbid obesity is increasing at a high rate $(3,4)$.

The cornerstone of obesity management is weight loss. Any intervention with the intention to cause weight loss must tip the balance between energy intake and expenditure to be successful. Official recommendations state that a reduction in energy intake of $\sim 500 \mathrm{kcal}$ below energy expenditure per day will result in a weight loss of $0.5 \mathrm{~kg}$ per week. There is, however, evidence that the simplicity of the shift in energy balance could be misleading and might not be adaptable to a real-life situation. The weight response to caloric restriction is slow, and to reach steady-state weight, an individual must comply with the dietary intervention for a long time. Purely dietary interventions will be more time consuming and demanding in morbidly obese individuals than in less obese individuals (5). It is therefore important to investigate whether or not there are supplementary efforts (other than a strict diet) that can attenuate the cardiovascular risk associated with morbid obesity. Several lines of evidence indicate that aerobic physical activity might decrease cardiovascular risk in obese individuals $(6,7)$. Whether or not this is true for morbidly obese individuals is unknown.

Arterial stiffness assessed by aortic pulse wave velocity (PWV) is an independent predictor of cardiovascular morbidity and mortality (8), and PWV is increasingly recognized as a valid surrogate endpoint of cardiovascular disease $(9,10)$. In healthy young adults, arteries stiffen when weight is gained and soften with weight loss.

\footnotetext{
${ }^{1}$ Morbid Obesity Center, Vestfold Hospital Trust, Tønsberg, Norway. Correspondence: N. Nordstrand (njord.nordstrand@siv.no and njonor@online.no)

${ }^{2}$ Department of Medicine, Vestfold Hospital Trust, Tønsberg, Norway ${ }^{3}$ Department of Biostatistics, Institute of Basic Medical Sciences, University of Oslo,

Norway ${ }^{4}$ Department of Heart Disease, Haukeland University Hospital, Bergen, Norway
} 
(11) Recent evidence suggests this to hold true in overweight and obese middle-aged individuals $(12,13)$. It has been shown that when matched for age, PWV is $\sim 0.5 \mathrm{~m} / \mathrm{s}$ higher in obese compared with non-obese. This increase in PWV is equivalent to 5-10 years of ageing (14). It has also been shown that aerobic physical activity is associated with the prevention of arterial stiffening (15), and moreover that aerobic training can reduce arterial stiffness (16).

The measurement of PWV is generally accepted as a simple, noninvasive, robust, and reproducible method to determine arterial stiffness and is considered to be the golden standard method for evaluation of arterial stiffness $(17,18)$. To the best of our knowledge, the current study is the first to investigate how a low-calorie diet (LCD) and intensive lifestyle intervention (ILI) affect arterial stiffness in a morbidly obese population.

The primary objective of this study was to compare the effect of a LCD and an ILI program on arterial stiffness in morbidly obese individuals. Our main hypothesis was that a 7-week ILI program consisting of aerobic endurance training 3 days a week and a moderate daily caloric restriction would have a greater effect on arterial stiffness than a 7-week LCD.

We further hypothesized that the loss of skeletal muscle mass and fat mass would be greater in the group subjected to a LCD.

\section{Methods}

\section{Study design}

This is the first part of a nonrandomized clinical trial comparing the effects of an intensive lifestyle modification program, a LCD, and bariatric surgery on arterial stiffness in morbidly obese patients. Results from the baseline cross-sectional analysis have been published elsewhere (19). (ClinicalTrials.gov Identifier NCT00626964).

\section{Setting}

The study was performed at a tertiary care centre (the Morbid Obesity Centre, Vestfold Hospital Trust, Tønsberg, Norway) between February 2008 and February 2011. Before inclusion patients were either assigned to a comprehensive lifestyle modification program at the Clinic of Physical Medicine and Rehabilitation, Vestfold Hospital Trust, or to a LCD followed by bariatric surgery at the Morbid Obesity Centre.

\section{Participants}

All participants were recruited from our tertiary care centre and had to reside within $100 \mathrm{~km}$ of either the hospital for rehabilitation or our clinic. The patients in the ILI group were all selected from subjects that had registered for participation in a standardized health promotion and weight reduction program at the hospital for rehabilitation. Participants in the LCD group were selected from patients due to undergo bariatric surgery at our hospital. The decision regarding the type of intervention was made prior to inclusion to our study and was not a part of our protocol.

Inclusion criteria were body mass index (BMI) $\geq 40.0 \mathrm{~kg} / \mathrm{m}^{2}$ or BMI $\geq 35.0 \mathrm{~kg} / \mathrm{m}^{2}$ accompanied by one or more obesity-related comorbidities.

Exclusion criteria were uncompensated heart failure, cardiac arrhythmias, unstable angina, end-stage renal disease, known bleeding dis- turbances, serious psychiatric disorders, serious eating disorders, cardiac pacemakers, intra-cardiac devices, cerebrovascular event, or a myocardial infarction within the last 6 months.

The study was approved by the regional ethics committee of the Southern Norway Regional Health Authority (code: S-05175) and was performed in accordance with the Declaration of Helsinki (20). Written informed consent was provided by all participants.

\section{Intensive lifestyle intervention}

Each of the ILI groups comprised of 12-14 patients. The baseline individual energy expenditure was calculated according to Schofield's equation (21). Schofield's equation uses information pertaining to the patient's gender, age, and body weight to calculate resting metabolic rate (BMR). To calculate total energy expenditure, BMR was multiplied with a physical activity factor of 1.4 , which is considered the increased energy cost of sedentary behavior (22). All participants received a dietary plan with an energy restriction of $1000 \mathrm{kcal} /$ day in relation to calculated baseline energy expenditure, otherwise according to Norwegian nutritional guidelines (23).

Individuals in the ILI group were followed for 3 days per week during an intervention period of 7 weeks. Each day of intervention lasted $6 \mathrm{~h}$ and included two 90-min supervised training sessions, educational sessions regarding nutrition and physical activity. The first of the two supervised exercise sessions consisted of weight-bearing activities, such as walking, Nordic-walking, running, ballgames, resistance training, and other various exercises. The second exercise session consisted of water aerobics, swimming, and other water-based physical activities. The main aspect of the physical training was aerobic endurance training with moderate (four metabolic equivalents of task (METs)) to high intensity (eight METs). The resistance training was performed with 15-20 repetitions per exercise (24).

Study participants also had individual sessions with qualified personnel who used a client-centered counseling style known as motivational guidance techniques (25) to invoke behavioral change within the participants.

\section{Low calorie diet intervention}

The LCD consisted of crisp bread combined with low-fat products for breakfast, lunch, and supper. Dinner consisted of fish, poultry, or lean meat combined with potatoes, rice, or pasta and vegetables. These ingredients did not provide $>900 \mathrm{kcal}$ per day, of which $37 \%$ of the energy came from protein, $43 \%$ from carbohydrates, and $20 \%$ from fat. Patients were allowed to increase the size of their meal using vegetables and water. Daily intake of a multivitamin/mineral supplement was recommended. Participants in the LCD group were instructed to maintain their habitual physical activity level throughout the intervention. The study participants were not allowed to attend other dietary or physical interventions prior to or during the 7 weeks of intervention in the present study.

\section{Outcomes}

The main outcome variable was the 7-week change in arterial stiffness measured by PWV. Secondary outcomes were changes in weight, body composition, systolic and diastolic blood pressure, and pulse pressure. 


\section{Data sources and measurements}

All participants underwent a medical examination performed by a physician and a trained nurse. All measurements were performed after an overnight fast. Participants were instructed to withdraw from their prescribed medications and smoking prior to the examination on the day of testing. Blood was collected by venipuncture on the day of medical examination. Weight and height were measured with patients wearing light clothing and no shoes. BMI was calculated as weight in kilograms divided by the square of the height in meters. Waist circumference (WC) was measured midway between the 12th rib and the iliac crest. Blood pressure was measured after 5 min of rest using an electronic auscultatory blood pressure recorder with an appropriately sized cuff based on an arm circumference measurement (Dinamap ${ }^{\circledR}$, ProCare Series, G.E. Medical Systems) with the patient sitting in an upright position. Three measurements were recorded. The average of the second and third recordings was used in the blood pressure analysis. Bioelectrical impedance measures were collected using the Inbody 720 , Body Composition Analyzer, Biospace Co. Ltd. Arterial hypertension was defined by either, a systolic blood pressure $\geq 140 \mathrm{mmHg}$, diastolic blood pressure $\geq 90 \mathrm{mmHg}$, or the use of antihypertensive medication. Ischemic heart disease was defined as a history of stable coronary artery disease, percutaneous coronary intervention, coronary artery bypass graft surgery, or myocardial infarction. Mean arterial pressure (MAP) was calculated as [(diastolic pressure $\times 2)+$ systolic pressure $] / 3$ (11).

The Sphygmocor system (Artcor, Sidney, Australia) and a single high-fidelity applanation tonometer $\left(\right.$ Millar $\left.^{(B)}\right)$ were used to measure PWV. Pulse waves were obtained sequentially from the carotid and femoral artery. The locations of the sternal notch, carotid pulse, and femoral pulse were located. We placed tape directly above these spots with the patient in horizontal position to get a true linear measure. The distance was calculated as the distance between the carotid notch and the femoral artery minus the distance between the carotid notch and the carotid artery (18). The PWV was calculated from the transit time and the distance between these two arterial sites, determined in relation to the R-wave of the ECG, with patients lying in a horizontal position. Blood pressure was measured before every recording and entered into the Sphygmocor recorder to secure valid recordings with similar blood pressure. Three complete sets of data were sampled, and the average value was used as result.

Bioelectrical impedance analysis was obtained using Inbody 720, Body Composition Analyzer, Biospace Co. Ltd. Each patient was undressed except for underwear and placed in an upright position on the body composition analyzer. All jewelry and wrist-watches were removed before recording. The InBody 720 uses the segmental BIA method to examine the body as five cylinders (four limbs and a trunk) and measures impedance in these parts separately. It also uses electrical current at multi-frequency $(5,50,250,500$, and $1000 \mathrm{kHz})$ to estimate the amount of extracellular and intracellular water. Homeostasis Model Assessment Insulin Resistance (HOMA- IR) was calculated as [fasting serum glucose $(\mathrm{mmol} / \mathrm{L}) \times$ fasting serum insulin $(\mathrm{pmol} / \mathrm{L})] / 135(26)$. Low-density lipoprotein cholesterol (LDL) concentrations were estimated by the Friedewald equation: LDL cholesterol = Total cholesterol - HDL cholesterol $-(0.45 \times$ triglycerides $)(27)$. LDL cholesterol was not calculated if $S$-triglycerides were $<0.2 \mathrm{mmol} / \mathrm{L}$ or $\geq 5 \mathrm{mmol} / \mathrm{L}$.

\section{Laboratory analyses}

Analyses of serum glucose and blood lipids were performed using dry reagent slide technology on the Vitros FS 5.1 (Ortho-Clinical
Diagnostics, New York). Glycated hemoglobin (HbAlc) was analyzed using high-performance liquid chromatography on Tosoh HLC-723 G7 (Tosoh Corporation, Tokyo, Japan). Sera for analysis of insulin were stored at $-20^{\circ} \mathrm{C}$ and analyzed within 1 week of blood sampling (Linco Research Inc., St. Charles, MO).

\section{Sample size}

The present study is the first part of an ongoing non-randomized clinical trial. The sample size was calculated based on BarinasMitchell et al.'s (28) study, which showed that a mean weight reduction of $8 \%$ resulted in $1.4 \mathrm{~m} / \mathrm{s}$ mean reduction in PWV in overweight individuals with diabetes. From this, we hypothesized that the mean PWV would be $8.2 \mathrm{~m} / \mathrm{s}$ in both groups at baseline and that a 1-year weight loss of $8 \%$ in the ILI group would lead to a mean (SD) of $1.4(2.8) \mathrm{m} / \mathrm{s}$ reduction in PWV. The expected weight loss following gastric bypass surgery is roughly three times as high, and we assumed that the mean reduction in PWV would be $2.8 \mathrm{~m} / \mathrm{s}$. Accordingly, at least 120 individuals had to complete the study to show a statistically significant difference between the two groups at a power of $80 \%(\alpha=0.05)$. To allow for a drop-out rate of $<40 \%$, we decided to include 200 patients (100 in each group).

\section{Statistical methods}

Data are presented as mean (SD) or number (\%) unless otherwise specified. Skewed data were transformed using natural logarithms to approximate normality. Differences between groups at baseline were analyzed using independent samples $t$-test or Fisher's exact test.

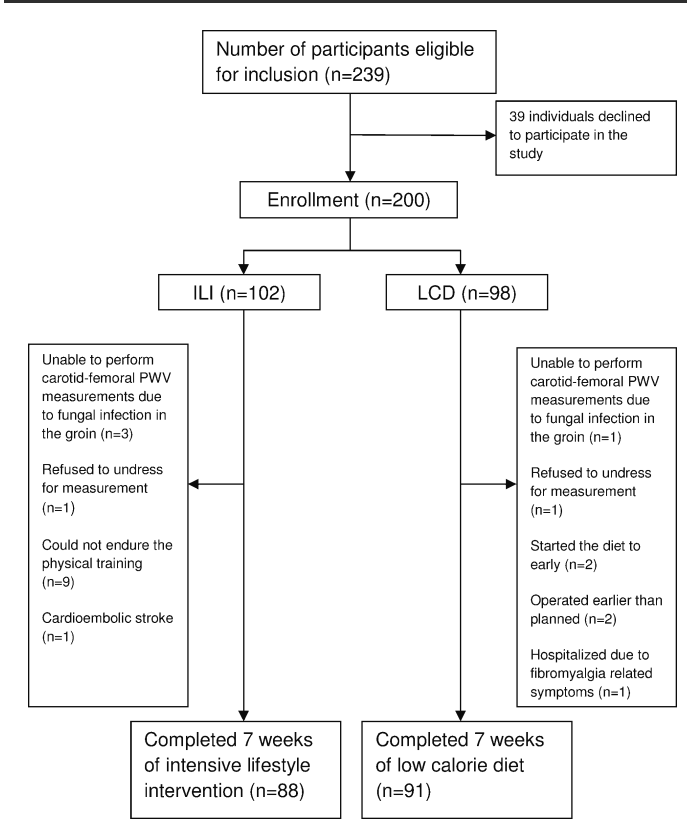

FIGURE 1 Flow chart. 
Spearman's rank correlation was used to assess the bivariate association between weight loss, the change in fat mass, and the change in PWV. Within-group changes from baseline to follow-up were analyzed using paired samples $t$-test. Differences between groups in PWV at follow-up were analyzed using analysis of covariance (ANCOVA) including age, gender, MAP, baseline BMI, history of coronary artery disease, and baseline value of the dependent variable as covariates (11). Directed acyclic graphs (29) and baseline differences between groups were used as regression modeling tools to identify the confounding factors to be included as covariates in the ANCOVA. The model was further adjusted for change in MAP and reduction in weight to explore if the specific intervention was independently associated with the measured effect variable beyond the effect mediated by weight loss or reduction in MAP. Differences between groups in systolic blood pressure, diastolic blood pressure, and pulse pressure were analyzed using ANCOVA including age, gender, history of coronary artery disease, baseline BMI, and baseline value of dependent variable as covariates.

\section{Results}

During the inclusion period, 239 individuals were eligible for inclusion, 39 declined to participate leaving 200 individuals who accepted our invitation to participate in the study (Figure 1). Nine individuals in the ILI group found the activity level too demanding and withdrew from the study. Twelve individuals did not complete the study due to other reasons, leaving a total of 179 patients in the present analysis, 88 (56 women) in the ILI group and 91 (57 women) in the LCD group (Figure 1).

The groups were not significantly different in terms of PWV, but individuals in the LCD group were slightly younger and had higher systolic blood pressure, pulse pressure, slightly higher body weight and fat mass, and lower prevalence of ischemic heart disease compared with the ILI group (Table 1). Our records show that $40(46 \%)$ of the individuals in the ILI group and $31(34 \%)$ in the LCD group used antihypertensive medication at baseline, $P=0.129$. The majority (39 out of 40 and 30 out of 31) were still taking antihypertensive medication at follow up.

\section{Changes in arterial stiffness and blood pressure}

The ILI group had a statistically significant reduction in mean (95\% CI) PWV of $-0.6(-0.8,-0.4) \mathrm{m} / \mathrm{s}, P \leq 0.001$ (Figure 2a). In contrast, the reduction in PWV observed in the LCD group was not statistically significant, $-0.2(-0.4,0.0) \mathrm{m} / \mathrm{s}, P=0.064$. After adjustment for age, gender, baseline MAP, baseline BMI, history of coronary artery disease, and baseline PWV, the decline in PWV was significantly larger in the ILI group than in the LCD group, between-group difference $0.4(0.1,0.6) \mathrm{m} / \mathrm{s}, P=0.004$ (Figure $2 \mathrm{~b}$ ). The larger decline in PWV observed in the ILI group (compared to the LCD group) was slightly attenuated but still significant after further adjustment for change in weight and change in MAP $0.3(0.1,0.6) \mathrm{m} / \mathrm{s}, P=0.019$.

Both interventions led to a statistically significant reduction in systolic blood pressure, but only the ILI group showed a significant reduction in diastolic pressure (Figure $3 \mathrm{a}$ and $\mathrm{b}$ ). When compared to the LCD group, the ILI group showed a larger decline in systolic and diastolic blood pressure after adjustments for age, gender, baseline BMI, history of coronary artery disease, baseline value of the dependent vari-
TABLE 1 Baseline demography and differences between groups in anthropometric measures, carotid-femoral PWV, and blood pressure, medication, and glucose and lipid metabolism

\begin{tabular}{lccc}
\hline & ILI & LCD & $\boldsymbol{P}$ \\
\hline Age & $45.2(10.9)$ & $42.3(9.6)$ & 0.045 \\
Male/female & $32 / 56$ & $34 / 57$ & 0.999 \\
Diabetes & $19(21)$ & $25(27)$ & 0.388 \\
Hypertension & $68(77)$ & $59(65)$ & 0.072 \\
Ischemic heart disease & $12(14)$ & $2(2)$ & 0.005 \\
Smokers & $14(16)$ & $18(20)$ & 0.560
\end{tabular}

Weight and anthropometric measures

Weight, kg 125.2(20.4) $137.8(22.4) \quad \leq 0.001$

Body mass index, $\mathrm{kg} / \mathrm{m}^{2} \quad 42.5(5.1) \quad 45.7(5.5) \quad \leq 0.001$

Waist circumference, $\mathrm{cm} \quad 128(13) \quad 133(13) \quad 0.007$

Fat mass, $\mathrm{kg}$

$58.3(12.4) \quad 66.8(12.9) \quad \leq 0.001$

Skeletal muscle mass, $\mathrm{kg} \quad 37.7(7.3) \quad 39.8(8.0) \quad 0.078$

PWV and blood pressure

$\begin{array}{llll}\text { Carotid-femoral pulse wave } & 8.6(1.8) & 8.7(1.7) & 0.837\end{array}$

velocity, $\mathrm{m} / \mathrm{s}$

Systolic blood pressure, $\mathrm{mm} \mathrm{Hg} \quad 146(17) \quad 139(19) \quad 0.007$

Diastolic blood pressure, $\mathrm{mm} \mathrm{Hg} \quad 80(11) \quad 81$ (15) $\quad 0.613$

Pulse pressure, $\mathrm{mm} \mathrm{Hg} \quad 66(16) \quad 58(16) \quad 0.002$

Medication

$\beta$-Blocker

Calcium-channel blocker

$14(16) \quad 11(12)$

0.521

$10(11) \quad 11(12) \quad 0.999$

Inhibitors of the RAA system $\quad 34(39) \quad 26(29) \quad 0.159$

Diuretics $\quad 18(21) \quad 12(13) \quad 0.232$

$\begin{array}{lll}\text { Statins } & 19(22) & 12(13)\end{array}$

Glucose and lipid metabolism

Insulin, $\mathrm{pmol} / \mathrm{L}$

Glucose, $\mathrm{mmol} / \mathrm{L}$

$94.02(53.2) \quad 117.6(100.1) \quad 0.060$

$\mathrm{HbA1C}, \%$

HOMA-IR

$6.0(2.2) \quad 6.1(2.1) \quad 0.516$

$6.0(1.2) \quad 6.1(1.2) \quad 0.789$

$4.09(2.76) \quad 5.65(5.97) \quad 0.057$

Total cholesterol, mmol/L $\quad 5.1(1.0) \quad 4.9(0.9) \quad 0.156$

LDL cholesterol, mmol/L $\quad 3.1(1.0) \quad 3.1(0.8) \quad 0.611$

HDL cholesterol, mmol/L $\quad 1.2(0.3) \quad 1.1(0.3) \quad 0.074$

Triglycerides, $\mathrm{mmol} / \mathrm{L} \quad 1.8(1.2) \quad 1.6(0.9) \quad 0.703$

Number (\%) or mean (SD). Independent samples t-test or Fischer's exact test.

able, and between-group difference: $5(1,9)$ and $5(2,7) \mathrm{mmHg} ; P=$ 0.038 and $P \leq 0.001$, respectively. The same adjustments were made in terms of decline in pulse pressure and showed no significant difference between groups, $P=0.661$ Table 2 .

\section{Weight, body composition, glucose metabolism, and lipids}

Body weight and WC were significantly reduced after both interventions (Table 3). After adjustments for age, gender, and baseline weight, a significantly larger weight loss was shown in the LCD group, between-group difference of $-1.5(-2.6,-0.5) \mathrm{kg}, P=0.006$. In contrast, the adjusted reduction of WC was not significantly 
(a)

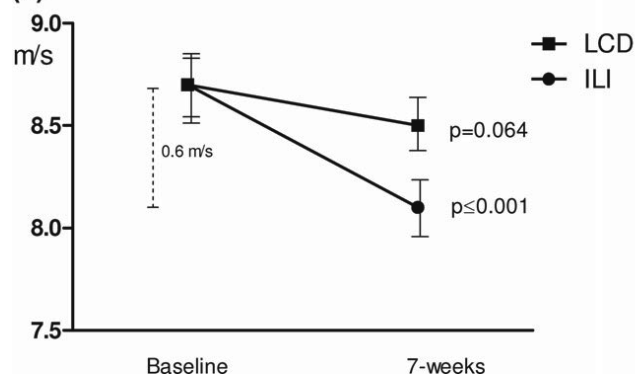

(b)

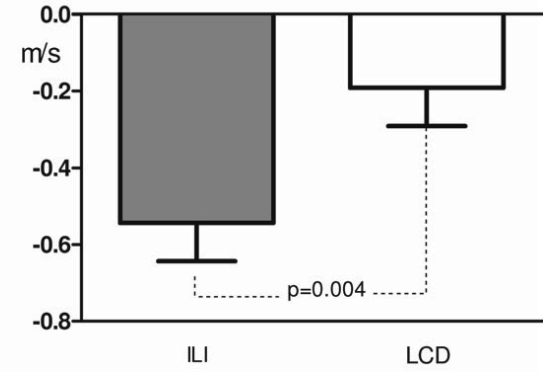

FIGURE 2 Changes in carotid-femoral PWV after 7 weeks of follow up. Within groups (a), adjusted difference between groups (b).

different between groups, $P=0.471$. Bioelectrical impedance analysis showed a significant reduction in fat mass $[-6.0(-6.6,-5.3)$ in the ILI group and $-7.0(-7.8,-6.3)$ in the LCD group] and skeletal muscle mass $[-0.5(-0.7,-0.3)$ in the ILI group and $-1.4(-1.7,-1.2)$ in the LCD group], all $P \leq 0.001$. After adjustments for age, gender, and baseline value of dependent variable, the loss of skeletal muscle mass was $0.8(0.5,1.1) \mathrm{kg}$ larger in the LCD group, $P \leq 0.001$, whereas no differences were apparent in terms of fat mass (Figure 4). Simple correlation analysis showed no significant associations between change in PWV and the changes in weight $(r=-0.017, P=0.821)$ and loss of fat mass $(r=0.041, P=0.590)$. There were reduced blood glucose levels for both interventions with no statistically significant differences between groups (Table 3 ). There was a significantly greater decline in total-cholesterol and triglyceride levels and a smaller decline in HDL-cholesterol for patients in the ILI group compared to those in the LCD group.

\section{Discussion}

Our findings demonstrate that in morbidly obese individuals, a comprehensive 7-week ILI program consisting of a moderate caloric restriction (1000 kcal/day) and aerobic physical activity of moderate to high intensity (4-8 METs) had a larger effect on arterial stiffness than a 7-week LCD ( $<900 \mathrm{kcal} /$ day $)$ intervention. The greater weight loss in the LCD group was due mainly to a significantly greater loss of skeletal muscle mass. No significant between group differences were evident in terms of the decline in fat mass or the reduction in WC.

\section{Comparison with the previous studies}

Some prior studies have shown that PWV can be reduced in less obese through short-term ( 6 weeks to 3 month) weight reduction by the separate use of diets $(12,13)$ and increased aerobic physical exercise (16) or a combination of the two (30). To our knowledge, there are no studies exploring how arterial stiffness can be modified in a population of morbidly obese subjects.

The beneficial results of ILI on PWV are in accordance with the findings of a 1-year randomized clinical trial, which evaluated the effect of a behavioral weight loss intervention program combined with either orlistat or placebo in 38 obese individuals [average BMI 34.0 (5.2) $\mathrm{kg} / \mathrm{m}^{2}$ ] with type 2 diabetes (28). The intervention included an energy restriction of $\sim 500 \mathrm{kcal}$ per day and participants

\section{Systolic pressure}

(a)

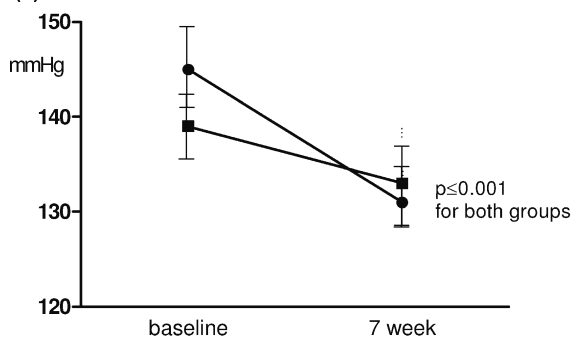

Diastolic pressure

(b)

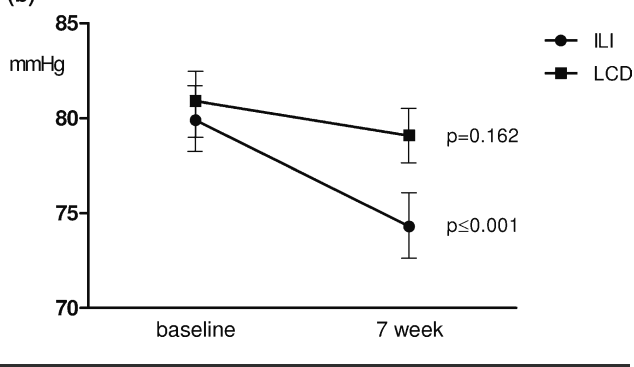

FIGURE 3 The within-group changes in systolic and diastolic blood pressure after 7 weeks of follow-up. 
TABLE 2 Adjusted within- and between-group differences in carotid-femoral pulse wave velocity and blood pressure variables

\begin{tabular}{lcccr}
\hline & ILI & LCD & Adjusted difference & $P$ value \\
\hline PWV, m/s & $-0.6(-0.8,-0.4)$ & $-0.2(-0.4,0.0)$ & $-0.4(-0.6,-0.1)$ & 0.004 \\
Systolic blood pressure, mm Hg & $-13(-16,-11)$ & $-8(-11,-5)$ & $-5(-9,-1)$ & 0.038 \\
Diastolic blood pressure, mm Hg & $-6(-8,-4)$ & $-1(-3,1)$ & $-5(-7,-2)$ & $\leq 0.001$ \\
Pulse pressure, mm Hg & $-6(-9,-3)$ & $-7(-10,-4)$ & $-1(-5,-3)$ & 0.661
\end{tabular}

Mean $(95 \% \mathrm{Cl})$. Analyses of covariance (ANCOVA) including age, gender, baseline BMI, baseline MAP, history of coronary artery disease, and baseline status in dependen variable as covariates for PWV and age, gender, baseline BMl, history of coronary artery disease, and baseline status in dependent variable as covariates for systolic blood pressure, diastolic blood pressure and pulse pressure.

were encouraged to undertake $40-60$ min of moderate intensity physical activity (such as walking or cycling) most week days. Independent of orlistat, the intervention was associated with a mean weight reduction of $7.8 \%(10 \mathrm{~kg})$ and a $0.5 \mathrm{~m} / \mathrm{s}$ reduction in PWV. As in the present study, the changes in PWV did not correlate significantly with weight loss. Importantly, this study differed from our own by the inclusion of relatively few morbidly obese subjects, no supervised training sessions, and a longer follow-up period.

Partly in contrast with our results, a short-term (12 weeks) randomized controlled study of overweight and obese healthy non-smoking middle-aged volunteers showed that a hypocaloric diet intervention (1200-1500 kcal/day) with no emphasis on physical activity was associated with a $7 \mathrm{~kg}$ reduction in weight and a significant reduction in PWV of $1.3 \mathrm{~m} / \mathrm{s}$ (12). Although the weight loss was comparable, the effect on arterial stiffness was more pronounced than that in our study. It is hard to find a plausible explanation for this large difference, particularly in view of the higher level of physical activity in our ILI group. However, direct comparison between the studies is difficult given by differences in study design, time of follow up, and various degree of obesity among the participants.

\section{Arterial stiffness and insulin sensitivity and biological age}

Arterial stiffness has been linked to age-related decline in muscle mass $(31,32)$. Loss of muscle mass is associated with reduced insu- lin sensitivity (33), and decreased insulin sensitivity is linked to arterial stiffness $(34,35)$. Individuals in the ILI group conserved more skeletal muscle mass than those in the LCD group. However, the greater loss of muscle mass in the LCD group compared to the ILI group did not translate into significant differences in terms of improvements in insulin sensitivity. The current popular notion that, in obese patients, a hypocaloric diet alone can reduce arterial stiffness could not be verified in Norwegian morbidly obese patients as the short-term LCD used failed to show any significant effect on arterial stiffness. Accordingly, the improved results in the ILI group are more likely to be mediated by the physical activity undertaken by individuals in that group.

Arterial stiffness is regarded as a measure of premature vascular ageing. According to the arterial stiffness reference value (36), the baseline biologic vascular age of our study population may be estimated to be 10-12 years older than it would be in normal-weight healthy individuals of a similar age. The reduction in PWV observed in the ILI group may translate into a reversal of age-related arterial stiffening of 5 to 7 years (37). Our findings support the notion that in morbidly obese individuals caloric restriction and physical activity might be preferable to weight loss by a LCD alone. Whether the short-term decline in PWV translates into a long-term clinically significant improvement in cardiovascular risk remains to be seen.

There are some limitations within the present study, which should be acknowledged. The major weaknesses are the lack of randomization (a)

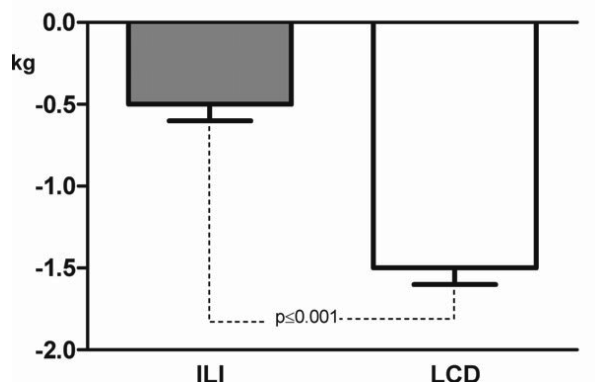

(b)

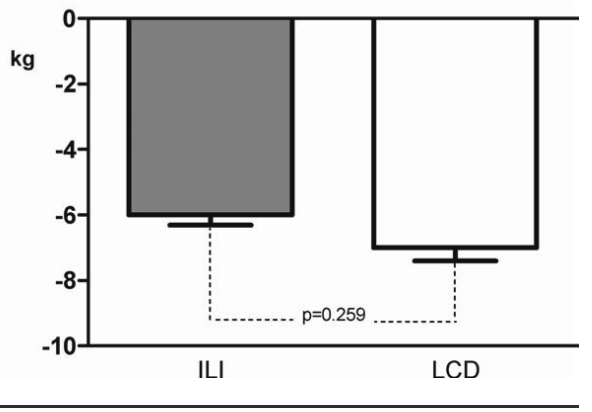

FIGURE 4 The loss of skeletal muscle mass and fat mass after 7 weeks of follow-up. 
TABLE 3 The change in weight, anthropometric measurements, glucose, and lipid metabolism after 7 weeks of ILI or LCD

\begin{tabular}{|c|c|c|c|c|}
\hline & ILI group & LCD group & Adjusted difference between groups & $P$ value \\
\hline Weight, kg & $-6.6(3.5)^{\dagger}$ & $-9.4(4.3)^{\dagger}$ & $1.5(0.5,2.9)$ & 0.006 \\
\hline $\mathrm{BMI}, \mathrm{kg} / \mathrm{m}^{2}$ & $-2.2(1.4)^{\dagger}$ & $-3.1(1.3)^{\dagger}$ & $0.6(0.2,0.9)$ & 0.003 \\
\hline $\begin{array}{l}\text { Waist circumference, } \mathrm{cm} \\
\text { Glucose metabolism }\end{array}$ & $-6(4)^{\dagger}$ & $-7(4)^{\dagger}$ & $0.5(-0.8,1.8)$ & 0.471 \\
\hline$\overline{\text { Insulin, pmol/L }}$ & $-35.9(37.1)^{\dagger}$ & $-43.4(82.1)^{\dagger}$ & $9.3(-0.6,19.1)$ & 0.065 \\
\hline Glucose, mmol/L & $-0.5(1.3)^{\dagger}$ & $-0.5(1.5)^{\dagger}$ & $0.0(-0.0,0.0)$ & 0.629 \\
\hline $\mathrm{HbA1C}, \%$ & $-0.4(0.5)^{\dagger}$ & $-0.4(0.6)^{\dagger}$ & $0.0(-0.1,0.1)$ & 0.894 \\
\hline HOMA-IR & $-1.8(2.0)^{\dagger}$ & $-2.5(4.7)^{\dagger}$ & $0.4(-0.05,0.9)$ & 0.083 \\
\hline \multicolumn{5}{|l|}{ Lipid metabolism } \\
\hline Total cholesterol, $\mathrm{mmol} / \mathrm{L}$ & $-0.6(0.6)^{\dagger}$ & $-0.4(0.6)^{\dagger}$ & $-0.2(-0.4,0.0)$ & 0.032 \\
\hline LDL cholesterol, mmol/L & $-0.3(0.6)^{\dagger}$ & $-0.2(0.5)^{*}$ & $-0.1(-0.3,0.1)$ & 0.193 \\
\hline HDL cholesterol, mmol/L & $-0.1(0.2)^{\star}$ & $-0.1(0.2)^{\dagger}$ & $0.1(0.0,0.1)$ & 0.007 \\
\hline Triglycerides, mmol/L & $-0.5(0.8)^{\dagger}$ & $-0.3(0.7)^{\dagger}$ & $-0.2(-0.3,-0.0)$ & 0.009 \\
\hline
\end{tabular}

Mean (SD or 95\% Cl).

${ }^{\star} P \leq 0.050$,

${ }^{\dagger} P \leq 0.001$. Paired samples $t$-test and ANCOVA adjusting for age, gender and baseline value of dependent variable.

and short follow-up time. According to Norwegian guidelines (38), tertiary care centers are obliged to provide morbidly obese patients with an appropriate treatment choice, either conservative or surgical. Both treatment choices are publicly funded. Accordingly, we found it unethical to assign patients to bariatric surgery if they both preferred and qualified for a conservative lifestyle intervention program, and vice versa. Other limitations include the lack of data illustrating the patients' adherence to the prescribed calorie restriction in both groups, and physical activity in the LCD group, and the absence of a weight maintenance period before the follow-up examination.

In summary, the findings from the present study indicate that a moderate caloric restriction combined with aerobic physical exercise can significantly reduce arterial stiffness in morbidly obese individuals after only 7 weeks of intervention. A LCD proved to be less effective in reducing arterial stiffness and showed no significant effect in spite of a clinically significant weight loss. It did, however, have a beneficial effect on both glucose and lipid metabolism, highlighting that important cardiovascular risk factors can be rapidly modified in morbidly obese individuals through a LCD. 0

\section{Acknowledgments}

We thank the following employees at the Morbid Obesity Center at Vestfold Hospital Trust: Heidi Omre Fon, Berit Mossing Bjørkås, and Linda Mathisen for their assistance with sampling and logistics, and Matthew McGee for proofreading the manuscript.

(C) 2012 The Obesity Society

\section{References}

1. Finucane MM, Stevens GA, Cowan MJ, et al. National, regional, and global trends in body-mass index since 1980: systematic analysis of health examination surveys and epidemiological studies with 960 country-years and 9.1 million participants. Lancet 2011;377:557-567.
2. Melanson KJ, McInnis KJ, Rippe JM, Blackburn G, Wilson PF. Obesity and cardiovascular disease risk: research update. [Review] [50 refs]. Cardiol Rev 2001;9: 202-207.

3. Flegal KM, Carroll MD, Kit BK, Ogden CL. Prevalence of obesity and trends in the distribution of body mass index among US adults, 1999-2010. JAMA 2012;307: 491-497.

4. Freedman DS, Khan LK, Serdula MK, Galuska DA, Dietz WH. Trends and correlates of class 3 obesity in the United States from 1990 through 2000. JAMA 2002; 288:1758-1761.

5. Hall KD, Sacks G, Chandramohan D, et al. Quantification of the effect of energy imbalance on bodyweight. Lancet 2011;378:826-837.

6. McAuley PA, Blair SN. Obesity paradoxes. J Sports Sci 2011;29:773-782.

7. Fogelholm M. Physical activity, fitness and fatness: relations to mortality, morbidity and disease risk factors. A systematic review. Obes Rev 2010;11:202-221.

8. Laurent S, Boutouyrie P, Asmar R, et al. Aortic stiffness is an independent predictor of all-cause and cardiovascular mortality in hypertensive patients. Hypertension 2001;37:1236-1241.

9. Vlachopoulos C, Aznaouridis K, Stefanadis C. Prediction of cardiovascular events and all-cause mortality with arterial stiffness: a systematic review and meta-analysis. [Review] [49 refs]. J Am Coll Cardiol 2010;55:1318-1327.

10. Lim HE, Park CG, Shin SH, Ahn JC, Seo HS, Oh DJ. Aortic pulse wave velocity as an independent marker of coronary artery disease. Blood Press 2004;13:369-375.

11. Wildman RP, Farhat GN, Patel AS, et al. Weight change is associated with change in arterial stiffness among healthy young adults. Hypertension 2005;45:187-192.

12. Dengo AL, Dennis EA, Orr JS, et al. Arterial destiffening with weight loss in overweight and obese middle-aged and older adults. Hypertension 2010;55:855-861.

13. Rider OJ, Francis JM, Ali MK, et al. Beneficial cardiovascular effects of bariatric surgical and dietary weight loss in obesity. J Am Coll Cardiol 2009;54:718-726.

14. Wildman RP, Mackey RH, Bostom A, Thompson T, Sutton-Tyrrell K. Measures of obesity are associated with vascular stiffness in young and older adults. Hypertension 2003;42:468-473.

15. Vaitkevicius PV, Fleg JL, Engel JH, et al. Effects of age and aerobic capacity on arterial stiffness in healthy adults. Circulation 1993;88(4 Pt 1):1456-1462.

16. Tanaka H, Safar ME. Influence of lifestyle modification on arterial stiffness and wave reflections. Am J Hypertens 2005; 18:137-144.

17. Mansia G, De BG, Dominiczak A, et al. 2007 ESH-ESC Guidelines for the management of arterial hypertension: the task force for the management of arterial hypertension of the European Society of Hypertension (ESH) and of the European Society of Cardiology (ESC). Blood Press 2007;16:135-232.

18. Laurent S, Cockcroft J, Van BL, et al. Expert consensus document on arterial stiffness: methodological issues and clinical applications. Eur Heart $J$ 2006;27: 2588-2605.

19. Nordstrand N, Gjevestad E, Dinh KN, et al. The relationship between various measures of obesity and arterial stiffness in morbidly obese patients. BMC Cardiovasc Disord 2011;11:7.

20. World Medical Association declaration of Helsinki. Recommendations guiding physicians in biomedical research involving human subjects. JAMA 1997;277: 925-926. 
21. Schofield WN. Predicting basal metabolic rate, new standards and review of previous work. Hum Nutr Clin Nutr 1985;39 Suppl 1:5-41.

22. Ainsworth BE, Haskell WL, Herrman SD. Compendium of Physical Activities: second update of codes and MET values. Med Sci Sports Exerc 2011;43:1575-1581.

23. Nasjonalt råd for ernæring. Kostråd for å fremme folkehelsen og forebygge kroniske sykdommer i Norge. Helsedirektoratet. 2011.

24. Ainsworth BE, Haskell WL, Leon AS, et al. Compendium of physical activities: classification of energy costs of human physical activities. Med Sci Sports Exerc 1993;25:71-80.

25. Rubak S, Sandbaek A, Lauritzen T, Christensen B. Motivational interviewing: a systematic review and meta-analysis. Br J Gen Pract 2005;55:305-312.

26. Matthews DR, Hosker JP, Rudenski AS, Naylor BA, Treacher DF, Turner RC. Homeostasis model assessment: insulin resistance and beta-cell function from fasting plasma glucose and insulin concentrations in man. Diabetologia 1985;28:412-419.

27. Friedewald WT, Levy RI, Fredrickson DS. Estimation of the concentration of lowdensity lipoprotein cholesterol in plasma, without use of the preparative ultracentrifuge. Clin Chem 1972;18:499-502.

28. Barinas-Mitchell E, Kuller LH, Sutton-Tyrrell K, et al. Effect of weight loss an nutritional intervention on arterial stiffness in type 2 diabetes. Diabetes Care 2006 29:2218-2222

29. McNamee R. Confounding and confounders. Occup Environ Med 164; 60:227-234.

30. Blumenthal JA, Babyak MA, Hinderliter A, et al. Effects of the DASH diet alone and in combination with exercise and weight loss on blood pressure and cardiovas- cular biomarkers in men and women with high blood pressure: the ENCORE study. Arch Intern Med 2010;170:126-135.

31. Abbatecola AM, Chiodini P, Gallo C, et al. Pulse wave velocity is associated with muscle mass decline: Health ABC study. Age (Dordr) 2011;34:469-478.

32. Ochi M, Kohara $\mathrm{K}$, Tabara $\mathrm{Y}$, et al Arterial stiffness is associated with low thigh muscle mass in middle-aged to elderly men. Atherosclerosis 2010;212: 327-332.

33. Volpi E, Nazemi R, Fujita S. Muscle tissue changes with aging. Curr Opin Clin Nutr Metab Care 2004; 7:405-410.

34. Stehouwer CD, Henry RM, Ferreira I. Arterial stiffness in diabetes and the metabolic syndrome: a pathway to cardiovascular disease. Diabetologia 2008;51: 527-539.

35. Ferreira I, Boreham CA, Twisk JW, et al. Clustering of metabolic syndrome risk factors and arterial stiffness in young adults: the Northern Ireland Young Hearts Project. J Hypertens 2007;25:1009-1020

36. Determinants of pulse wave velocity in healthy people and in the presence of cardiovascular risk factors: 'establishing normal and reference values'. Eur Heart J 2010;31:2338-2350.

37. Avolio AP, Chen SG, Wang RP, Zhang CL, Li MF, O'Rourke MF. Effects of aging on changing arterial compliance and left ventricular load in a northern Chinese urban community. Circulation 1983;68:50-58.

38. Jakobsen GS, Hofso D, Roislien J, Sandbu R, Hjelmesaeth J. Morbidly obese patients--who undergoes bariatric surgery? Obes Surg 2010;20:1142-1148. 


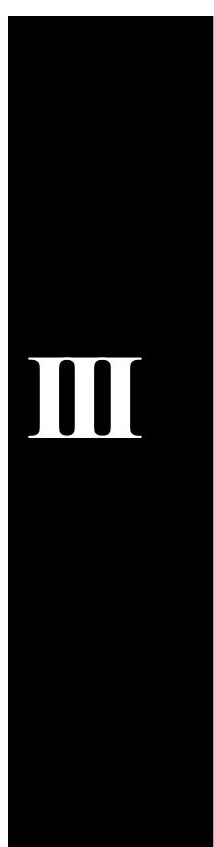





\title{
Effects of Intensive Lifestyle Intervention and Gastric Bypass on Aortic Stiffness: A 1-Year Nonrandomized Clinical Study
}

\author{
Espen Gjevestad ${ }^{1,2}$, Jøran Hjelmesath ${ }^{1,3}$, Rune Sandbu ${ }^{1}$ and Njord Nordstrand ${ }^{1}$
}

Objective: To compare the long-term effects of Roux-en-Y gastric bypass (GBS) and intensive lifestyle intervention (ILI) on aortic stiffness.

Methods: Nonrandomized clinical trial. Aortic stiffness was assessed by carotid-femoral pulse wave velocity ( ${ }_{\mathrm{c}} \mathrm{PWV}$ ) using high-fidelity applanation tonometry.

Results: A total of 159 treatment seeking morbidly obese patients were included, 82 (54 females) in the GBS-group and 77 (48 females) in the ILI-group. Participants in the GBS-group were younger $(42.0 \pm 9.9$ vs. $46.4 \pm 10.5$ years), heavier (BMl $45.7 \pm 5.3$ vs. $42.0 \pm 4.9 \mathrm{~kg} / \mathrm{m}^{2}$ ), and had lower systolic pressure (137 \pm 19 vs. $145 \pm 18 \mathrm{~mm} \mathrm{Hg}$ ) and pulse pressure ( $57 \pm 16$ vs. $65 \pm 17 \mathrm{~mm} \mathrm{Hg}$ ), all $P \leq 0.006$. Mean (SD) ${ }_{\text {cf } P W V}$ at baseline was $8.6 \pm 1.7 \mathrm{~m} / \mathrm{s}$ in the GBS-group and $8.6 \pm 1.9 \mathrm{~m} / \mathrm{s}$ in the ILI-group, $P=0.959$. At follow-up, mean $(95 \% \mathrm{Cl})$ weight loss was larger in the GBS-group $-43.3(-46.0$ to -40.7$)$ vs. $-12.1(-14.6$ to -9.6$) \mathrm{kg}$ than in the ILI-group, $P<0.001$. The mean change in ${ }_{\text {cf }} P W V$ was $-0.02(-0.31$ to 0.27$) \mathrm{m} / \mathrm{s}$ in the GBS-group and $0.03(-0.28$ to 0.33$) \mathrm{m} / \mathrm{s}$ in the ILI-group, both $P \geq 0.412$; adjusted between-group difference (ANCOVA) $0.05(-0.40$ to 0.49$) \mathrm{m} / \mathrm{s}, P=0.836$. The adjusted regression analysis showed that weight loss was associated with increased ${ }_{\mathrm{cf}} \mathrm{PWV}$ in the GBS-group.

Conclusion: GBS and ILI had no significant long-term effects on aortic stiffness in treatment-seeking morbidly obese individuals.

Obesity (2014) 00, 00-00. doi:10.1002/oby.20880

\section{Introduction}

Obesity is a global health concern of epidemic proportions (1) and is associated with increased risk of diabetes, obstructive sleep apnea, cardiovascular disease (CVD), some cancer types and premature death (2). The cornerstone of obesity management is weight loss, and both dietary and surgically induced weight loss improve the CVD risk profile in terms of reduced blood pressure, blood glucose, and lipid levels in morbidly obese individuals (3). In addition, increased aerobic fitness and physical activity improve CVD risk independent of weight loss, especially in unfit overweight and obese individuals (4). CVD risk assessment in obese individuals is largely based on established risk estimates such as the Framingham Risk Score which emphasizes smoking habits, lipid levels, and blood pressure (5).

Aortic stiffness provides additional information regarding CVD risk beyond traditional risk factors (6). Further, unlike fluctuating risk factors like blood lipids, glucose, and hypertension, aortic stiffness represents the accumulated damaging effects of all these measures over time (7). Aortic stiffness is largely determined by structural changes in the arterial wall such as (1) increased collagen-elastin ratio, (2) fractures of elastic lamella and the cross-linking of collagen, and (3) increased amount of advanced glycation end-products. Structural changes in aorta occur slowly over a period of years (8) while other factors like adrenergic activity and aerobe exercise may modulate aortic compliance over a short space of time $(9,10)$. Aortic stiffness is considered a valid surrogate end point of CVD (7) and is also an independent predictor of all-cause mortality (7) and cardiovascular morbidity and mortality $(7,11,12)$. Carotid to femoral pulse wave velocity $\left({ }_{c f} \mathrm{PWV}\right)$ has been recommended as the gold-standard measure of aortic stiffness $(13,14)$.

Some short-term ( $<12$ months) studies have shown reduced aortic stiffness after various lifestyle intervention programs in normal weight (15), overweight (16), obese (17), and morbidly obese patients (18). Similarly, some long-term ( $\geq 1$-year) studies have demonstrated beneficial effects of weight loss on aortic stiffness in metabolically healthy obese patients after diet alone (19), diet and physical activity (20), and diet or bariatric surgery (21). In addition, one study demonstrated reduced ${ }_{\text {ff }} \mathrm{PWV}$ after weight loss in obese diabetic patients (22). However, the comparative effect of bariatric

\footnotetext{
${ }^{1}$ Morbid Obesity Center, Vestfold Hospital Trust, Tønsberg, Norway ${ }^{2}$ Clinic Physical Medicine and Rehabilitation, Vestfold Hospital Trust, Stavern, Norway. ${ }^{3}$ Department of Endocrinology, Morbid Obesity and Preventive Medicine, Institute of Clinical Medicine, University of Oslo, Norway. Correspondence: Jøran Hjelmesæth (joran.hjelmeseth@siv.no).
} 


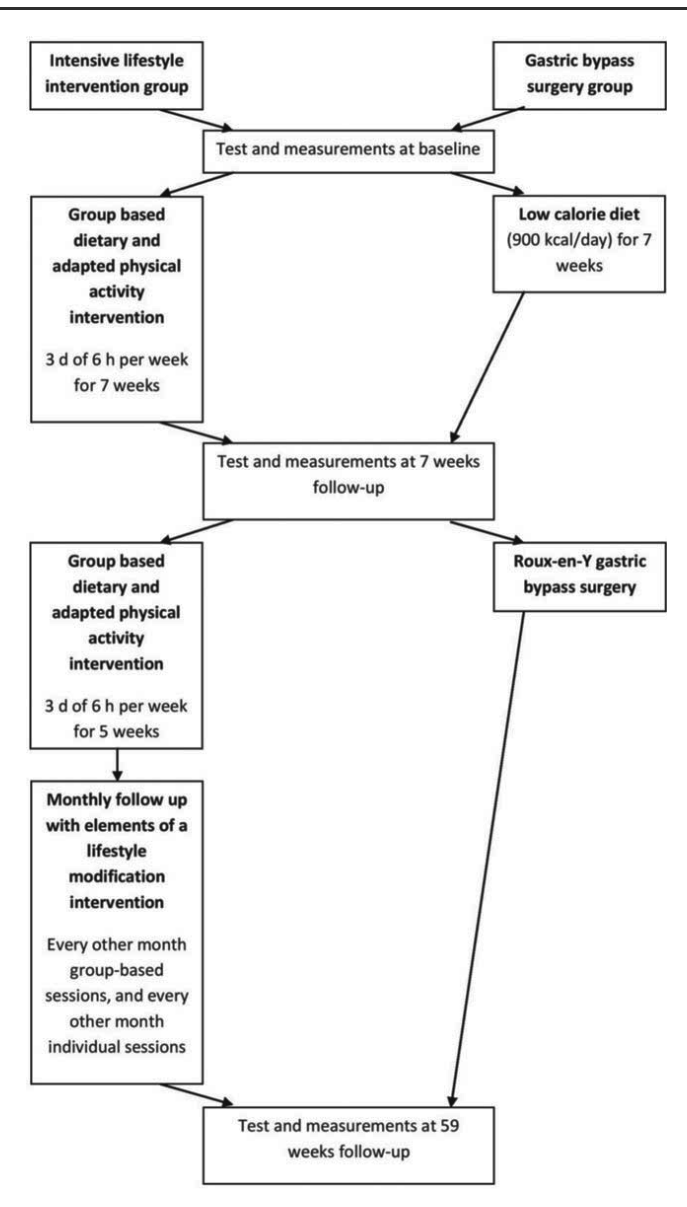

Figure 1 Flow chart of interventions.

surgery and intensive lifestyle intervention (ILI) on aortic stiffness remains unknown.

The primary aim of this study was to compare the 1-year effect of an ILI program and a surgical weight loss program on ${ }_{\text {cf }} \mathrm{PWV}$ in morbidly obese individuals. Our main hypothesis was that patients who underwent Roux-en-Y gastric bypass surgery (GBS) after an initial low calorie diet would have a larger mean 1-year reduction of ${ }_{c f} \mathrm{PWV}$ compared to those who underwent an ILI program.

\section{Methods}

\section{Study design}

This is the second part of a nonrandomized clinical trial comparing the effects of an ILI and an initial 7-week low-calorie diet (LCD) followed by GBS on aortic stiffness in treatment seeking morbidly obese patients (Clinical Trials.gov Identifier NCT00626964). Preliminary results from baseline (23), and short-term results after 7 weeks (18) are published elsewhere. The protocol was approved by the regional ethics committee of the Southern Norway Regional Health Authority (identifier S-05175). All procedures were conducted according to the principles expressed in the Declaration of Helsinki (24). Written informed consent was provided by all participants.

\section{Setting}

The present study was conducted at the Morbid Obesity Center, Vestfold Hospital Trust, from February 2008 to June 2012. Before inclusion, patients were either assigned to an ILI program at the Clinic of Physical Medicine and Rehabilitation (CPMR) or to an initial LCD followed by GBS at the Morbid Obesity Center (MOC).

\section{Participants}

All participants were recruited from our tertiary care center. For practical reasons and to maximize compliance, participants had to reside within $100 \mathrm{~km}$ of either the CPMR or MOC. The patients in the ILI-group were all selected from patients that had registered for participation in a standardized health promotion and weight reduction program at the CPMR. Participants in the GBS-group were selected from patients as a result of undergoing bariatric surgery at our hospital. The decision regarding the type of intervention to be undertaken was made prior to inclusion to the present study and was not part of the protocol.

Inclusion criteria were $\mathrm{BMI} \geq 35 \mathrm{~kg} / \mathrm{m}^{2}$ with at least one obesityrelated comorbidity, or a BMI $\geq 40 \mathrm{~kg} / \mathrm{m}^{2}$. Patients were excluded if they had uncompensated heart failure, cardiac arrhythmias, unstable angina, end-stage renal disease, bleeding disturbances, serious psychiatric disorders, serious eating disorders, cardiac pacemakers, intra-cardiac devices, or a recent cerebrovascular event or myocardial infarction (within the past 6 months).

\section{Interventions}

The first 7 weeks of ILI is described in detail elsewhere (18). Briefly, the ILI included both a dietary and physical intervention. The first year of the intervention was divided into two stages (Figure 1). The first 12 weeks included treatment sessions 3 days per week, with patients participating in two supervised training sessions for 60-90 min and lectures on nutrition, physical activity, and motivation each day. Patients received a dietary plan with an energy restriction of $1000 \mathrm{kcal} / \mathrm{day}$ of the calculated total energy expenditure at baseline (25). Study participants had individual sessions with qualified personnel who used elements of a lifestyle modification intervention in order to invoke behavioral change in the participants. During weeks 13-52 patients received monthly follow-up, alternating between group based and individual sessions every other month. Patients were advised to maintain physical activity for $60-90 \mathrm{~min}$ per day in order to increase or maintain weight loss (26), and during the individual sessions patients were told to describe their physical activity level.

Preceding surgery, patients in the GBS-group completed a 7 weeks LCD (<900 kcal per day; Figure 1). Laparoscopic Roux-en-Y GBS were performed in 94 patients. The gastric pouch was about $25 \mathrm{ml}$, 


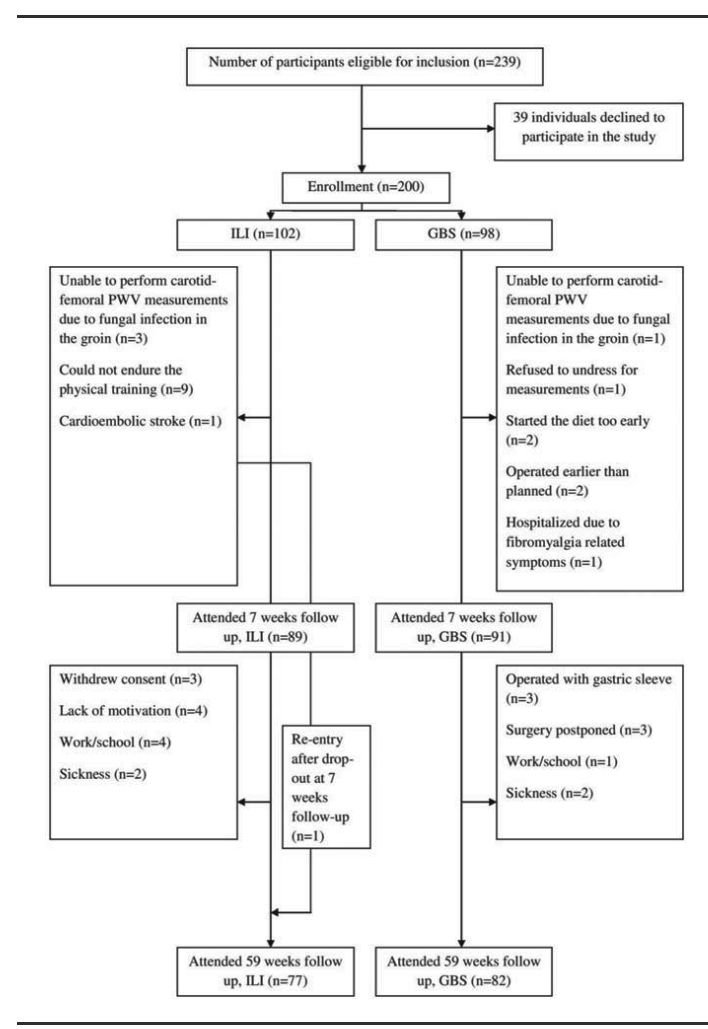

Figure 2 Flow chart.

while the intestinal limb lengths were measured as follows: alimentary limb $120 \mathrm{~cm}$, biliopancreatic limb $60 \mathrm{~cm}$, and common channel, variable length. A standardized regimen of dietary supplements and a proton pump inhibitor were prescribed to all patients after surgery.

\section{Outcomes}

The main outcome variable was the 1-year change in aortic stiffness measured by ${ }_{\text {ff }} \mathrm{PWV}$. Secondary outcome variables were changes in blood pressure, body weight, and body composition.

\section{Data source and measurements}

Detailed descriptions of data sources and measurements are published elsewhere (18). Briefly, all participants underwent medical examinations which included collection of blood samples, measurement of body weight, height, and waist circumference, and analysis of body composition using bioelectrical impedance analysis (Inbody 720, Body Composition Analyzer, Biospace, Seol, South Korea). Resting blood pressure was measured using an electronic blood pressure recorder with an appropriately sized cuff (Dinamap ${ }^{\circledR}$, ProCare Series, G.E. Medical Systems, Buckinghamshire, UK) with the patient sitting in an upright position. Arterial hypertension was defined by either a systolic blood pressure $\geq 140 \mathrm{~mm} \mathrm{Hg}$, diastolic blood pressure $\geq 90 \mathrm{~mm} \mathrm{Hg}$ or the use of antihypertensive medication. Ischemic heart disease was defined as a history of stable coronary artery disease, percutaneous coronary intervention, coronary artery bypass graft surgery, or myocardial infarction. Mean arterial pressure (MAP) was calculated as [(diastolic pressure x 2) + systolic pressure]/ 3 (27). Type 2 diabetes was diagnosed in patients who had a prior history of type 2 diabetes or a fasting serum glucose level $\geq 7.0$ $\mathrm{mmol} / \mathrm{L}$ (28). Model Assessment Insulin Resistance (HOMA-IR) was calculated as [fasting serum glucose $(\mathrm{mmol} / \mathrm{L}) \times$ fasting serum insulin (pmol/L)]/135 (29) and low-density lipoprotein cholesterol (LDL) concentrations were estimated using the Friedewald equation (30).

The SphygmoCor ${ }^{\circledR}$ system (AtCor Medical, Sidney, Australia) and a single high-fidelity applanation tonometer $\left(\right.$ Millar $^{\mathbb{B}}$ ) were used to measure ${ }_{c f} \mathrm{PWV}$. Pulse waves were obtained sequentially from the carotid and femoral artery. The travel distance was calculated by subtracting the distance between the carotid artery and the sternal notch from the distance between the sternal notch and the femoral artery (13). The subtracted travel distance measured at baseline was used in the calculations of ${ }_{\text {cf }} \mathrm{PWV}$ at follow-up.

Physical activity level was measured with a self reported questionnaire (CONOR) (31) at 59 weeks follow-up. The questionnaire divides physical activity into two main parts: leisure time physical activity and occupational physical activity. Physical activity during leisure time was further divided into light physical activity (not sweaty and breathless) and vigorous physical activity (sweaty and breathless), and scored according to duration (hour/week) (none, $<1,1-2,>3$ ). Occupational physical activity was scored according to type of work (mainly sedentary, mainly walking, mainly walking or/and carrying, heavy manual labor).

\section{Laboratory analyses}

Analyses of serum glucose and blood lipids were performed using dry reagent slide technology on the Vitros FS 5.1 (Ortho-Clinical Diagnostics, NY, USA). Glycated hemoglobin (HbA1c) was analyzed using high performance liquid chromatography on Tosoh HLC-723 G7 (Tosoh Corporation, Tokyo, Japan). Sera for analysis of insulin were stored at $-20^{\circ} \mathrm{C}$ and analyzed within one week of blood sampling (Linco Research, St. Charles, MO).

\section{Sample size}

The sample size was calculated based on a publication demonstrating a mean reduction of $1.4 \mathrm{~m} / \mathrm{s}$ in ${ }_{\mathrm{cf}} \mathrm{PWV}$ after $8 \%$ nonsurgical weight loss (22). We anticipated a mean 1 year weight loss of $8 \%$ and $30 \%$, and a mean \pm SD reduction in ${ }_{\mathrm{cf}} \mathrm{PWV}$ of $1.4 \pm 2.8$ and $2.8 \pm 2.8 \mathrm{~m} / \mathrm{s}$, in the lifestyle and surgery groups, respectively. To show a statistically significant difference between the two groups at a power of $80 \%(\alpha=0.05)$, at least 126 individuals (63 in each group) had to complete the study. To allow for a drop-out rate of $<40 \%$ it was necessary to include 200 patients (100 in each group).

\section{Statistical methods}

Data are presented as mean \pm SD or number (\%) unless otherwise specified. Differences between groups at baseline were analyzed using independent samples $t$-tests for continuous variables and Fisher's exact tests for categorical variables. Within-group changes from baseline to follow-up were analyzed using paired samples $t$-tests for continuous data and McNemar's test for categorical data. The 
TABLE 1 Baseline characteristics, body composition, and cardiovascular risk factors among 159 morbidly obese patients who completed the study according to treatment choice

\begin{tabular}{|c|c|c|c|}
\hline & $\begin{array}{l}\text { Intensive lifestyle } \\
\text { intervention }(n=77)\end{array}$ & $\begin{array}{l}\text { Gastric bypass } \\
\text { surgery }(n=82)\end{array}$ & $P$-value \\
\hline Age (years) & $46.4 \pm 10.5$ & $42.0 \pm 9.9$ & 0.006 \\
\hline Gender, female (\%) & $48(62)$ & $54(66)$ & 0.741 \\
\hline Diabetes $(\%)$ & $17(22)$ & $24(29)$ & 0.365 \\
\hline Hypertension (\%) & $59(78)$ & $50(61)$ & 0.026 \\
\hline Ischemic heart disease (\%) & $12(16)$ & $2(2)$ & 0.004 \\
\hline Smokers (\%) & $8(10)$ & $17(21)$ & 0.084 \\
\hline \multicolumn{4}{|l|}{ Weight and anthropometric measures } \\
\hline Weight $(\mathrm{kg})$ & $124 \pm 20$ & $137 \pm 22$ & $<0.001$ \\
\hline Body mass index $\left(\mathrm{kg} / \mathrm{m}^{2}\right)$ & $42.0 \pm 4.9$ & $45.7 \pm 5.3$ & $<0.001$ \\
\hline Waist circumference (cm) & $126 \pm 12$ & $132 \pm 12$ & 0.001 \\
\hline Fat mass $(\mathrm{kg})$ & $57.6 \pm 12.1$ & $66.5 \pm 12.5$ & $<0.001$ \\
\hline Skeletal muscle mass $(\mathrm{kg})$ & $37.5 \pm 7.2$ & $39.4 \pm 8.1$ & 0.120 \\
\hline \multicolumn{4}{|l|}{${ }_{c f} P W V$ and blood pressure } \\
\hline Pulse wave velocity (carotid femoral) $(\mathrm{m} / \mathrm{s})$ & $8.6 \pm 1.9$ & $8.6 \pm 1.7$ & 0.959 \\
\hline Systolic blood pressure $(\mathrm{mm} \mathrm{Hg})$ & $145 \pm 18$ & $137 \pm 19$ & 0.006 \\
\hline Diastolic blood pressure (mm Hg) & $81 \pm 11$ & $79 \pm 13$ & 0.523 \\
\hline Pulse pressure $(\mathrm{mm} \mathrm{Hg})$ & $65 \pm 17$ & $57 \pm 16$ & 0.002 \\
\hline Mean arterial pressure $(\mathrm{mm} \mathrm{Hg})$ & $102 \pm 11$ & $99 \pm 14$ & 0.074 \\
\hline Heart rate (beats/min) & $79 \pm 13$ & $77 \pm 14$ & 0.288 \\
\hline \multicolumn{4}{|l|}{ Glucose and lipid metabolism } \\
\hline Insulin $(\mathrm{pmol} / \mathrm{L})^{\dagger}$ & $87(48,115)$ & $93(58,140)$ & 0.144 \\
\hline Glucose $(\mathrm{mmol} / \mathrm{L})^{\dagger}$ & $5.3(4.9,6.4)$ & $5.4(5.16 .4)$ & 0.248 \\
\hline $\mathrm{HbA1c}(\%)^{\dagger}$ & $5.7(5.4,6.1)$ & $5.8(5.3,6.3)$ & 0.772 \\
\hline HOMA-IR ${ }^{\dagger}$ & $3.4(1.9,5.7)$ & $4.1(2.3,6.2)$ & 0.157 \\
\hline Total cholesterol (mmol/L) & $5.1 \pm 1.0$ & $4.9 \pm 1.0$ & 0.287 \\
\hline LDL-cholesterol (mmol/L) & $3.1 \pm 1.0$ & $3.1 \pm 0.9$ & 0.936 \\
\hline HDL-cholesterol $(\mathrm{mmol} / \mathrm{L})^{\dagger}$ & $1.2(1.0,1.4)$ & $1.1(0.9,1.3)$ & 0.072 \\
\hline Triglycerides $(\mathrm{mmol} / \mathrm{L})^{\dagger}$ & $1.4(1.1,2.1)$ & $1.5(1.0,2.0)$ & 0.820 \\
\hline \multicolumn{4}{|l|}{ Drugs } \\
\hline Statins $(\%)$ & $18(23)$ & $12(15)$ & 0.223 \\
\hline Any antihypertensive drugs (\%) & $35(46)$ & $29(35)$ & 0.201 \\
\hline Beta blocker (\%) & $14(18)$ & $10(12)$ & 0.376 \\
\hline Calcium channel blocker (\%) & $12(16)$ & $12(12)$ & 0.647 \\
\hline Renin-angiotensin-aldosteron-system inhibitor (\%) & $28(36)$ & $23(28)$ & 0.309 \\
\hline Diuretics $(\%)$ & $18(23)$ & $15(18)$ & 0.442 \\
\hline Other blood pressure medications (\%) & $6(8)$ & $4(5)$ & 0.525 \\
\hline Any antidiabetic/ glucose lowering drugs (\%) & $18(23)$ & $19(23)$ & 0.999 \\
\hline Glitazone (\%) & $2(3)$ & $5(6)$ & 0.444 \\
\hline Sulfonylurea (\%) & $5(7)$ & $8(10)$ & 0.567 \\
\hline Insulin (\%) & $4(5)$ & $1(1)$ & 0.999 \\
\hline Dipeptidyl peptidase-4 inhibitor (\%) & 0 & $1(1)$ & 0.999 \\
\hline Metformin (\%) & $12(16)$ & $16(20)$ & 0.540 \\
\hline
\end{tabular}

Values reported are number (\%) or mean \pm SD unless otherwise noted. Independent sample $t$-test was used for normally distributed data and Mann-Whitney for nonparametric data. Fischer's exact test was used for categorical data. ${ }^{\dagger}$ Reported as Median (Q1, Q3) as a result of skewed distribution.

potential interaction between gender and treatment choice was analyzed using two-way analysis of covariance. The difference in ${ }_{\mathrm{cf}} \mathrm{PWV}$ between groups at 59-weeks follow-up were analyzed using one-way analysis of covariance (ANCOVA) including age, gender, history of coronary artery disease, baseline BMI, MAP, heart rate, and ${ }_{\mathrm{cf}} \mathrm{PWV}$ as covariates. Differences in systolic blood pressure, diastolic blood pressure, and pulse pressure at follow-up were analyzed using ANCOVA including age, gender, baseline BMI, history of 


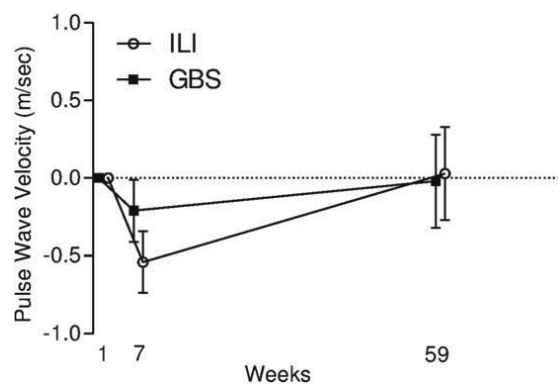

Figure 3 Short- and long-term mean adjusted changes in aortic carotid femoral pulse wave velocity $\left(_{c \mathrm{P}} \mathrm{PW}\right)(\mathrm{m} / \mathrm{s}, 95 \% \mathrm{Cl})$ in the morbidly obese patients who underwent intensive lifestyle intervention (ILI) or Roux-en-Y gastric bypass surgery (GBS).

coronary artery disease, and baseline status in dependent variable as covariates.

In addition, we performed post hoc subgroup correlation analyses between changes in anthropometric variables and changes in ${ }_{\mathrm{cf}} \mathrm{PWV}$ in the two treatment groups. Finally, multiple linear regression analyses with ${ }_{\text {cf }} \mathrm{PWV}$-changes as effect variable and age, gender, history of coronary artery disease, and baseline values of BMI, mean arterial pressure, heart rate, ${ }_{\mathrm{cf}} \mathrm{PWV}$, and anthropometric parameters as explanatory variables were performed in each treatment group.

$P$-values below 0.05 were considered statistically significant. Particular attention should, however, be directed towards smaller $P$-values, i.e., those below 0.01 , because a considerable number of $P$-values have been calculated.

\section{Results}

A total of 239 individuals were eligible, 39 declined to participate, leaving 200 (64\% female) participants to be included in the study (Figure 2). During the first 7 weeks, 13 individuals in the ILI-group and seven individuals in the GBS-group withdrew. During the last period of 52 weeks an additional 13 patients in the ILI-group and nine patients in the GBS-group withdrew, while one patient re-entered the ILI-group at 59 weeks follow-up. This leaves 159 (64\% female) completers ( $80 \%$ of all included patients) in the present analysis, a total number of $77(76 \%)$ in the ILI-group and $82(84 \%)$ in the GBS-group.

The GBS-patients were approximately 4 years younger, $13 \mathrm{~kg}$ heavier, and had lower systolic blood pressure, pulse pressure, and a lower prevalence of hypertension and ischemic heart disease compared to the ILI-patients. The treatment groups did not differ significantly in terms of ${ }_{\mathrm{cf}} \mathrm{PWV}$ or in the use of anti-hypertensive drugs, lipid-lowering drugs, and antidiabetic drugs (Table 1).

\section{Changes in aortic stiffness, blood pressure and heart rate}

The average ${ }_{\mathrm{cf}} \mathrm{PWV}$ for completers in both groups at baseline, 7 and 59 weeks are shown in Figure 3. As previously reported (18), in the short-term (7-weeks) the ILI-group had a significantly larger mean $(95 \% \mathrm{CI})$ reduction of ${ }_{\mathrm{cf}} \mathrm{PWV}$ than the GBS-group (low calorie diet $) ;-0.6(-0.8,-0.4) \mathrm{m} / \mathrm{s}$ vs. $-0.2(-0.4,0.0) \mathrm{m} / \mathrm{s}$, adjusted between-group difference $0.4(0.1,0.6) \mathrm{m} / \mathrm{s}, P=0.004$. In contrast, there were no significant changes in ${ }_{\mathrm{cf}} \mathrm{PWV}$ from baseline to 59weeks in either treatment group, and no significant adjusted between group difference was found (Figure 3 and Table 2). No significant interaction between gender and treatment group was found $(P=0.158)$. Patients in the GBS-group had a significantly larger decline in heart rate compared to patients in the ILI-group and tended to have larger reductions of systolic and diastolic blood pressure (Table 2).

\section{Impact of weight loss according to treatment group}

Figure 4 illustrates that weight loss and reduced WC were associated with decreasing ${ }_{\text {cf }} \mathrm{PWV}$ in the ILI-group, but, in contrast, with increasing ${ }_{c f} \mathrm{PWV}$ in the GBS-group. After adjustments for age, gender, history of coronary artery disease, and baseline values of BMI, $\mathrm{MAP}$, heart rate, and ${ }_{\mathrm{cf}} \mathrm{PWV}$, multiple linear regression analyses showed that the associations between changes in ${ }_{c f} \mathrm{PWV}$ and changes in WC, BMI, and body weight remained statistically significant in the GBS-group only (all $P<0.006$ ).

\section{Weight loss, glucose metabolism, and lipids}

Patients in the GBS-group had, compared to patients in the ILIgroup, a larger percentage weight loss, $32 \%$ vs. $10 \%$, and greater reductions of mean waist circumference, fat mass, and skeletal muscle mass (Table 2).

Patients in the GBS-group also achieved larger reductions of serum insulin, glucose, HbA1c, HOMA-IR, total cholesterol, LDL cholesterol, and triglycerides, and a larger increase in mean HDL cholesterol compared to patients in the ILI-group (Table 2).

\section{Changes in medications}

The proportion of patients treated with any antihypertensive and antidiabetic drugs decreased significantly during the first 59 weeks in the surgery group only. Specifically, the number of GBS-patients treated with RAAS-inhibitors, beta blockers, and diuretics decreased from 23 to $13(44 \%), 10$ to $4(60 \%)$, and 15 to $7(53 \%)$, respectively, all $P<0.032$. The number of patients using sulfonylurea or metformin decreased from 8 to $0(100 \%)$ and 16 to $3(81 \%)$, $P=0.008$ and $P<0.001$, respectively. There was no significant change in the use of statins in either group, both $P>0.374$.

\section{Physical activity level at follow-up}

The majority of patients reported that they completed $>3$ hours/ week of light physical activity and $>3$ hours/week of vigorous physical activity at follow-up, with no significant differences between groups (Supporting Information Table 1).

\section{Discussion}

Our results did not confirm the hypothesis that patients undergoing bariatric surgery would achieve a larger mean 1-year reduction of 
TABLE 2 Within-group changes and adjusted between-group differences in carotid to femoral pulse wave velocity, blood pressure, heart rate, anthropometric measures, glucose metabolism, and lipids in 159 morbidly obese patients during the study

\begin{tabular}{|c|c|c|c|c|}
\hline & $\begin{array}{c}\text { Intensive lifestyle } \\
\text { intervention group }(n=77)\end{array}$ & $\begin{array}{l}\text { Gastric bypass surgery } \\
\text { group }(n=82)\end{array}$ & $\begin{array}{l}\text { Adjusted difference } \\
\text { between groups }\end{array}$ & $P$-value \\
\hline Pulse wave velocity (carotid femoral) $(\mathrm{m} / \mathrm{s})^{\mathrm{a}}$ & $0.03(-0.28$ to 0.33$)$ & $-0.02(-0.31$ to 0.27$)$ & $0.05(-0.40$ to 0.49$)$ & 0.836 \\
\hline Systolic blood pressure $(\mathrm{mm} \mathrm{Hg})^{\mathrm{a}}$ & $-2(-6$ to 3$)$ & $-8(-13 \text { to }-4)^{*}$ & 6 (0 to 13) & 0.057 \\
\hline Diastolic blood pressure $(\mathrm{mm} \mathrm{Hg})^{\mathrm{a}}$ & -1 ( -3 to 2$)$ & $-4(-6$ to -2$)$ & $3(0$ to 7$)$ & 0.067 \\
\hline Pulse Pressure $(\mathrm{mm} \mathrm{Hg})^{\mathrm{a}}$ & $0(-4$ to 4$)$ & $-5(-9 \text { to }-2)^{\star \star}$ & $5(0$ to 10$)$ & 0.074 \\
\hline Mean Arterial Pressure $(\mathrm{mm} \mathrm{Hg})^{a}$ & $-1(-4$ to 2$)$ & $-6(-8 \text { to }-3)^{*}$ & $4(0$ to 8$)$ & 0.038 \\
\hline Heart rate (beats $/ \mathrm{min})^{\mathrm{a}}$ & $-6(-8 \text { to }-3)^{\star \star}$ & $-11(-14 \text { to }-9)^{\star \star}$ & 7 (3 to 10$)$ & $<0.001$ \\
\hline Weight, $\mathrm{kg}$ & $-12.1(-14.6 \text { to }-9.6)^{\star \star}$ & $-43.3(-46.0 \text { to }-40.7)^{\star \star}$ & $27.6(24.0$ to 31.1$)$ & $<0.001$ \\
\hline $\mathrm{BMI}, \mathrm{kg} / \mathrm{m}^{2}$ & $-4.0(-4.9 \text { to }-3.2)^{\star \star}$ & $-14.4(-15.3 \text { to }-13.6)^{\star \star}$ & 9.3 (8.2 to 10.5$)$ & $<0.001$ \\
\hline Waist circumference, $\mathrm{cm}$ & $-10.9(-13.2 \text { to }-8.6)^{* *}$ & $-30.5(-32.9 \text { to }-28.0)^{\star *}$ & 17.1 (13.6 to 20.5$)$ & $<0.001$ \\
\hline Fat mass, $\mathrm{kg}$ & $-10.2(-12.5 \text { to }-7.9)^{* *}$ & $-36.6(-38.9 \text { to }-34.3)^{\star *}$ & 24.2 (20.9 to 27.5$)$ & $<0.001$ \\
\hline Skeletal muscle mass, $\mathrm{kg}$ & $-0.5(-1.5$ to 0.6$)$ & $-4.1(-5.2 \text { to }-3.1)^{\star \star}$ & $3.3(1.7$ to 4.9$)$ & $<0.001$ \\
\hline \multicolumn{5}{|l|}{ Glucose metabolism } \\
\hline Insulin, pmol/L & $-21(-34 \text { to }-8)^{*}$ & $-81(-102 \text { to }-59)^{\star \star}$ & 41 (28 to 55) & $<0.001$ \\
\hline Glucose, $\mathrm{mmol} / \mathrm{L}$ & $-0.4(-0.8$ to 0.1$)$ & $-1.2(-1.6 \text { to }-0.8)^{\star \star}$ & $0.7(0.3$ to 1.1$)$ & 0.002 \\
\hline $\mathrm{HbA} 1 \mathrm{C}, \%$ & $-0.4(-0.6 \text { to }-0.2)^{\star \star}$ & $-0.8(-1.0 \text { to }-0.5)^{\star \star}$ & $0.3(0.1$ to 0.4$)$ & 0.001 \\
\hline HOMA-IR & $-1.0(-1.7 \text { to }-0.3)^{\star}$ & $-4.4(-5.8 \text { to }-3.0)^{\star \star}$ & $2.0(1.2$ to 2.7$)$ & $<0.001$ \\
\hline \multicolumn{5}{|l|}{ Lipids } \\
\hline Total cholesterol, mmol/L & $-0.1(-0.2$ to 0.1$)$ & $-0.6(-0.8 \text { to }-0.4)^{\star \star}$ & $0.6(0.3$ to 0.8$)$ & $<0.001$ \\
\hline LDL cholesterol, mmol/L & $0.0(-0.2$ to 0.2$)$ & $-0.7(-0.8 \text { to }-0.5)^{\star \star}$ & 0.7 (0.5 to 0.9$)$ & $<0.001$ \\
\hline $\mathrm{HDL}$ cholesterol, $\mathrm{mmol} / \mathrm{L}$ & $0.1(0.1 \text { to } 0.2)^{\star \star}$ & $0.3(0.3 \text { to } 0.4)^{\star \star}$ & $0.2(0.1$ to 0.3$)$ & $<0.001$ \\
\hline Triglycerides, mmol/L & $-0.3(-0.5 \text { to }-0.2)^{\star \star}$ & $-0.7(-0.8 \text { to }-0.5)^{\star \star}$ & $0.3(0.2$ to 0.5$)$ & $<0.001$ \\
\hline
\end{tabular}

${ }^{\star} P<0.05{ }^{* \star} P<0.001$.

${ }^{a}$ Adjusted within-group values. Mean $(95 \% \mathrm{Cl})$. Within-group changes of the various outcome variables (left column) were analyzed with paired-samples $t$-test. Analyses of covariance (ANCOVA) including age, gender, history of coronary artery disease, baseline BMI, MAP, heart rate, and baseline status in dependent variable as covariates for ${ }_{\text {cf }} \mathrm{PWV}$, age, gender, history of coronary artery disease baseline BMI, and baseline status in dependent variable as covariates for systolic blood pressure, diastolic blood pressure and pulse pressure. Age, gender, and baseline status in dependent variable was included as covariates in ANCOVA analysis of heart rate, anthropometric measures, glucose metabolism, and lipids.

${ }_{\text {cf }} \mathrm{PWV}$ than controls completing an ILI-program. In addition, and to some surprise, despite considerable 1-year weight losses in both treatment groups $(10 \%$ and $32 \%)$, the average ${ }_{\mathrm{cf}} \mathrm{PWV}$ did not change significantly within either group. To our knowledge, this is the first study to compare the effect of bariatric surgery and an ILI-program on ${ }_{\text {cf }} \mathrm{PWV}$. Previous studies, including our own, have demonstrated the beneficial short-term (7 weeks-6 months) effects of various lifestyle intervention programs on ${ }_{\mathrm{cf}} \mathrm{PWV}$ in normal weight (15), overweight $(16)$, and obese populations $(17,18)$. In contrast, our results did not confirm the findings of some previous prospective long-term (1 year) studies demonstrating significant reductions of aortic stiffness after weight loss induced by diet alone (19), diet and physical activity (20), diet or bariatric surgery (21), and diet with/without orlistat (22). Rather, and to the contrary, the adjusted ${ }_{\mathrm{cf}} \mathrm{PWV}$ remained stable in both the GBS- and the ILI-group 1 year after start of treatment.

The apparent discrepancy between previous studies and the present one might have several explanations. First, in contrast with our study of treatment seeking morbidly obese subjects, several previous studies (19-21) included obese but otherwise healthy populations. It is plausible that subjects with obesity related comorbid- ities, like the majority of our patients, have a higher frequency of structural changes associated with aortic stiffness in the aortic wall than their metabolically healthy obese counterparts $(9,10)$ and that the potential reversal of such changes may take several years (8). Further, one previous study included nontreatment seeking diabetic, but otherwise healthy obese individuals (22). Approximately two-thirds of the participants achieved a decrease in ${ }_{\text {ff }} \mathrm{PWV}$ after 1 year, and individuals with the highest baseline values showed the largest improvement. This finding might partly be explained by regression to the mean, and the lack of adjustments for possible confounding factors could partly explain the difference in results compared to our own. Additionally, a significant number of participants in the GBS-group stopped taking antihypertensive and antidiabetic drugs during the study. The former might partly explain the modest decrease in blood pressure and, as cfPWV is partly pressure dependent, the absence of beneficial changes in aortic stiffness in the surgical group. In fact, antihypertensive drugs may reduce aortic stiffness beyond their blood pressure lowering effects (32). In addition, the use of metformin (33) and statins (34) have been associated with reduced arterial stiffness. The discontinuation of metformin might also have worked against any weight loss induced decline in ${ }_{\mathrm{cf}} \mathrm{PWV}$. 

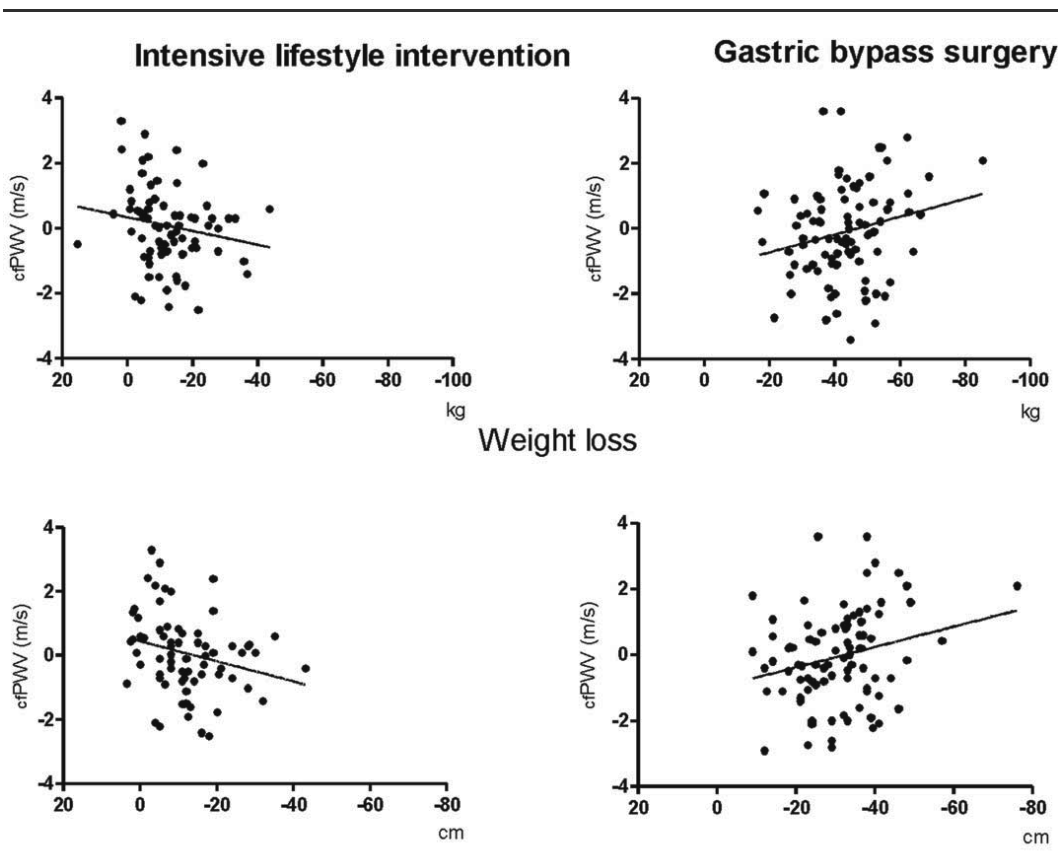

Waist circumference
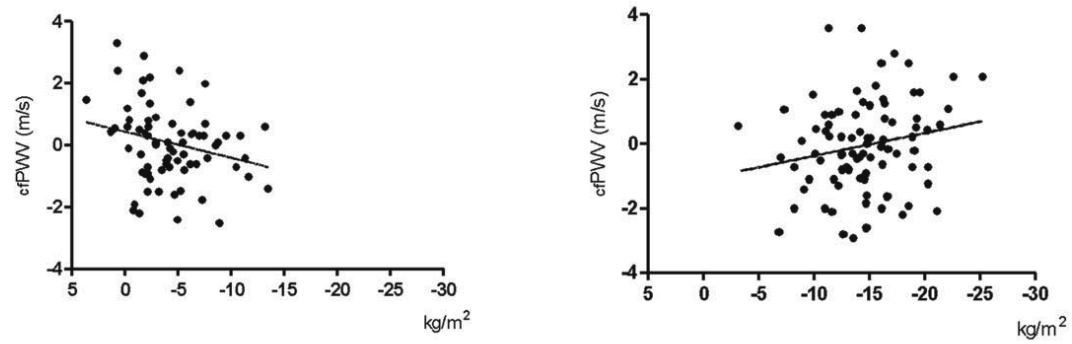

Body Mass Index

Figure 4 Scatter plots of the relationship between changes in the explanatory variables body weight, BMl and waist circumference, and changes in the effect variable ${ }_{\mathrm{cP}} \mathrm{PWV}$ within each treatment group.

Importantly, aortic stiffness is influenced by both functional and structural factors $(9,10,35,36)$. The results from the present analysis demonstrate that the short-term reduction of ${ }_{\mathrm{cf}} \mathrm{PWV}$ observed after 7 weeks of ILI (18) was a temporary one, and thus likely to be mediated by changes in functional factors like increased physical fitness, decreased heart rate and blood pressure and decreased activity in the sympathetic nervous system $(9,10)$. The findings that the greatest reductions in heart rate and blood pressure as well as ${ }_{\mathrm{cf}} \mathrm{PWV}$ throughout the study period were observed in the ILI-group after 7 weeks of follow-up also support this. Accordingly, the small longterm reductions in blood pressure and heart rate might partly explain the lack of association between treatment choice and changes in ${ }_{\text {cf }} \mathrm{PWV}$ as shown in the present study.

Post hoc subgroup analyses did not reveal any significant association between weight loss and changes in ${ }_{\mathrm{cf}} \mathrm{PWV}$ after lifestyle intervention, which is in accordance with the negative findings from the SAVE trial (37). The apparent detrimental effect of surgically induced weight loss on arterial stiffness was somewhat surprising and difficult to explain. Importantly, given the well known limitations of post hoc subgroup analyses, this finding should be interpreted with caution (38). One might, however, speculate that a 
massive weight loss may lead to a reflex increase in arterial resistance as the hyporesponsive baroreflex caused by obesity related chronic vasodilatation is abolished (39).

It is unlikely that our results are biased by measurement error since all measurements were conducted at one center by two trained operators (EG and $\mathrm{NN}$ ), both of whom were present at all measurements. Our findings are further strengthened by the relatively large sample size, long follow-up time and a considerable weight loss following both interventions. In addition, all of the physical exercise sessions in ILI were supervised by qualified personnel.

The major limitation of the study is the nonrandomized design. Since both treatments are publicly funded and patients are supposed to take an active role in a decision over treatment choice (40), we found it ethically most appropriate to perform a nonrandomized comparative study. Other minor limitations include the lack of data describing patients' adherence to the prescribed interventions (diet and physical activity) and the use of self-reported use of medications and level of physical activity. In addition, the use of sequential measurements at the carotid and femoral sites might have affected the reliability of the ${ }_{\mathrm{cf}} \mathrm{PWV}$ measurements. However, to minimize this potential bias ${ }_{\text {cf }} \mathrm{PWV}$ was measured three times at each measurement point, and the averages of these measurements were used in the calculations. Finally, we cannot exclude the possibility of a false negative result (type 2-error), but a post-hoc power analysis based on the number of participants completing the study demonstrated a power of more than $80 \%$ to detect a clinically meaningful between group difference in ${ }_{c f} \mathrm{PWV}$ change of $0.6 \mathrm{~m} / \mathrm{s}(\alpha=0.05)$.

\section{Perspective}

Aortic stiffness measured by ${ }_{c f} \mathrm{PWV}$ is not only a measure of agerelated aortic stiffening (10), but also a potential risk factor for cardiovascular disease (7). The findings of the present study suggest that long-term aortic stiffness in morbidly obese individuals is not significantly influenced by weight loss following bariatric surgery or an intensive lifestyle program. Our findings need further verification in future studies. $\mathrm{O}$

\section{Acknowledgments}

EG and NN conducted all measurements. EG, JH, and NN analyzed data. All authors were involved in writing the paper and had final approval of the submitted and published versions. We thank the patients who took part in this study. Further, we thank the following employees at the Morbid Obesity Center at Vestfold Hospital Trust: Dr.med Erling Saltvedt, Heidi Omre Fon, Berit Mossing Bjørkås, and Linda Mathisen for their assistance with sampling and logistics, $\mathrm{PhD}$. Harald Weedon-Fekjær, Institute of Basic Medical Sciences at University of Oslo, for statistical support, and Matthew McGee for proofreading the manuscript.

\section{(C) 2014 The Obesity Society}

\section{References}

1. Finucane MM, Stevens GA, Cowan MJ, et al. National, regional, and global trends in body-mass index since 1980: Systematic analysis of health examination surveys and epidemiological studies with 960 country-years and 9.1 million participants. Lancet 2011;377:557-567.

2. Must A, Spadano J, Coakley EH, Field AE, Colditz G,Dietz WH. The disease burden associated with overweight and obesity. JAMA 1999;282:1523-1529.
3. Rider OJ, Francis JM, Ali MK, et al. Beneficial cardiovascular effects of bariatric surgical and dietary weight loss in obesity. J Am Coll Cardiol 2009;54:718-726.

4. McAuley PA, Blair SN. Obesity paradoxes. J Sports Sci 2011;29:773-782.

5. Anderson KM, Wilson PW, Odell PM, Kannel WB. An updated coronary risk profile. A statement for health professionals. Circulation 1991;83:356-362.

6. Ben-Shlomo Y, Spears M, Boustred C, et al. Aortic pulse wave velocity improves cardiovascular event prediction: An individual participant metaanalysis of prospective observational data from 17,635 subjects. J Am Coll Cardiol 2014;63:636-646.

7. Vlachopoulos C, Aznaouridis K, Stefanadis C. Prediction of cardiovascular events and all-cause mortality with arterial stiffness: A systematic review and metaanalysis. J Am Coll Cardiol 2010;55:1318-1327.

8. Safar ME, Laurent S, Pannier BM, London GM. Structural and functional modifications of peripheral large arteries in hypertensive patients. J Clin Hypertens 1987;3:360-367.

9. Tanaka H, Safar ME. Influence of lifestyle modification on arterial stiffness and wave reflections. Am J Hypertens 2005;18:137-144.

10. Lakatta EG. Arterial and cardiac aging: Major shareholders in cardiovascular disease enterprises: Part III: Cellular and molecular clues to heart and arterial aging. Circulation 2003;107:490-497.

11. Laurent S, Boutouyrie P, Asmar R, et al. Aortic stiffness is an independent predictor of all-cause and cardiovascular mortality in hypertensive patients. Hypertension 2001;37:1236-1241.

12. Willum-Hansen T, Staessen JA, Torp-Pedersen C, et al. Prognostic value of aortic pulse wave velocity as index of arterial stiffness in the general population. Circulation 2006;113:664-670.

13. Laurent S, Cockcroft J, Van Bortel L, et al. Expert consensus document on arterial stiffness: Methodological issues and clinical applications. Eur Heart $J$ 2006;27: 2588-2605.

14. Mansia G, De Backer G, Dominiczak A, et al. 2007 ESH-ESC Guidelines for the management of arterial hypertension: The task force for the management of arterial hypertension of the European Society of Hypertension (ESH) and of the European Society of Cardiology (ESC). Blood Press 2007;16:135-232.

15. Yoshizawa M, Maeda S, Miyaki A, et al. Effect of 12 weeks of moderate-intensity resistance training on arterial stiffness: A randomised controlled trial in women aged 32-59 years. Br J Sports Med 2009;43:615-618.

16. Guimaraes GV, Ciolac EG, Carvalho VO, D'Avila VM, Bortolotto LA, Bocchi EA. Effects of continuous vs. interval exercise training on blood pressure and arterial stiffness in treated hypertension. Hypertens Res 2010;33:627-632.

17. Dengo AL, Dennis EA, Orr JS, et al. Arterial destiffening with weight loss in overweight and obese middle-aged and older adults. Hypertension 2010;55:855861 .

18. Nordstrand N, Gjevestad E, Hertel JK, et al. Arterial stiffness, lifestyle intervention and a low-calorie diet in morbidly obese patients-A nonrandomized clinical trial. Obesity (Silver Spring, Md) 2013;21:690-697.

19. Wycherley TP, Brinkworth GD, Keogh JB, Noakes M, Buckley JD, Clifton PM. Long-term effects of weight loss with a very low carbohydrate and low fat diet on vascular function in overweight and obese patients. J Intern Med 2010;267:452 461.

20. Cooper JN, Buchanich JM, Youk A, et al. Reductions in arterial stiffness with weight loss in overweight and obese young adults: Potential mechanisms. Atherosclerosis 2012;223:485-490.

21. Rider OJ, Tayal U, Francis JM, et al. The effect of obesity and weight loss on aortic pulse wave velocity as assessed by magnetic resonance imaging. Obesity (Silver Spring, Md) 2010;18: 2311-2316.

22. Barinas-Mitchell E, Kuller LH, Sutton-Tyrrell K, et al. Effect of weight loss and nutritional intervention on arterial stiffness in type 2 diabetes. Diabetes Care 2006; 29:2218-2222.

23. Nordstrand N, Gjevestad E, Dinh KN, et al. The relationship between various measures of obesity and arterial stiffness in morbidly obese patients. BMC Cardiovasc Disord 2011;1:7.

24. World Medical Association declaration of Helsinki. Recommendations guiding physicians in biomedical research involving human subjects. JAMA 1997;277:925926.

25. Schofield WN. Predicting basal metabolic rate, new standards and review of previous work. Hum Nutr Clin Nutr 1985;39(Suppl 1):5-41.

26. Wing RR, Hill JO. Successful weight loss maintenance. Annu Rev Nutr 2001;21: 323-341.

27. Wildman RP, Mackey RH, Bostom A, Thompson T, Sutton-Tyrrell K. Measures of obesity are associated with vascular stiffness in young and older adults. Hypertension 2003;42:468-473.

28. Diagnosis and classification of diabetes mellitus. Diabetes Care 2008;31(Suppl 1): S55-S60.

29. Matthews DR, Hosker JP, Rudenski AS, Naylor BA, Treacher DF, Turner RC. Homeostasis model assessment: Insulin resistance and beta-cell function from fasting plasma glucose and insulin concentrations in man. Diabtologia 1985;28:412419. 
30. Friedewald WT, Levy RI, Fredrickson DS. Estimation of the concentration of low density lipoprotein cholesterol in plasma, without use of the preparative ultracentrifuge. Clin Chem 1972;18:499-502.

31. Naess O, Sogaard AJ, Arnesen E, et al. Cohort profile: Cohort of Norway (CONOR). Int J Epidemiol 2008;37:481-485.

32. Seidlerova J, Filipovsky J, Mayer O, Wohlfahrt P, Cifkova R. Positive effects of antihypertensive treatment on aortic stiffness in the general population. Hypertens Res 2014;37:64-68.

33. Agarwal N, Rice SP, Bolusani H, et al. Metformin reduces arterial stiffness and improves endothelial function in young women with polycystic ovary syndrome: A randomized, placebo-controlled, crossover trial. J Clin Endocrinol Metab 2010;95: 722-730.

34. Rizos EC, Agouridis AP, Elisaf MS. The effect of statin therapy on arterial stiffness by measuring pulse wave velocity: A systematic review. Curr Vasc Pharmacol 2010;8:638-644.
35. Hirsch J, Leibel RL, Mackintosh R, Aguirre A. Heart rate variability as a measure of autonomic function during weight change in humans. Am J Physiol 1991;261: R1418-R1423.

36. Arone LJ, Mackintosh R, Rosenbaum M, Leibel RL, Hirsch J. Autonomic nervous system activity in weight gain and weight loss. Am J Physiol 1995;269:R222-R225.

37. Hughes TM, Althouse AD, Niemczyk NA, Hawkins MS, Kuipers AL, SuttonTyrrell K. Effects of weight loss and insulin reduction on arterial stiffness in the SAVE trial. Cardiovasc Diabetol 2012;11:114.

38. Wang R, Lagakos SW, Ware JH, Hunter DJ, Drazen JM. Statistics in medicinereporting of subgroup analyses in clinical trials. N Engl J Med 2007;357:2189-2194.

39. Emdin M, Gastaldelli A, Muscelli E, et al. Hyperinsulinemia and autonomic nervous system dysfunction in obesity: Effects of weight loss. Circulation 2001; 103:513-519.

40. Jakobsen GS, Hofso D, Roislien J, Sandbu R, Hjelmesaeth J. Morbidly obese patients-who undergoes bariatric surgery? Obes Surg 2010;20:1142-1148. 
Supp Table 1 Self-reported physical activity at 59 weeks follow up in morbidly obese patients which completed the interventions.

\begin{tabular}{|c|c|c|c|c|}
\hline & & $\begin{array}{c}\text { Intensive lifestyle } \\
\text { intervention } \\
(n=60)\end{array}$ & $\begin{array}{l}\text { Gastric bypass } \\
\text { surgery } \\
(n=54)\end{array}$ & $P$-value \\
\hline \multirow[t]{4}{*}{ Light physical activity } & Nothing & $0(0)$ & $0(0)$ & 0.880 \\
\hline & $\leq 1$ hour/week & $1(2)$ & $1(2)$ & \\
\hline & 1-2 hours/week & $7(12)$ & $8(15)$ & \\
\hline & $\geq 3$ hours/week & $52(87)$ & $45(83)$ & \\
\hline \multirow{4}{*}{$\begin{array}{l}\text { Vigorous physical } \\
\text { activity }\end{array}$} & Nothing & $2(3)$ & $6(11)$ & 0.267 \\
\hline & $\leq 1$ hour/week & $5(8)$ & $7(13)$ & \\
\hline & 1-2 hours/week & $21(34)$ & $17(32)$ & \\
\hline & $\geq 3$ hours/week & $34(55)$ & $24(44)$ & \\
\hline \multirow[t]{4}{*}{ Work } & Sedentary work & $23(39)$ & $19(36)$ & 0.485 \\
\hline & Walking & $24(41)$ & $19(36)$ & \\
\hline & Walking and carrying & $11(19)$ & $11(21)$ & \\
\hline & Heavy manual labor & $1(2)$ & $4(8)$ & \\
\hline
\end{tabular}

Number (\%), between group differences analyzed using Chi-Square test. The response rate of self reported physical activity level was $78 \%$ in the ILI-group and $66 \%$ in the GBS group. 